\title{
EULER'S CONSTANT: EULER'S WORK AND MODERN DEVELOPMENTS
}

\author{
JEFFREY C. LAGARIAS
}

Abstract. This paper has two parts. The first part surveys Euler's work on the constant $\gamma=0.57721 \cdots$ bearing his name, together with some of his related work on the gamma function, values of the zeta function, and divergent series. The second part describes various mathematical developments involving Euler's constant, as well as another constant, the Euler-Gompertz constant. These developments include connections with arithmetic functions and the Riemann hypothesis, and with sieve methods, random permutations, and random matrix products. It also includes recent results on Diophantine approximation and transcendence related to Euler's constant.

\section{Contents}

1. Introduction

2. Euler's work

2.1. Background

2.2. Harmonic series and Euler's constant

2.3. The gamma function

2.4. Zeta values

2.5. Summing divergent series: the Euler-Gompertz constant

2.6. Euler-Mascheroni constant; Euler's approach to research

3. Mathematical developments

3.1. Euler's constant and the gamma function 557

3.2. Euler's constant and the zeta function

3.3. Euler's constant and prime numbers

3.4. Euler's constant and arithmetic functions

3.5. Euler's constant and sieve methods: the Dickman function

3.6. Euler's constant and sieve methods: the Buchstab function

3.7. Euler's constant and the Riemann hypothesis

3.8. Generalized Euler constants and the Riemann hypothesis 575

3.9. Euler's constant and extreme values of $\zeta(1+i t)$ and $L\left(1, \chi_{-d}\right)$

3.10. Euler's constant and random permutations: cycle structure 582

3.11. Euler's constant and random permutations: shortest cycle $\quad 586$

3.12. Euler's constant and random finite functions $\quad 589$

3.13. Euler's constant as a Lyapunov exponent 590

3.14. Euler's constant and periods

Received by the editors July 1, 2010, and, in revised form, December 24, 2012.

2010 Mathematics Subject Classification. Primary 11J02; Secondary 01A50, 11J72, 11J81, $11 \mathrm{M} 06$.

The research of the author was supported by NSF Grants DMS-0801029 and DMS-1101373. 
3.15. Diophantine approximations to Euler's constant

3.16. Transcendence results related to Euler's constant

4. Concluding remarks

Acknowledgments

About the Author

613

References

\section{INTRODUCTION}

Euler discovered the constant $\gamma$, defined by

$$
\gamma:=\lim _{n \rightarrow \infty}\left(\sum_{j=1}^{n} \frac{1}{j}-\log n\right) .
$$

This is a fundamental constant, now called Euler's constant. We give it to 50 decimal places as

$$
\gamma=0.57721566490153286060651209008240243104215933593992 \cdots \text {. }
$$

In this paper we shall describe Euler's work on it, and its connection with values of the gamma function and Riemann zeta function. We consider as well its close relatives $e^{\gamma}=1.7810724 \cdots$ and $e^{-\gamma}=0.561459 \cdots$. We also inquire as to its possible meaning by describing many situations in mathematics where it occurs.

The constant $\gamma$ is often known as the Euler-Mascheroni constant, after later work of Mascheroni discussed in Section 2.6. As explained there, based on their relative contributions, it seems appropriate to name it after Euler.

There are many famous unsolved problems about the nature of this constant. The most well known one is:

Conjecture 1.0.1. Euler's constant is irrational.

This is a long-standing open problem. A recent and stronger version of it is the following.

Conjecture 1.0.2. Euler's constant is not a Kontsevich-Zagier period. In particular, Euler's constant is transcendental.

A period is defined by Kontsevich and Zagier [186] to be a complex constant whose real and imaginary parts separately are given as a finite sum of absolutely convergent integrals of rational functions in any number of variables with rational coefficients, integrated over a domain cut out by a finite set of polynomial equalities and inequalities with rational coefficients; see Section 3.14. Many constants are known to be periods, in particular all zeta values $\{\zeta(n): n \geq 2\}$. The set of all periods forms a ring $\mathcal{P}$ which includes the field $\overline{\mathbb{Q}}$ of all algebraic numbers. It follows that if Euler's constant is not a period, then it must be a transcendental number. Conjecture 1.0.2 also implies that $\gamma$ would be $\overline{\mathbb{Q}}$-linearly independent of all odd zeta values $\zeta(3), \zeta(5), \zeta(7), \ldots$ and of $\pi$.

This paper also presents results on another constant defined by Euler, the EulerGompertz constant

$$
\delta:=\int_{0}^{1} \frac{d v}{1-\log v}=\int_{0}^{\infty} \frac{e^{-t}}{1+t} d t=0.596347362323194 \cdots
$$


Here we adopt the notation of Aptekarev [13] for this constant (in which $\delta$ follows $\gamma$ ) and refer to it by the name given by Finch [116, Section 6.2], who denotes it $C_{2}$. Some results about $\delta$ intertwine directly with Euler's constant; see Section 3.5, Section 3.6. Section 3.15, and Section 3.16.

Euler's name is also associated with other constants such as $e=2.71828 \cdots$ given by

$$
e:=\sum_{n=0}^{\infty} \frac{1}{n !}
$$

He did not discover this constant, but did standardize the symbol " $e$ " for its use. He used the notation $e$ in his 1737 essay on continued fractions ([97, p. 120], cf. [98]), where he determined its continued fraction expansion

$$
e=[2 ; 1,2,1,1,4,1,1,6,1,1,8, \ldots]=2+\frac{1}{1+\frac{1}{2+\frac{1}{1+\frac{1}{1+\cdots}}}},
$$

from which he deduced that it was irrational. Elsewhere he found the famous relation $e^{\pi i}=-1$. The constant $e$ is also conjectured not to be a KontsevichZagier period. It is well known to be a transcendental number, consistent with this conjecture.

In this paper we review Euler's work on the constant $\gamma$ and on mathematical topics connected to it and survey subsequent developments. It is correspondingly divided into two parts, which may be read independently. We have also aimed to make individual subsections readable out of order.

The first part of this paper considers the work of Euler. Its emphasis is historical, and it retains some of the original notation of Euler. It is a tour of part of Euler's work related to zeta values and other constants and methods of finding relations between them. Euler did extensive work related to his constant: he returned to its study many times. The basic fact to take away is that Euler did an enormous amount of work directly on his constant and related areas, far more than is commonly appreciated.

The second part of this paper addresses mathematical developments made since Euler's time concerning Euler's constant. Since Euler's constant is an unusual constant that seems unrelated to other known constants, its appearance in different mathematical subjects can be a signal of possible connections between these subjects. We present many contexts in which Euler's constant appears. These include connections to the Riemann hypothesis, to random matrix theory, to probability theory, and to other subjects. There is a set of strong analogies between factorizations of random integers in the interval $[1, n]$ and cycle structures of random permutations in the symmetric group $S_{N}$, taking $N$ to be proportional to $\log n$. In this analogy there appears another constant that might have appealed to Euler: the Golomb-Dickman constant, which can be defined as

$$
\lambda:=\int_{0}^{1} e^{L i(x)} d x=0.624329988543550 \cdots,
$$


where $\operatorname{Li}(x):=\int_{0}^{x} \frac{d t}{\log t}$; see Section 3.10 The constant $\lambda$ appears in connection with the distribution of the longest cycle of a random permutation, while the constant $e^{-\gamma}$ appears in connection with the distribution of the shortest cycle. The discovery of this analogy is described at the beginning of Section 3.11. It was found by Knuth and Trabb-Pardo [180, through noticing a numerical coincidence to ten decimal places between two computed constants, both subsequently identified with the Golomb-Dickman constant.

In passing we present many striking and elegant formulas, which exert a certain fascination in themselves. Some of the formulas presented were obtained by specialization of more general results in the literature. Other works presenting striking formulas include the popular book of J. Havil [158, which is devoted extensively to mathematics around Euler's constant, and Finch [116, Sects. 1.5 and 4.5].

In Section 4 we make concluding remarks and briefly describe other directions of work related to Euler's constant.

\section{EULER's WORK}

Euler (1707-1783) was born in Basel, and entered the University of Basel at age 13 to study theology. He also had private tutorials in mathematics from Johann Bernoulli (1667-1748), who was Professor of Mathematics at the University of Basel. Johann recognized Euler's mathematical gift and convinced his family to let him switch to mathematics. Euler was excellent at computation and had an essentially photographic memory. He had great persistence and returned over and over again to problems that challenged him.

Here we only scratch the surface of Euler's enormous output, which contains over 800 works. These include more than 20 books, some surveyed in Gautschi [125]. We trace a thread comprising results corresponding to one of his intereststhe determination of specific constants given by infinite sums, definite integrals, infinite products, and divergent series, and finding identities giving interrelations among these constants. Ancillary to this topic is the related problem of determining numerical values of these constants; computing such values allows one to guess and test possible identities.

In his work Euler made extensive calculations, deriving approximations of numbers to many decimal places. He often reported results of calculations obtained using various approximation schemes and sometimes compared the values he obtained by the different methods. In Euler's numerical results reported below the symbol $\approx$ is used to mean "the result of an approximate calculation". The symbol $\approx$ does not imply agreement to the number of digits provided. An underline of a digit in such an expansion indicates the first place where it disagrees with the known decimal expansion.

We refer to Euler's papers by their index numbers in the Enëstrom catalogue (86]). These papers may be found online in the Euler archive (88]). For discussions of Euler's early work up to 1750, see Sandifer [266], [267]. For his work on the Euler(-Maclaurin) summation formula, see Hofmann [167] and Knoebel et al. [178, Chapter 1]. For other discussions of his work on the zeta function, see Stäckel [285], Ayoub [21, Weil [315, Chapter 3], and Varadarajan [310, Chapter 3]. For Euler's life and work see Calinger [54] and other articles in Bradley and Sandifer [41. 
2.1. Background. Euler spent much effort evaluating the sums of reciprocal powers

$$
\zeta(m):=\sum_{j=1}^{\infty} \frac{1}{j^{m}}
$$

for integer $m \geq 2$. He obtained the formula $\zeta(2)=\frac{\pi^{2}}{6}$, solving the "Basel problem", as well as formulas for all even zeta values $\zeta(2 n)$. He repeatedly tried to find analogous formulas for the odd zeta values $\zeta(2 n+1)$ which would explain the nature of these numbers. Here he did not completely succeed, and the nature of the odd zeta values remains unresolved to this day. Euler's constant naturally arises in this context, in attempting to assign a value to $\zeta(1)$.

Euler discovered the constant $\gamma$ in a study of the harmonic numbers

$$
H_{n}:=\sum_{j=1}^{n} \frac{1}{j},
$$

which are the partial sums of the harmonic series. The series " $\zeta(1)$ " $=\sum_{n=1}^{\infty} \frac{1}{n}$ diverges, and Euler approached it by (successfully) finding a function $f(z)$ (of a real or complex variable $z$ ) that interpolates the harmonic number values, i.e. it has $f(n)=H_{n}$ for integer $n \geq 1$. The resulting function, denoted $H_{z}$ below, is related to the gamma function via (2.2.2). In studying it he also studied, for integer $m$, the related sums

$$
H_{n, m}:=\sum_{j=1}^{n} \frac{1}{j^{m}},
$$

which we will term m-harmonic numbers; in this notation $H_{n, 1}=H_{n}$.

Euler obtained many interrelated results on his constant $\gamma$. These are intertwined with his work on the gamma function and on values of the (Riemann) zeta function. In Sections 2.2-2.4 we present results that he obtained for Euler's constant, for the gamma function and its logarithmic derivative, and for values of the zeta function at integer arguments, concentrating on explicit formulas. In Section 2.5 we discuss a paper of Euler on how to sum divergent series, applied to the example $0 !-1 !+$ $2 !-3 !+\cdots$. His analysis produced a new constant, the Euler-Gompertz constant $\delta$, given above in (1.1). In Section 2.6 we review the history of the name EulerMascheroni constant, the adoption of the notation $\gamma$ for it, and summarize Euler's approach to research.

Taken together Euler's many papers on his constant $\gamma$, on the related values $\zeta(n)$ for $n \geq 2$, and other values show his great interest in individual constants ("notable numbers") and on their arithmetic interrelations.

2.2. Harmonic series and Euler's constant. One strand of Euler's early work involved finding continuous interpolations of various discrete sequences. For the sequence of factorials this led to the gamma function, discussed below. In the 1729 paper [90, E20] he notes that the harmonic numbers $H_{n}=\sum_{k=1}^{n} \frac{1}{k}$ are given by the integrals

$$
H_{n}:=\int_{0}^{1} \frac{1-x^{n}}{1-x} d x
$$


He proposes a function interpolating $H_{n}$ by treating $n$ as a continuous variable in this integral. The resulting interpolating function, valid for real $z \geq 0$, is

$$
H_{z}:=\int_{0}^{1} \frac{1-x^{z}}{1-x} d x
$$

Using this definition, he derives the formula

$$
H_{\frac{1}{2}}=2-2 \log 2 .
$$

The function $H_{z}$ is related to the digamma function $\psi(z)=\frac{d}{d z} \log \Gamma(z)$ by

$$
H_{z}=\psi(z+1)+\gamma
$$

see Section 2.3 and also Theorem 3.1 .1

In a paper written in 1731 (On harmonic progressions) Euler [93, E43] summed the harmonic series in terms of zeta values as follows, and he computed Euler's constant to five decimal places.

Theorem 2.2.1 (Euler 1731). The limit

$$
\gamma=\lim _{n \rightarrow \infty}\left(H_{n}-\log n\right)
$$

exists. It is given by the (conditionally) convergent series

$$
\gamma=\sum_{n=2}^{\infty}(-1)^{n} \frac{\zeta(n)}{n} .
$$

Euler explicitly observes that this series (2.2.3) converges (conditionally) since it is an alternating series with decreasing terms. He reports that the constant $\gamma \approx 0.57721 \underline{8}$.

Euler obtains the formula (2.2.3) using the expansion

$$
\log (1+x)=\sum_{k=1}^{\infty}(-1)^{k+1} \frac{x^{k}}{k}
$$

which was found by Nicholas Mercator (ca. 1620-1687) 218 in 1668. Evaluating this formula at $x=1, \frac{1}{2}, \ldots, \frac{1}{n}$ Euler observes the following in tabular form:

$$
\begin{aligned}
\log 2 & =1-\frac{1}{2} \cdot\left(\frac{1}{1}\right)^{2}+\frac{1}{3} \cdot\left(\frac{1}{1}\right)^{3}-\cdots, \\
\log \frac{3}{2} & =\frac{1}{2}-\frac{1}{2} \cdot\left(\frac{1}{2}\right)^{2}+\frac{1}{3} \cdot\left(\frac{1}{2}\right)^{3}+\cdots, \\
\log \frac{4}{3} & =\frac{1}{3}-\frac{1}{2} \cdot\left(\frac{1}{3}\right)^{2}+\frac{1}{3} \cdot\left(\frac{1}{3}\right)^{3}+\cdots, \\
\log \frac{5}{4} & =\frac{1}{4}-\frac{1}{2} \cdot\left(\frac{1}{4}\right)^{2}+\frac{1}{4} \cdot\left(\frac{1}{4}\right)^{3}+\cdots .
\end{aligned}
$$

Summing by columns the first $n$ terms, he obtains

$$
\log (n+1)=H_{n}-\frac{1}{2} H_{n, 2}+\frac{1}{3} H_{n, 3}-\cdots .
$$

One may rewrite this as

Now one observes that for $j \geq 2$,

$$
H_{n}-\log (n+1)=\frac{1}{2} H_{n, 2}-\frac{1}{3} H_{n, 3}+\cdots .
$$

$$
\lim _{n \rightarrow \infty} H_{n, j}=H_{\infty, j}=\zeta(j) .
$$


Taking this limit as $n \rightarrow \infty$ term by term in (2.2.4) formally gives the result (2.2.3).

We note that such representations of infinite sums in a square array, to be summed in two directions, were already used repeatedly by Johann Bernoulli's older brother Jacob Bernoulli (1654-1705) in his 1689 book on summing infinite series ([29]). Another nice example of an identity found by array summation is

$$
(\zeta(2)-1)+(\zeta(3)-1)+(\zeta(4)-1)+\cdots=1,
$$

using

$$
\begin{aligned}
\zeta(2)-1 & =\frac{1}{2^{2}}+\frac{1}{3^{2}}+\frac{1}{4^{2}}+\frac{1}{5^{2}}+\cdots \\
\zeta(3)-1 & =\frac{1}{2^{3}}+\frac{1}{3^{3}}+\frac{1}{4^{3}}+\frac{1}{5^{3}}+\cdots \\
\zeta(4)-1 & =\frac{1}{2^{4}}+\frac{1}{3^{4}}+\frac{1}{4^{4}}+\frac{1}{5^{4}}+\cdots
\end{aligned}
$$

since the column sums yield the telescoping series

$$
\frac{1}{2^{2}} \times 2+\frac{1}{3^{2}} \times \frac{3}{2}+\frac{1}{4^{2}} \times \frac{4}{3}+\cdots=\frac{1}{1 \cdot 2}+\frac{1}{2 \cdot 3}+\frac{1}{3 \cdot 4}+\cdots=1 .
$$

Compare (2.2.5) to Euler's expression (2.2.11) below for Euler's constant.

In 1732 Euler [91, E25] stated his first form of his summation formula, without a proof. He then gives it in detail in his 1735 paper [94, E47], published 1741. Letting $s$ denote the sum of the function $t(n)$ over some set of integer values (say $n=1$ to $N$ ), he writes

$$
s=\int t d n+\alpha t+\beta \frac{d t}{d n}+\gamma \frac{d^{2} t}{d n^{2}}+\delta \frac{d^{3} t}{d n^{3}}+\text { etc. }
$$

where $\alpha=\frac{1}{2}, \beta=\frac{\alpha}{2}-\frac{1}{6}, \gamma=\frac{\beta}{2}-\frac{\alpha}{6}+\frac{1}{24}, \delta=\frac{\gamma}{2}-\frac{\beta}{6}+\frac{\alpha}{24}-\frac{1}{120}$, etc. These linear equations for the coefficients are solvable to give $\alpha=\frac{1}{2}, \beta=\frac{1}{12}, \gamma=0, \delta=-\frac{1}{720}$, etc. Thus

$$
s=\int t d n+\frac{t}{2}+\frac{1}{12} \frac{d t}{d n}-\frac{1}{720} \frac{d^{3} t}{d n^{3}}+\text { etc. }
$$

The terms on the right must be evaluated at both endpoints of this interval being summed. In modern terms,

$$
\begin{aligned}
\sum_{n=1}^{N} t(n)=\int_{0}^{N} t(n) d n & +\frac{1}{2}(t(N)-t(0))+\frac{B_{2}}{2 !}\left(\frac{d t(N)}{d n}-\frac{d t(0)}{d n}\right) \\
& -\frac{B_{4}}{4 !}\left(\frac{d^{3} t(N)}{d n^{3}}-\frac{d^{3} t(0)}{d n^{3}}\right)+\cdots,
\end{aligned}
$$

where the $B_{2 k}$ are Bernoulli numbers, defined in (2.2.6) below, and the sum must be cut off at a finite point with a remainder term (cf. [293, Section 1.0]). Euler's formulas omit any remainder term, and he applies them by cutting off the right side sum at a finite place, and then using the computed value of the truncated right side sum as an approximation to the sum on the left, in effect making the assumption that the associated remainder term is small.

A similar summation formula was obtained independently by Colin Maclaurin (1698-1746), who presented it in his 1742 two-volume work on Fluxions [208]. A comparison of their formulas is made by Mills [220, who observes they are substantially identical, and that neither author keeps track of the remainder term. 
It is appropriate that this formula, now with the remainder term included, is called the Euler-Maclaurin summation formula.

Jacob Bernoulli introduced the numbers now bearing his name (with no subscripts), in Ars Conjectandi [30, published posthumously in 1713. They arose in evaluating (in modern notation) the sums of $n$th powers

$$
\sum_{k=1}^{m-1} k^{n}=\frac{1}{n+1}\left(\sum_{k=0}^{n}\left(\begin{array}{c}
n+1 \\
k
\end{array}\right) B_{k} m^{n+1-k}\right) .
$$

We follow the convention that the Bernoulli numbers $B_{n}$ are those given by the expansion

$$
\frac{t}{e^{t}-1}=\sum_{n=0}^{\infty} \frac{B_{n}}{n !} t^{n}
$$

so that

$$
B_{1}=-\frac{1}{2}, B_{2}=\frac{1}{6}, B_{3}=0, B_{4}=-\frac{1}{30}, B_{5}=0, B_{6}=\frac{1}{42}, \ldots
$$

Euler later named the Bernoulli numbers in his 1768 paper (in 107, E393]) (On the sum of series involving the Bernoulli numbers), and there listed the first few as $\frac{1}{6}, \frac{1}{30}, \frac{1}{42}, \frac{1}{30}, \frac{5}{66}$. His definition led to the convention 1 that the notation $B_{n}$ corresponds to $\left|B_{2 n}\right|$ in (2.2.6). This convention is followed in the classic text of Whittaker and Watson [317, p. 125].

In his 1755 book on differential calculus [103, E212, Part II, Chapters 5 and 6] Euler presented his summation formula in detail, with many worked examples. (See Pengelley [244, 245] for a translation and for a detailed discussion of this work.) In Section 122 Euler computes the Bernoulli numbers $\left|B_{2 n}\right|$ through $n=15$. In Section 129 he remarks that:

The [Bernoulli numbers] form a highly divergent sequence, which grows more strongly than any geometric series of growing terms 2

In Section 142 he considers the series for the harmonic numbers $s=H_{x}=\sum_{n=1}^{x} \frac{1}{n}$, where his summation formula reads (in modern notation)

$$
s=\log x+\left(\frac{1}{2 x}-\frac{\left|B_{2}\right|}{2 x^{2}}+\frac{\left|B_{4}\right|}{4 x^{4}}-\frac{\left|B_{6}\right|}{6 x^{6}}+\cdots\right)+C,
$$

where the constant $C$ to be determined is Euler's constant. He remarks that this series is divergent. He substitutes $x=1$, stating that $s=1$ in (2.2.7), obtaining formally

$$
C=\frac{1}{2}+\frac{\left|B_{2}\right|}{2}-\frac{\left|B_{4}\right|}{4}+\frac{\left|B_{6}\right|}{6}-\frac{\left|B_{8}\right|}{8}+\cdots,
$$

although the right side diverges. Substituting this expression in (2.2.7) yields

$$
\begin{aligned}
s=\log x & +\left(\frac{1}{2 x}-\frac{\left|B_{2}\right|}{2 x^{2}}+\frac{\left|B_{4}\right|}{4 x^{4}}-\frac{\left|B_{6}\right|}{6 x^{6}}+\frac{\left|B_{8}\right|}{8 x^{8}}-\cdots\right) \\
& +\left(\frac{1}{2}+\frac{\left|B_{2}\right|}{2}-\frac{\left|B_{4}\right|}{4}+\frac{\left|B_{6}\right|}{6}-\frac{\left|B_{8}\right|}{8}+\cdots\right) .
\end{aligned}
$$

\footnotetext{
${ }^{1}$ However in [107] and elsewhere Euler does not use subscripts, writing $\mathfrak{A}, \mathfrak{B}, \mathfrak{C}$ etc.

2 "pariter ac Bernoulliani $\mathfrak{A}, \mathfrak{B}, \mathfrak{C}, \mathfrak{D} \& c$. serium maxime divergentem, quae etiam magis increscat, quam ulla series geometrica terminis crescentibus procedens." [Translation: David Pengelley 244, 245.]
} 
In Section 143 he sets $x=10$ in (2.2.7) so that $s=H_{10}$, and gives the numerical value $s=2.928968253968253968$. The right side contains $C$ as an unknown, and by evaluating the other terms (truncating at a suitable term) he finds

$$
C \approx 0.577215664901532 \underline{5},
$$

a result accurate to 15 places. We note that the divergent series on the right side of (2.2.7) is the asymptotic expansion of the digamma function $\psi(x+1)+\gamma$, where $\psi(x)=\frac{\Gamma^{\prime}(x)}{\Gamma(x)}$ (see Theorem 3.1.3) , and in effect Euler truncates the asymptotic expansion at a suitable term to obtain a good numerical approximation.

Over his lifetime Euler obtained many different numerical approximations to Euler's constant. He calculated approximations using truncated divergent series, and his papers report the value obtained, sometimes with a comment on accuracy, sometimes not. In contrast, some of the convergent series he obtained for Euler's constant converge slowly and proved of little value for numerics. He also explored various series acceleration methods, which he applied to both convergent and divergent series. He compared and cross-checked his work with various different numerical methods. In this way he obtained confidence as to how many digits of accuracy he had obtained using the various methods, and also obtained information on the reliability of his calculations using divergent series.

In 1765 in a paper studying the gamma function [106, E368] (which will be discussed in more detail in Section 2.3), he derives the formula $\Gamma^{\prime}(1)=-\gamma$. It is given in Section 14 of his paper as equation (2.3.6) in Section 2.3, which is equivalent to the integral formula

$$
-\gamma=\int_{0}^{\infty} e^{-x} \log x d x
$$

This result follows from differentiation under the integral sign of Euler's integral (2.3.4) in Section 2.3.

In a 1768 paper mainly devoted to relating Bernoulli numbers to values of $\zeta(n)$, Euler [107, E393] obtains more formulas for $\gamma$ (denoting it $O$ ). In Section 24 he reports the evaluation of $\gamma$ found earlier and remarks:

$O=0,5772156649015325$. This number seems also the more noteworthy because even though I have spent much effort in investigating it, I have not been able to reduce it to a known kind of quantity

He derives in Section 25 the integral formula

$$
\gamma=\int_{0}^{\infty}\left(\frac{e^{-y}}{1-e^{-y}}-\frac{e^{-y}}{y}\right) d y .
$$

Here we may note that the integrand

$$
\frac{e^{-y}}{1-e^{-y}}-\frac{e^{-y}}{y}=\frac{1}{y}\left(\frac{y}{e^{y}-1}-e^{-y}\right)=\sum_{n=1}^{\infty}\left(B_{n}-(-1)^{n}\right) \frac{y^{n-1}}{n !} .
$$

\footnotetext{
3 " $O=0.5772156649015325$ qui numerus eo maiori attentione dignus videtur, quod eum, cum olim in hac investigatione multum studii consumsissem, nullo modo ad cognitum quantitatum genus reducere valui." [Translation: Jordan Bell.]
} 
In Section 26 by change of variables he obtains

$$
\gamma=\int_{0}^{1}\left(\frac{1}{1-z}+\frac{1}{\log z}\right) d z
$$

In Section 27 he gives the following new formula for Euler's constant

$$
\gamma=\frac{1}{2}(\zeta(2)-1)+\frac{2}{3}(\zeta(3)-1)+\frac{3}{4}(\zeta(4)-1)+\cdots,
$$

and in Section 28 he obtains a formula in terms of $\log 2$ and the odd zeta values:

$$
\gamma=\frac{3}{4}-\frac{1}{2} \log 2+\sum_{k=1}^{\infty}\left(1-\frac{1}{2 k+1}\right)(\zeta(2 k+1)-1)
$$

He concludes in Section 29:

Therefore the question remains of great moment, of what character the number $O$ is and among what species of quantities it can be classified 4

In a 1776 paper [110, E583] (On a memorable number naturally occurring in the summation of the harmonic series) 5 Euler studied the constant $\gamma$ in its own right. This paper was not published until 1785, after his death. In Section 2 he speculates that the number $N=e^{\gamma}$ should be a notable number:

And therefore, if $x$ is taken to be an infinitely large number it will then be

$$
1+\frac{1}{2}+\frac{1}{3}+\cdots+\frac{1}{x}=C+\log x
$$

One may suspect from this that the number $C$ is the hyperbolic [= natural] logarithm of some notable number, which we put $=N$, so that $C=\log N$ and the sum of the infinite series is equal to the logarithm of the number $N \cdot x$. Thus it will be worthwhile to inquire into the value of this number $N$, which indeed it suffices to have defined to five or six decimal figures, since then one will be able to judge without difficulty whether this agrees with any known number or not 6

Euler evaluates $N=1.7810 \underline{6}$ and says he cannot connect it to any number he knows. He then gives several new formulae for $\gamma$, and tests their usefulness for calculating its value, comparing the values obtained numerically with the value 0.5772156649015325 that he previously obtained. His first new formula in Section 6 is

$$
1-\gamma=\frac{1}{2}(\zeta(2)-1)+\frac{1}{3}(\zeta(3)-1)+\frac{1}{4}(\zeta(4)-1)+\cdots .
$$

\footnotetext{
4 "Manet ergo quaestio magni momenti, cujusdam indolis sit numerus iste $O[:=\gamma]$ et ad quodnam genus quantitatum sit referendus." [Translation: Jordan Bell.]

${ }^{5}$ De numero memorabili in summatione progressionis harmonicae naturalis occurrente

6 "Quod si ergo numerus $x$ accipiatur magnus, tum erit

$$
1+\frac{1}{2}+\frac{1}{3}+\cdots+\frac{1}{z}=C+l x
$$

istum numerum $C[:=\gamma]$ esse logarithmum hyperbolicum cuiuspiam numeri notabilis, quem statuamus $=N$, ita ut sit $C=\log N$, et summa illius seriei infinitae aequeture logarithmo numeri $N x$, under operae pretium erit in valorem huius numeri $N$ inquirerre, quem quidem sufficiet ad quinque vel fex figuras decimales definiuisse, quoniam hinc non difficulter iduicari poterit, num cum quopiam numero cogito conueniat nec ne." [Translation: Jordan Bell.]
} 
Using the first 16 terms of this expansion, he finds $\gamma \approx 0,57721 \underline{6} 9$. He finds several other formulas, including, in Section 15, the formula

$$
1-\log \frac{3}{2}-\gamma=\frac{1}{3 \cdot 2^{2}}(\zeta(3)-1)+\frac{1}{5 \cdot 2^{4}}(\zeta(5)-1)+\frac{1}{7 \cdot 2^{6}}(\zeta(7)-1)+\cdots,
$$

from which he finds $\gamma \approx 0.577215664901 \underline{7913}$, a result accurate to 12 places. He concludes the paper with a list of eight formulas relating Euler's constant, $\log 2$, and even and odd zeta values. One striking formula (number VII), involving the odd zeta values is

$$
\gamma=\log 2-\sum_{k=1}^{\infty} \frac{\zeta(2 k+1)}{(2 k+1) 2^{2 k}} .
$$

In another 1776 paper [112, E629] (The expansion of the integral formula $\int \partial x\left(\frac{1}{1-x}+\frac{1}{\ell x}\right)$ with the term extended from $x=0$ to $x=1$ ), which was not published until 1789, Euler further investigated the integral formula (2.2.10) for his constant. He denotes its value by $n$, and says:

the number $n,[\ldots]$ which is found to be approximately

$$
0,5772156649015325 \text {, }
$$

whose value I have been able in no way to reduce to already known transcendental measures; therefore, it will hardly be useless to try to resolve the formula in many different ways 7

He then finds several expansions for related integrals. One of these starts from the identity, valid for $m, n \geq 0$,

$$
\int_{0}^{1} \frac{x^{m}-x^{n}}{\log x} d x=\log \left(\frac{m+1}{n+1}\right)
$$

and from this he deduces, for $n \geq 1$, that

$$
\int_{0}^{1} \frac{(1-x)^{n}}{\log x} d x=\log \left(\prod_{j=0}^{n}(j+1)^{(-1)^{j}\left(\begin{array}{c}
n \\
j
\end{array}\right)}\right) .
$$

This formula gives the moments of the measure $\frac{x}{\log (1-x)} d x$ on $[0,1]$.

2.3. The gamma function. Euler was not the originator of work on the factorial function. This function was initially studied by John Wallis (1616-1703), followed by Abraham de Moivre (1667-1754), and James Stirling (1692-1770). Their work, together with work of Euler, Daniel Bernoulli (1700-1782), and Lagrange (17361813) is reviewed in Dutka 79 .

In 1729, in correspondence with Christian Goldbach, Euler discussed the problem of obtaining continuous interpolations of discrete series. He described a (convergent) infinite product interpolating the factorial function, $m$ !,

$$
\frac{1 \cdot 2^{m}}{1+m} \cdot \frac{2^{1-m} \cdot 3^{m}}{2+m} \cdot \frac{3^{1-m} \cdot 4^{m}}{3+m} \cdot \frac{4^{1-m} \cdot 5^{m}}{4+m} \cdot \text { etc. }
$$

This infinite product, grouped as shown, converges absolutely on the region $\mathbb{C} \backslash$ $\{-1,-2, \ldots\}$, to the gamma function, which we denote $\Gamma(m+1)$, following the later

7 "numerus $n[\ldots]$, et quem per approximationem olim inveni esse $=0,5772156649015325$, cuius valorem nullo adhuc modo ad mensuras transcendentes iam cogitas redigere potui; unde haud inutile erit resolutionem huius formulae propositae pluribus modis tentare."[Translation: Jordan Bell.] 
notation of Legendre. John Wallis (1616-1703) had earlier called this function the "hypergeometric series". Euler substitutes $m=\frac{1}{2}$ and finds

$$
\Gamma\left(\frac{3}{2}\right)=\frac{1}{2} \sqrt{\pi}
$$

by recognizing a relation of this infinite product to Wallis's infinite product for $\frac{\pi}{2}$. In this letter he also introduced the notion of fractional integration; see Sandifer 267.

Also in 1729 Euler [89, E19] wrote a paper presenting these results (On transcendental progressions, that is, those whose general term cannot be given algebraically), but it was not published until 1738. This paper describes the infinite products, suitable for interpolation. It then considers Euler's first integra 8

$$
\int_{0}^{1} x^{e}(1-x)^{n} d x
$$

which in modern terms defines the Beta function $B(e+1, n+1)$, derives a recurrence relation from it, and in Section 9 derives $B(e+1, n+1)=\frac{e ! n !}{(e+n+1) !}$. More generally, in the later notation of Legendre this extends to the Beta function identity

$$
B(r, s):=\int_{0}^{1} x^{r-1}(1-x)^{s-1} d x=\frac{\Gamma(r) \Gamma(s)}{\Gamma(r+s)},
$$

relating it to the gamma function. Euler [89, Section 14] goes on to obtain a continuous interpolation of the factorial function $f(n)=n$ !, given by the integral

$$
f(n)=\int_{0}^{1}(-\log x)^{n} d x
$$

where $n$ is to be interpreted as a continuous variable, $n>-1$. He then computes formulas for $\Gamma\left(1+\frac{n}{2}\right)$ at half-integer values; see [89, Section 20].

In 1765, but not published until 1769, Euler [106, E368] (On a hypergeometric curve expressed by the equation $y=1 * 2 * 3 * \cdots * x)$ studied the "hypergeometric curve" given (using Legendre's notation) by $y=\Gamma(x+1)$, where

$$
\Gamma(x):=\int_{0}^{\infty} t^{x-1} e^{-t} d t .
$$

Euler interpolates the factorial function by an infinite product. In Section 5 he gives the product formula

$$
y=a^{x} \prod_{k=1}^{\infty}\left(\frac{k}{k+x}\left(\frac{a+k}{a+k-1}\right)^{x}\right)
$$

with any fixed $a$ with $1<a<x$, and with $a=\frac{1+x}{2}$ obtains

$$
y=\left(\frac{1+x}{2}\right)^{x} \prod_{k=1}^{\infty}\left(\frac{k}{k+x}\left(\frac{2 k+1+x}{2 k-1+x}\right)^{x}\right) .
$$

In Section 9 he derives a formula, which in modern notation reads

$$
\log y=-\gamma x+\sum_{n=2}^{\infty}(-1)^{n} \frac{\zeta(n)}{n} x^{n},
$$

\footnotetext{
${ }^{8}$ Here the letter $e$ denotes an integer. Only in a later paper, 97, E71], written in 1734, does Euler use the symbol $e$ to mean the constant $2.71828 \cdots$.
} 
where $\gamma$ is Euler's constant. In Section 11 he makes an exponential change of variables from his formula (2.3.2) to obtain the integral formula

$$
y=\int_{0}^{\infty} e^{-v} v^{x} d v
$$

which is (essentially) the standard integral representation for the gamma function. This formula shows that the "hypergeometric curve" is indeed given a: 9

$$
y=\Gamma(x+1) .
$$

In Section 12 he obtains a formula for the digamma function $\psi(x)=\frac{\Gamma^{\prime}}{\Gamma}(x)$, which is

$$
\frac{\Gamma^{\prime}(x+1)}{\Gamma(x+1)}:=\frac{d y}{y d x}=-\gamma+\sum_{k=1}^{\infty} \frac{x}{k(x+k)} .
$$

In formula VII in this section he gives the asymptotic expansion of $\log \Gamma(x+1)$, (Stirling's series), expressed as

$$
\begin{aligned}
\log y= & \frac{1}{2} \log 2 \pi+\left(x+\frac{1}{2}\right) \log x \\
& -x+\frac{A}{2 x}-\frac{1 \cdot 2}{2^{3}} \frac{B}{x^{3}}+\frac{1 \cdot 2 \cdot 3 \cdot 4}{2^{5}} \frac{C}{x^{5}}-\frac{1 \cdot 2 \cdot 3 \cdot 4 \cdot 5 \cdot 6}{2^{7}} \frac{D}{x^{7}}+\text { etc. }
\end{aligned}
$$

with $A=\frac{1}{6}, B=\frac{1}{90}, C=\frac{1}{945}, D=\frac{1}{9450}, \ldots$ Euler also gives here the value of Euler's constant as $\gamma \approx 0,577215664901422$ 5. In Section 14 he finds the explicit value

$$
\Gamma^{\prime}(1):=\left.\frac{d y}{d x}\right|_{x=0}=-\gamma .
$$

In Section 15 he uses $\Gamma\left(\frac{3}{2}\right)=\frac{1}{2} \sqrt{\pi}$ to derive the formula

$$
\frac{\Gamma^{\prime}}{\Gamma}\left(\frac{3}{2}\right)=-\gamma+2-2 \log 2 .
$$

In Section 26 he observes that, for $x=n$ a positive integer and with $a$ treated as a variable,

$$
\Gamma(x+1)=\sum_{k=0}^{\infty}(-1)^{k}\left(\begin{array}{l}
x \\
k
\end{array}\right)(a-k)^{x},
$$

the series terminates at $k=n$, and the resulting function is independent of $a$. This leads him to study in the remainder of the paper the series

$$
s=x^{n}-m(x-1)^{n}+\frac{m(m-1)}{1 \cdot 2}(x-2)^{n}+\frac{m(m-1)(m-2)}{1 \cdot 2 \cdot 3}(x-3)^{n}+\text { etc. }
$$

In this formula he sets $n=m+\lambda$, and he investigates the expansion $s=s(x, \lambda)$ as a function of two variables $(\lambda, x)$ which corresponds to the variables $(x-n, a)$ in (2.3.7). These expansions involve Bernoulli numbers.

\footnotetext{
${ }^{9}$ If one instead used the notation $\Pi(x)$ for this integral used by Gauss (cf. Edwards [81, p. 8]), then the hypergeometric curve is $y=\Pi(x)$. Gauss made an extensive study of hypergeometric functions.
} 
2.4. Zeta values. Already as a young student of Johann Bernoulli (1667-1748) in the 1720s, Euler was shown problems on the summation of infinite series of arithmetical functions, including in particular the sums of inverse $k$ th powers of integers. The problem of finding a closed form expression for this value, $\zeta(2)$, became a celebrated problem called the Basel problem. The Basel problem had been raised by Pietro Mengoli (1628-1686) in his 1650 book on the arithmetical series arising in geometrical quadratures (see [215], [126]). In that book Mengoli [217, Proposition 17] summed in closed form the series of (twice the) inverse triangular numbers

$$
\sum_{n=1}^{\infty} \frac{1}{n(n+1)}=\frac{1}{2}+\frac{1}{6}+\frac{1}{12}+\cdots=1,
$$

which is a telescoping sum. The problem became well known because it was also raised by Johann Bernoulli's older brother Jacob (also called James or Jacques) Bernoulli (1654-1705), earlier holder of the professorship of mathematics at the University of Basel. He wrote a book on infinite series ([29]) in 1689; and several additional works on them. A collection of this work appeared posthumously in 1713 [30]. He could tell that the sum of inverse squares converged to a value between 1 and 2 , using the triangular numbers, via

$$
1<\sum_{n=1}^{\infty} \frac{1}{n^{2}}<2\left(\sum_{n=1}^{\infty} \frac{1}{n(n+1)}\right)=2 \sum_{n=1}^{\infty}\left(\frac{1}{n}-\frac{1}{n+1}\right)=2 .
$$

He was unable to relate this value to known constants, and wrote ( 30 , art. XVII, p. 254]):

And thus by this Proposition, the sums of series can be found when the denominators are either triangular numbers less a particular triangular number or square numbers less a particular square number; by [the results of art.] XV, this happens for pure triangular numbers, as in the series $\frac{1}{1}+\frac{1}{3}+\frac{1}{6}+\frac{1}{10}+\frac{1}{15} \& \mathrm{c}$., while on the other hand, when the numbers are pure squares, as in the series $\frac{1}{1}+\frac{1}{4}+\frac{1}{9}+\frac{1}{16}+\frac{1}{25} \& c$., it is more difficult than one would have expected, which is noteworthy. If someone should succeed in finding what till now withstood our efforts and communicate it to us, we would be much obliged to them 10

Here he says that if $a \geq 1$ is an integer and the denominators range over $n(n+1)-a(a+1)$ or alternatively $n^{2}-a^{2}$, with $n \geq a+1$, then he can sum the series, but he cannot sum the second series when $a=0$.

Euler solved the Basel problem by 1735 with his famous result that

$$
\zeta(2)=\frac{\pi^{2}}{6} .
$$

\footnotetext{
10 "Atque ita per hanc Propositionem, inveniri possunt summae serierum, cum denominatores sunt vel numeri Trigonales minuti alio Trigonali, vel Quadrati minuti alio Quadrato; ut \& per XV. quando sunt puri Trigonales, ut in serie $\frac{1}{1}+\frac{1}{3}+\frac{1}{6}+\frac{1}{10}+\frac{1}{15} \&$ c. at, quod notatu dignum, quando sunt puri Quadrati, ut in serie $\frac{1}{1}+\frac{1}{4}+\frac{1}{9}+\frac{1}{16}+\frac{1}{25}$ \&c difficilior est, quam quis expectaverit, summae pervestigatio, quam tamen finitam esse, ex altera, qua manifesto minor est, colligimus: Si quis inveniat nobisque communicet, quod industriam nostram elusit hactenus, magnas de nobis gratias feret." [Translation: Jordan Bell.]
} 
Euler's first contributions on the Basel problem involved getting numerical approximations of $\zeta(2)$. In [90, E20], written in 1731, integrating his representation for the harmonic numbers led to a representation (in modern terms)

$$
\int_{0}^{1} \frac{d x}{x} \int_{0}^{x} \frac{1-t^{n}}{1-t} d t=\sum_{j=1}^{n} \frac{1}{j^{2}}
$$

By a series acceleration method he obtained the formula

$$
\zeta(2)=(\log 2)^{2}+\sum_{n=1}^{\infty} \frac{1}{2^{n-1} n^{2}},
$$

in which the final sum on the right is recognizable as the dilogarithm value $2 \operatorname{Li}_{2}\left(\frac{1}{2}\right)$. Euler used this formula to calculate $\zeta(2) \approx 1.644924$; cf. Sandifer [267. He next obtained a much better approximation using Euler summation (the Euler-Maclaurin expansion). He announced his summation method in [91, E25] in 1732, and gave details in [94, E47], a paper probably written in 1734. In this paper he computed Bernoulli numbers and polynomials, and also applied his summation formula to obtain $\zeta(2)$ and $\zeta(3)$ to 20 decimal places.

In December 1735, in [92, E41] published in 1740, Euler gave three proofs that $\zeta(2)=\frac{\pi^{2}}{6}$. The most well known of these treated $\sin x$ as though it were a polynomial with infinitely many roots at $\pm \pi, \pm 2 \pi, \pm 3 \pi, \ldots$. He combines these roots in pairs, and asserts the infinite product formula

$$
\frac{\sin x}{x}=\left(1-\frac{x^{2}}{\pi^{2}}\right)\left(1-\frac{x^{2}}{4 \pi^{2}}\right)\left(1-\frac{x^{2}}{9 \pi^{2}}\right) \cdots .
$$

He then equates certain algebraic combinations of coefficients of the Taylor series expansion at $x=0$ on both sides of the equation. The right side coefficients give the power sums of the roots of the polynomials. Writing these Taylor coefficients as $1-\alpha x^{2}+\beta x^{4}-\gamma x^{6}+\cdots$, he creates algebraic combinations giving the power sums of the roots, in effect obtaining 11

$$
\begin{aligned}
\frac{1}{\pi^{2}} \zeta(2) & =\alpha, \\
\frac{1}{\pi^{4}} \zeta(4) & =\alpha^{2}-2 \beta, \\
\frac{1}{\pi^{6}} \zeta(6) & =\alpha^{3}-3 \alpha \beta+3 \gamma,
\end{aligned}
$$

and so on. Now the coefficients $\alpha, \beta, \gamma$ may be evaluated as rational numbers from the power series terms of the expansion of $\frac{\sin x}{x}$ which Euler computes directly. In this way he determined formulas for $\zeta(2 n)$ for $1 \leq n \leq 6$. He also evaluated by a similar approach alternating sums of odd powers, obtaining in Section 10 the result of Leibniz (1646-1716) that

$$
\sum_{k=0}^{\infty}(-1)^{k} \frac{1}{2 k+1}=\frac{\pi}{4}
$$

\footnotetext{
${ }^{11}$ Euler only writes down the right sides of these equations.
} 
He views this as confirming his method, saying:

And indeed this is the same series discovered some time ago by Leibniz, by which he defined the quadrature of the circle. From this, if our method should appear to some as not reliable enough, a great confirmation comes to light here; thus there should not be any doubt about the rest that will be derived from this method 12

In Section 12 he obtained a parallel result for cubes,

$$
\sum_{k=0}^{\infty}(-1)^{k} \frac{1}{(2 k+1)^{3}}=\frac{\pi^{3}}{32}
$$

These proofs are not strictly rigorous, because the infinite product expansion is not completely justified, but Euler makes some numerical cross-checks, and his formulas work. Euler was sure his answer was right from his previous numerical computations. In this same paper he gives two more derivations of the result, still susceptible to criticism, based on the use of infinite products.

We note that directly equating power series coefficients on both sides of (2.4.1), for the the $2 n$-th coefficient would give the identity

$$
\frac{(-1)^{n}}{(2 n+1) !}=\frac{(-1)^{n}}{\pi^{2 n}} \sum_{1 \leq m_{1}<m_{2}<\cdots<m_{n}} \frac{1}{m_{1}^{2} m_{2}^{2} \cdots m_{n}^{2}} .
$$

The sum on the right hand side is the multiple zeta value $\zeta(2,2, \ldots, 2)$ (using $n$ copies of 2 , see (2.4.5) below), and the case $n=1$ gives $\zeta(2)$. Relations like this may have inspired Euler's later study of multiple zeta values.

Euler communicated his original proof to his correspondents Johann Bernoulli, Daniel Bernoulli (1700-1782), Nicolaus Bernoulli (1687-1759), and others. Daniel Bernoulli criticized it on the grounds that $\sin \pi x$ may have other complex roots; cf. [21, Section 4], 315, p. 264]. In response to this criticism Euler eventually justified his infinite product expansion for $\sin \pi x$, obtaining a version that can be made rigorous in modern terms; cf. Sandifer [267.

In a paper [95, E61] published in 1743, Euler obtained more results using infinite products, indicating how to derive formulas for $\zeta(2 n)$, for all $n \geq 1$, as well as for the sums

$$
\left[L\left(2 n+1, \chi_{-4}\right):=\right] \sum_{k=0}^{\infty}(-1)^{k} \frac{1}{(2 k+1)^{2 n+1}},
$$

for $n \geq 0$. In this paper he studied the function $e^{x}=\sum_{n=0}^{\infty} \frac{x^{n}}{n !}$. He recalls his formula for $\sin s$, as

$$
s-\frac{s^{3}}{1 \cdot 2 \cdot 3}+\frac{s^{5}}{1 \cdot 2 \cdot 3 \cdot 4 \cdot 5}-\cdots=s\left(1-\frac{s^{2}}{\pi^{2}}\right)\left(1-\frac{s^{2}}{4 \pi^{2}}\right)\left(1-\frac{s^{2}}{9 \pi^{2}}\right)\left(1-\frac{s^{2}}{16 \pi^{2}}\right) \cdots .
$$

12 "Atque haec est ipsa series a Leibnitio iam pridem prolata, qua circuli quadraturam definiuit. Ex quo magnum huius methodi, si cui forte ea non satis certa videatur, firmamentum elucet; ita ut de reliquis, quae ex hac methodo deriuabantur, omnino non liceat dubitari" [Translation: Jordan Bell.] 
Then he obtains the formula $\sin z=\frac{e^{i z}-e^{-i z}}{2 i}$, introducing the modern notation for $e$, saying:

For, this expression is equal to $\frac{e^{s \sqrt{-1}}-e^{-s \sqrt{-1}}}{2 \sqrt{-1}}$, where $e$ denotes that number whose logarithm $=1$, and

$$
e^{z}=\left(1+\frac{z}{n}\right)^{n}
$$

with $n$ being an infinitely large number 13

Now he introduces a new principle. He evaluates the following integrals, where $p$ and $q$ are integers with $0<p<q$ :

$$
\int_{0}^{1} \frac{x^{p-1}+x^{q-p-1}}{1+x^{q}} d x=\frac{\pi}{q \sin \frac{p \pi}{q}}
$$

and

$$
\int_{0}^{1} \frac{x^{p-1}-x^{q-p-1}}{1-x^{q}} d x=\frac{\pi \cos \frac{p \pi}{q}}{q \sin \frac{p \pi}{q}} .
$$

Expanding the first integral as an indefinite integral in power series in $x$, evaluating term by term, and setting $x=1$, he obtains the formula

$$
\frac{\pi}{q \sin \frac{p \pi}{q}}=\frac{1}{p}+\frac{1}{q-p}-\frac{1}{q+p}-\frac{1}{2 q-p}+\frac{1}{2 q+p}+\frac{1}{3 q-p}-\frac{1}{3 q+p}+\cdots
$$

and similarly for the second integral. Setting $\frac{p}{q}=s$ and rescaling, he obtains

$$
\frac{\pi}{\sin \pi s}=\frac{1}{s}+\frac{1}{1-s}-\frac{1}{1+s}-\frac{1}{2-s}+\frac{1}{2+s}+\frac{1}{3-s}-\cdots
$$

and

$$
\frac{\pi \cos \pi s}{\sin \pi s}=\frac{1}{s}-\frac{1}{1-s}+\frac{1}{1+s}-\frac{1}{2-s}+\frac{1}{2+s}-\frac{1}{3-s}+\cdots .
$$

By differentiating with respect to $s$, he obtains

$$
\frac{\pi^{2} \cos \pi s}{(\sin \pi s)^{2}}=\frac{1}{s^{2}}-\frac{1}{(1-s)^{2}}-\frac{1}{(1+s)^{2}}+\frac{1}{(2-s)^{2}}+\frac{1}{(2+s)^{2}}-\frac{1}{(3-s)^{2}}+\cdots
$$

and

$$
\frac{\pi^{2}}{(\sin \pi s)^{2}}=\frac{1}{s^{2}}+\frac{1}{(1-s)^{2}}+\frac{1}{(1+s)^{2}}+\frac{1}{(2-s)^{2}}+\frac{1}{(2+s)^{2}}+\cdots .
$$

Now he substitutes $s=\frac{p}{q}$ with $0<p<q$ integers and obtains many identities. Thus

$$
\frac{\pi^{2}}{q^{2}\left(\sin \frac{p \pi}{q}\right)^{2}}=\frac{1}{p^{2}}+\frac{1}{(q-p)^{2}}+\frac{1}{(q+p)^{2}}+\frac{1}{(2 q-p)^{2}}+\frac{1}{(2 q+p)^{2}}+\cdots .
$$

For $q=4$ and $p=1$, he obtains in this way

$$
\frac{\pi^{2}}{8 \sqrt{2}}=1-\frac{1}{3^{2}}-\frac{1}{5^{2}}+\frac{1}{7^{2}}+\frac{1}{9^{2}}-\frac{1}{11^{2}}-\frac{1}{13^{2}}+\cdots
$$

and

$$
\frac{\pi^{2}}{8}=1+\frac{1}{3^{2}}+\frac{1}{5^{2}}+\frac{1}{7^{2}}+\frac{1}{9^{2}}+\frac{1}{11^{2}}+\cdots .
$$

13 "Haec enim expressio aequivalet isti $\frac{e^{s \sqrt{-1}}-e^{-s \sqrt{-1}}}{2 \sqrt{-1}}$ denotante $e$ numerorum, cujus logarithmus est $=1, \&$ cum sit $e^{z}=\left(1+\frac{z}{n}\right)^{n}$ existente $n$ numero infinito". [Translation: Jordan Bell.] 
He observes that it is not difficult to derive on like principles the value $\zeta(2)=\frac{\pi^{2}}{6}$. (This identity follows from the observation that the series above is $\left(1-\frac{1}{2^{2}}\right) \zeta(2)$.) He concludes that one may continue differentiating in $s$ to obtain formulas for various series in $n$th powers, and gives several formulas for the derivatives. In this way, one can obtain a formula for $\zeta(2 n)$ that expresses it as a rational multiple of $\pi^{2 n}$. However this approach does not make evident the closed formula for $\zeta(2 n)$ expressed in terms of Bernoulli numbers (given below as (2.4.2) ).

In a paper [96, E63], published in 1743, Euler gave a new derivation of $\zeta(2)=\frac{\pi^{2}}{6}$, using standard methods in calculus involving trigonometric integrals, which is easily made rigorous by today's standards. He takes $s$ to be arclength on a circle of radius 1 and sets $x=\sin s$ so that $s=\arcsin x$. He observes $x=1$ corresponds to $s=\frac{\pi}{2}$, and here he uses the symbol $\pi$ with its contemporary meaning:

It is clear that I employ here the letter $\pi$ to denote the number of Ludolf van Ceulen $3.14159265 \ldots 14$

Here Euler refers to Ludolf van Ceulen (1540-1610), who was a professor at Leiden University and who computed $\pi$ to 35 decimal places. In his calculations Euler works with differentials and writes $d s=\frac{d x}{\sqrt{1-x x}}$, so that $s=\int \frac{d x}{\sqrt{1-x x}}$. Multiplying these expressions gives

$$
s d s=\frac{d x}{\sqrt{1-x x}} \int \frac{d x}{\sqrt{1-x x}} .
$$

He integrates both sides from $x=0$ to $x=1$. The left side is $\int_{0}^{\frac{\pi}{2}} s d s=\frac{\pi \pi}{8}$. For the right side, he uses the binomial theorem

$$
\frac{1}{\sqrt{1-x x}}\left[=(1-x x)^{-\frac{1}{2}}\right]=1+\frac{1}{2} x^{2}+\frac{1 \cdot 3}{2 \cdot 4} x^{4}+\frac{1 \cdot 3 \cdot 5}{2 \cdot 4 \cdot 6} x^{6}+\text { etc. }
$$

In fact this expansion converges for $|x|<1$. Euler integrates it term by term to get

$$
\int \frac{d x}{\sqrt{1-x x}}=x+\frac{1}{2 \cdot 3} x^{3}+\frac{1 \cdot 3}{2 \cdot 4 \cdot 5} x^{5}+\frac{1 \cdot 3 \cdot 5}{2 \cdot 4 \cdot 6 \cdot 7} x^{7}+\text { etc. }
$$

He then obtains

$$
s d s=\frac{x d x}{\sqrt{1-x x}}+\frac{1}{2 \cdot 3} \frac{x^{3} d x}{\sqrt{1-x x}}+\frac{1 \cdot 3}{2 \cdot 4 \cdot 5} \frac{x^{5} d x}{\sqrt{1-x x}}+\frac{1 \cdot 3 \cdot 5}{2 \cdot 4 \cdot 6 \cdot 7} \frac{x^{7} d x}{\sqrt{1-x x}}+\text { etc. }
$$

Integrating from $x=0$ to $x=1$ would give

$$
\frac{\pi \pi}{8}=\int_{0}^{1} \frac{x d x}{\sqrt{1-x x}}+\frac{1}{2 \cdot 3} \int_{0}^{1} \frac{x^{3} d x}{\sqrt{1-x x}}+\frac{1 \cdot 3}{2 \cdot 4 \cdot 5} \int_{0}^{1} \frac{x^{5} d x}{\sqrt{1-x x}}+\text { etc. }
$$

The individual terms on the right can be integrated by parts

$$
\int \frac{x^{n+2} d x}{\sqrt{1-x x}}=\frac{n+1}{n+2} \int \frac{x^{n} d x}{\sqrt{1-x x}}-\frac{x^{n+1}}{n+2} \sqrt{1-x x} .
$$

14 "Il est clair que j'emplois la lettre $\pi$ pour marquer le nombre de LUDOLF à KEULEN 3,14159265 etc." 
When integrating from $x=0$ to $x=1$, the second term on the right vanishes and Euler gives a table

$$
\begin{aligned}
& \int_{0}^{1} \frac{x d x}{\sqrt{1-x x}}=1-\sqrt{1-x x}=1, \\
& \int_{0}^{1} \frac{x^{3} d x}{\sqrt{1-x x}}=\frac{2}{3} \int_{0}^{1} \frac{x d x}{\sqrt{1-x x}}=\frac{2}{3}, \\
& \int_{0}^{1} \frac{x^{5} d x}{\sqrt{1-x x}}=\frac{4}{5} \int_{0}^{1} \frac{x^{3} d x}{\sqrt{1-x x}}=\frac{2 \cdot 4}{3 \cdot 5}, \\
& \int_{0}^{1} \frac{x^{7} d x}{\sqrt{1-x x}}=\frac{6}{7} \int_{0}^{1} \frac{x^{5} d x}{\sqrt{1-x x}}=\frac{2 \cdot 4 \cdot 6}{3 \cdot 5 \cdot 7}, \\
& \int_{0}^{1} \frac{x^{9} d x}{\sqrt{1-x x}}=\frac{8}{9} \int_{0}^{1} \frac{x^{7} d x}{\sqrt{1-x x}}=\frac{2 \cdot 4 \cdot 6 \cdot 8}{3 \cdot 5 \cdot 7 \cdot 9} .
\end{aligned}
$$

Substituting these values in the integration above yields

$$
\frac{\pi \pi}{8}=1+\frac{1}{3 \cdot 3}+\frac{1}{5 \cdot 5}+\frac{1}{7 \cdot 7}+\frac{1}{9 \cdot 9}+\text { etc. }
$$

This gives the sum of reciprocals of odd squares, and Euler observes that multiplying by $\frac{1}{4}$ gives

$$
\frac{1}{4}+\frac{1}{16}+\frac{1}{36}+\frac{1}{64}+\text { etc. }
$$

from which follows

$$
\frac{\pi \pi}{6}=1+\frac{1}{4}+\frac{1}{9}+\frac{1}{16}+\cdots
$$

This proof is rigorous and unarguable.

He goes on to evaluate sums of larger even powers by the same methods, and gives a table of explicit evaluations for $\zeta(2 n)$ for $1 \leq n \leq 13$, in the form

$$
[\zeta(2 n)=] \frac{2^{2 n-1}}{(2 n+1) !} C_{2 n} \pi^{2 n} .
$$

In this expression, he says part of the formula is known and explainable, and that the remaining difficulty is to explain the nature of the fractions $\left[C_{2 n}\right]$ which take values of a different character:

$$
\frac{1}{2} \frac{1}{6} \quad \frac{1}{6} \quad \frac{3}{10} \quad \frac{5}{6} \quad \frac{691}{210} \quad \frac{35}{2} \text { etc. }
$$

He says that he has two other methods for finding these numbers, whose nature he does not specify. As we know, these involve Bernoulli numbers.

In this same period, around 1737, not published until 1744, Euler [99, E72] obtained the "Euler product" expansion of the zeta function. In [99, Theorem 8] he proved:

The expression formed from the sequence of prime numbers

$$
\frac{2^{n} \cdot 3^{n} \cdot 5^{n} \cdot 7^{n} \cdot 11^{n} \cdot \text { etc. }}{\left(2^{n}-1\right)\left(3^{n}-1\right)\left(5^{n}-1\right)\left(7^{n}-1\right)\left(11^{n}-1\right) \text { etc. }}
$$


has the same value as the sum of the series

$$
1+\frac{1}{2^{n}}+\frac{1}{3^{n}}+\frac{1}{4^{n}}+\frac{1}{5^{n}}+\frac{1}{6^{n}}+\frac{1}{7^{n}}+\text { etc } 15
$$

This infinite product formula for $\zeta(n)$ supplies in principle another way to approximate the values $\zeta(n)$ for $n \geq 2$, using finite products.

Euler made repeated attempts throughout his life to find closed forms for the odd zeta values, especially $\zeta(3)$. In a 1739 paper [101, E130], not published until 1750, he developed partial fraction decompositions and a method equivalent to Abel summation. In particular, letting $\theta(s)=\sum_{n=0}^{\infty} \frac{1}{(2 n+1)^{s}}$ and $\phi(s)=\sum_{n=1}^{\infty} \frac{(-1)^{n-1}}{n^{s}}$, he obtained the formula

$$
\phi(1-2 m)=\frac{(-1)^{m-1} 2 \cdot(2 m-1) !}{\pi^{2 m}} \theta(2 m)
$$

for $m=1,2,3,4$, in which the left side is determined as the Abel sum

$$
\phi(m):=\lim _{x \rightarrow 1^{-}} \sum_{n=1}^{\infty} \frac{(-1)^{n-1} x^{n}}{n^{m}} ;
$$

in fact the function $\phi(s)$ is Abel summable for all $s \in \mathbb{C}$.

In a paper written in 1749 [105, E352], not presented until 1761 and published in 1768, Euler derived the functional equation for the zeta function at integer points, and he conjectured

$$
\frac{\phi(1-s)}{\phi(s)}=\frac{-\Gamma(s)\left(2^{s}-1\right) \cos \frac{\pi s}{2}}{\left(2^{s-1}-1\right) \pi^{s}} .
$$

He apparently hoped to use this functional relation to deduce a closed form for the odd zeta values, including $\zeta(3)$, and says:

As far as the [alternating] sum of reciprocals of powers is concerned,

$$
1-\frac{1}{2^{n}}+\frac{1}{3^{n}}-\frac{1}{4^{n}}+\frac{1}{5^{n}}-\frac{1}{6^{n}}+\& \mathrm{c}
$$

I have already observed the sum $[\phi(n)]$ can be assigned a value only when $n$ is even and that when $n$ is odd, all my efforts have been useless up to now 16

In his 1755 book on differential calculus Euler [103, E212, Part II, Chapter 6, Arts. 141-153] gave closed forms for $\zeta(2 n)$ explicitly expressed in terms of the

15 "Si ex serie numerorum primorum sequens formetur espressio

$$
\frac{2^{n} \cdot 3^{n} \cdot 5^{n} \cdot 7^{n} \cdot 11^{n} \cdot \text { etc. }}{\left(2^{n}-1\right)\left(3^{n}-1\right)\left(5^{n}-1\right)\left(7^{n}-1\right)\left(11^{n}-1\right) \text { etc. }}
$$

erit eius aequalis summae huius seriei

$$
1+\frac{1}{2^{n}}+\frac{1}{3^{n}}+\frac{1}{4^{n}}+\frac{1}{5^{n}}+\frac{1}{6^{n}}+\frac{1}{7^{n}}+\text { etc." }
$$

[Translation: David Pengelley [244], 245].] Here $n$ is an integer.

16 "A l'égard des séries réciproques des puissances

$$
1-\frac{1}{2^{n}}+\frac{1}{3^{n}}-\frac{1}{4^{n}}+\frac{1}{5^{n}}-\frac{1}{6^{n}}+\& \mathrm{c}
$$

j'ai déjà observé, que leurs sommes ne sauroient être assignées que lorsque l'exposant $n$ est un nombre entier par, \& que pour les cas ou $n$ est un nombre entier impair, tous mes soins ont été jusqu'ici inutiles." 
Bernoulli numbers. These are equivalent to the modern form

$$
\zeta(2 n)=(-1)^{n+1} \frac{B_{2 n}(2 \pi)^{2 n}}{2(2 n !)} .
$$

He also used his summation formula to numerically evaluate the sums using divergent series, as follows. To estimate $\zeta(2)$ he considers the finite sums

$$
s=1+\frac{1}{4}+\frac{1}{9}+\cdots+\frac{1}{x^{2}}
$$

where $x$ is fixed, and obtains the summation

$$
s=C-\frac{1}{x}+\frac{1}{2 x^{2}}-\frac{\left|B_{2}\right|}{x^{3}}+\frac{\left|B_{4}\right|}{x^{5}}-\frac{\left|B_{6}\right|}{x^{7}}+\cdots,
$$

where the constant $C$ is to be determined by finding all the other terms at one value of $x$. Choosing $x=\infty$, we have $s=\zeta(2)$ and the summation formula formally gives $s=C=\zeta(2)$. On setting $x=1$, the finite sum gives $s=1$, therefore one has, formally,

$$
C=1+1-\frac{1}{2}+\left|B_{2}\right|-\left|B_{4}\right|+\left|B_{6}\right|-\cdots .
$$

The right side of this expression is a divergent series and Euler remarks:

But this series alone does not give the value of $C$, since it diverges strongly. Above [Section 125] we demonstrated that the sum of the series to infinity is $\frac{\pi \pi}{6}$; and therefore setting $x=\infty$ and $s=\frac{\pi \pi}{6}$, we have $C=\frac{\pi \pi}{6}$, because then all other terms vanish. Thus it follows that

$$
1+1-\frac{1}{2}+\left|B_{2}\right|-\left|B_{4}\right|+\left|B_{6}\right|-\cdots=\frac{\pi \pi}{6} 17
$$

Euler's idea is to use these divergent series to obtain good numerical approximations to $C$ by substituting a finite value of $x$ and to add up the terms of the series "until it begins to diverge", i.e. to truncate it according to some recipe. We do not address the question of what rule Euler used to determine where to truncate. This procedure has received modern justification in the theory of asymptotic series; cf. Hardy [156], Hildebrand [166, Section 5.8], [81, Sec 6.4]. By taking large $x$, one can get arbitrarily accurate estimates (for some functions). If the series is alternating, one may sometimes estimate the error as being no larger than the smallest term. Euler demonstrates in this particular case how to find the value $C=\frac{\pi^{2}}{6}$ numerically using the value $x=10$, with $s=\sum_{i=1}^{10} \frac{1}{n^{2}}$, by truncating the expansion (2.4.3) after power $\frac{1}{x^{17}}$, obtaining $C \approx 1,644934066848226430$. He writes:

And this number is at once the value of the expression $\frac{\pi^{2}}{6}$, as will be apparent by doing the calculation with the known value

\footnotetext{
17 "quae series autem cum sit maxime divergens, valorem constantis $C[:=\zeta(2)]$ non ostendit. Quia autem supra demonstavimus summam huius seriei in infinitum continuatae esse $=\frac{\pi \pi}{6}$; facto $x=\infty$, si ponatur $s=\frac{\pi \pi}{6}$, fiet $C=\frac{\pi \pi}{6}$, ob reliquos terminos omnes evanescentes. Erit ergo

$$
1+1-\frac{1}{2}+\mathfrak{U}-\mathfrak{B}+\mathfrak{C}-\mathfrak{D}+\mathfrak{E}-\& c=\frac{\pi \pi}{6} .^{\prime \prime}
$$
}

[Translation: David Pengelley [244, Section 148], 245].] 
of $\pi$. Whence it is at once understood that even though the series $\left[\left|B_{2}\right|,\left|B_{4}\right|,\left|B_{6}\right|, \ldots\right]$ diverges, still the true sum is produced 18

Euler also applies this method to the odd zeta values, obtaining numerical approximations for all zeta values up to $\zeta(15)$, in particular

$$
\zeta(3) \approx \frac{\pi^{3}}{25.79436}
$$

He also (implicitly) introduces in Section 153 a "Bernoulli function" $b(z)$ which interpolates at integer values the variable $z$ his version of the even Bernoulli numbers, i.e.

Euler remarks:

$$
b(n)=\left|B_{2 n}\right|=(-1)^{n+1} B_{2 n}, n \in \mathbb{Z}_{\geq 1} .
$$

The series of Bernoulli numbers $\left[\left|B_{2}\right|,\left|B_{4}\right|,\left|B_{6}\right|, \ldots\right]$ however irregular, seems to be interpolated from this source; that is, terms constituted in the middle of two of them can be defined: for if the middle term lying between the first $A$ and the second $B$, corresponding to the index $1 \frac{1}{2}$ were $=p$, then it would certainly be

and hence

$$
1+\frac{1}{2^{3}}+\frac{1}{3^{3}}+\& \mathrm{tc}=\frac{2^{2} p}{1 \cdot 2 \cdot 3} \pi^{3}
$$

$$
p=\frac{3}{2 \pi^{3}}\left(1+\frac{1}{2^{3}}+\frac{1}{3^{3}}+\& c\right)=0,0581522719
$$

That is, Euler shows the interpolating value $p:=b\left(\frac{3}{2}\right)$ is related to $\zeta(3)$, by the relation

$$
p=\frac{3}{2} \frac{\zeta(3)}{\pi^{3}}
$$

and he finds the numerical value $p \approx 0.05815227$. He similarly obtains a value for $b\left(\frac{5}{2}\right)$ in terms of $\zeta(5)$.

In a 1771 paper, published in 1776, Euler [109, E477] (Meditations about a singular type of series) introduced multiple zeta values and with them obtained another formula for $\zeta(3)$. He had considered this topic much earlier. In December 1742 Goldbach wrote Euler a letter about multiple zeta values, and they exchanged several further letters on their properties. The 1771 paper recalls this correspondence with Goldbach, and considers the sums, for $m, n \geq 1$ involving the $n$-harmonic numbers

$$
S_{m, n}:=\sum_{k=1}^{\infty} \frac{H_{k, n}}{k^{m}}=\sum_{k \geq 1}\left(\sum_{1 \leq l \leq k} \frac{1}{k^{m} l^{n}}\right)=\sum_{l \geq 1} \sum_{k \geq l}\left(\frac{1}{k^{m} l^{n}}\right) .
$$

Euler lets $\int \frac{1}{z^{m}}$ denote $\zeta(m)$ and lets $\int \frac{1}{z^{m}}\left(\frac{1}{z}\right)^{n}$ denote the sum $S_{m, n}$. He considers all values $1 \leq m, n \leq 8$. This includes divergent series, since all sums $S_{1, n}$ are

\footnotetext{
18 "Hicque numerus simul est valor expressionis $\frac{\pi \pi}{6}$, quemadmodem ex valore ipsius $\pi$ cogito calculum institutenti patebit. Unde simul intelligitur, etiamsi series $\mathfrak{A}, \mathfrak{B}, \mathfrak{C} \&$ c. divergat, tamen hoc modo veram prodire summan." [Translation: Jordan Bell.]

19 "Ex hoc fonte series numerorum Bernoulliarum $\begin{array}{ccc}1 & 2 & 3 \\ \mathfrak{U} & \mathfrak{B} & \mathfrak{C}\end{array}[\cdots]$ quantumvis irregularis videatur interpolari, seu termini in medio binorum quorumcunque constituti assignari poterunt; se enim terminus medium interiacens inter primum $\mathfrak{A}$ and secundum $\mathfrak{B}$, sue indici $1 \frac{1}{2}$, respondens fuerit $=p$; erit utique $1+\frac{1}{2^{3}}+\frac{1}{3^{3}}+\&$ tc. $=\frac{2^{2} p}{1 \cdot 2 \cdot 3} \pi^{3}$ ideoque $p=\frac{3}{2 \pi^{3}}\left(1+\frac{1}{2^{3}}+\frac{1}{3^{3}}+\& c\right)=0,05815227 . "$ [Translation: Jordan Bell.]
} 
divergent. The modern definition of multiple zeta values, valid for positive integers $\left(a_{1}, \ldots, a_{k}\right)$ with $a_{1} \geq 2$, is

$$
\zeta\left(a_{1}, a_{2}, \ldots, a_{k}\right)=\sum_{n_{1}>n_{2}>\cdots>n_{k}>0} \frac{1}{n_{1}^{a_{1}} n_{2}^{a_{2}} \cdots n_{k}^{a_{k}}} .
$$

In this notation (2.4.4) expresses the identities (valid for $m \geq 2, n \geq 1$ )

$$
S_{m, n}=\zeta(n+m)+\zeta(m, n) .
$$

Euler presents three methods for obtaining identities, and establishes a number of additional identities for each range $m+n=j$ for $2 \leq j \leq 11$. For $j=3$ he obtains

$$
S_{2,1}=2 \zeta(3) \text {. }
$$

This is equivalent to

$$
\zeta(3)=\zeta(2,1)=\frac{1}{2}\left(\sum_{k=1}^{\infty} \frac{H_{k}}{k^{2}}\right) .
$$

Being thorough, Euler also treats cases $m=1$ where the series diverge, and observes that for $j=2$ he cannot derive useful identities.

In a 1772 paper [108, E432] (Analytical exercises) published in 1773, Euler obtained new formulas for values $\zeta(2 n+1)$ via divergent series related to the functional equation for the zeta function. As an example, in Section 6 he writes, treating $n$ as a positive integer and $\omega$ as an infinitely small quantity, the formula

$$
1+\frac{1}{3^{n+\omega}}+\frac{1}{5^{n+\omega}}+\cdots=\frac{-1}{2 \cos \left(\frac{n+\omega}{2} \pi\right)} \frac{\pi^{n+\omega}}{1 \cdot 2 \cdots(n-1+\omega)}\left(1-2^{n-1+\omega}+3^{n-1+\omega}-\text { etc. }\right)
$$

Here the right side contains a divergent series. In Section 7 , he in effect takes a derivative in $\omega$ and obtains, for example in the case $n=3$, the formula

$$
1+\frac{1}{3^{3}}+\frac{1}{5^{3}}+\frac{1}{7^{3}}+\text { etc. }=-\frac{\pi^{2}}{2}\left(2^{2} \log 2-3^{2} \log 3+4^{2} \log 4-5^{2} \log 5+\text { etc. }\right)
$$

The right side is another divergent series, which he names

$$
Z:=2^{2} \log 2-3^{2} \log 3+4^{2} \log 4-5^{2} \log 5+6^{2} \log 6-7^{2} \log 7+\text { etc. }
$$

He applies various series acceleration techniques on this divergent series; compare the discussion of divergent series in Section 2.5 below. In Section 20 he transforms $Z$ into a rapidly convergent series,

$$
Z=\frac{1}{4}-\frac{\alpha \pi^{2}}{3 \cdot 4 \cdot 2^{2}}-\frac{\beta \pi^{4}}{5 \cdot 6 \cdot 2^{4}}-\frac{\gamma \pi^{6}}{7 \cdot 8 \cdot 2^{6}}-\frac{\delta \pi^{8}}{9 \cdot 10 \cdot 2^{8}}-\frac{\epsilon \pi^{10}}{11 \cdot 12 \cdot 2^{10}}-\text { etc. }
$$

In his notation $\zeta(2)=\alpha \pi^{2}, \zeta(4)=\beta \pi^{4}$, etc., so he thus obtains the valid, rapidly convergent formula

$$
\frac{7}{8} \zeta(3)=\frac{\pi^{2}}{2}\left(\frac{1}{4}-\sum_{n=1}^{\infty} \frac{\zeta(2 n)}{(2 n+1)(2 n+2) 2^{2 n}}\right) .
$$

After further changes of variable, he deduces in Section 21 the formula

$$
1+\frac{1}{3^{3}}+\frac{1}{5^{3}}+\frac{1}{7^{3}}+\text { etc. }=\frac{\pi^{2}}{4} \log 2+2 \int_{0}^{\frac{\pi}{2}} \Phi \log (\sin \Phi) d \Phi .
$$

This may be rewritten

$$
\frac{7}{8} \zeta(3)=\frac{\pi^{2}}{4} \log 2+2 \int_{0}^{\frac{\pi}{2}} x \log (\sin x) d x .
$$


Euler notes also the "near miss" that a variant of the last integral is exactly evaluable:

$$
\int_{0}^{\frac{\pi}{2}} \log (\sin x) d x=-\frac{\pi}{2} \log 2 .
$$

Euler continued to consider zeta values. In the 1775 paper [111, E597] ( $A$ new and most easy method for summing series of reciprocals of powers) published posthumously in 1785 , he gave a new derivation of his formulas for the even zeta values $\zeta(2 n)$. In the 1779 paper [113, E736] published in 1811, he studied the dilogarithm function $\operatorname{Li}_{2}(x):=\sum_{n=1}^{\infty} \frac{x^{n}}{n^{2}}$. In Section 4 he establishes the functional equation

$$
\operatorname{Li}_{2}(x)+\operatorname{Li}_{2}(1-x)=\frac{\pi^{2}}{6}-(\log x)(\log (1-x)) .
$$

The special case $x=0$ of this functional equation gives again $\zeta(2)=\frac{\pi^{2}}{6}$. Euler deduces several other functional equations for the dilogarithm, and by specialization, a number of new series identities.

2.5. Summing divergent series: the Euler-Gompertz constant. In the course of his investigations Euler encountered many divergent series. His work on divergent series was a byproduct of his aim to obtain accurate numerical estimates for the sum of various convergent series, and he learned and developed summability methods which could be applied to both convergent and divergent series. By cross-checking predictions of various summability methods, he developed useful methods to sum divergent series. Of particular interest here, Euler's summation of a particular divergent series, the Wallis hypergeometric series, produced an interesting new constant, the Euler-Gompertz constant.

Euler's near contemporary James Stirling (1692-1770) developed methods of acceleration of convergence of series by differencing, starting in 1719, influenced by Newton's work ([234, pp. 92-101]) on interpolation; see Tweddle [307. Euler obtained Stirling's 1730 book 291, translated in 308 (Differential methods and summation of series), which discussed infinite series, summation, interpolation and quadrature, in which Proposition 14 gives three examples of a method of accelerating convergence of series of hypergeometric type. On 8 June 1736 Euler wrote to Stirling, saying ([308, p. 229], [306, p. 141]):

I searched all over for your excellent book on the method of differences, a review of which I had seen in the Acta Lipenses, until I have achieved my desire. Now that I have read through it diligently, I am truly astonished at the great abundance of excellent methods in such a small volume, by which you show to sum slowly convergent series with ease and how to interpolate progressions which are very difficult to deal with. But especially pleasing to me was Proposition 14 of Part I, in which you present a method for summing so easily series whose law of progression is not even established, using only the relation of the last terms; certainly this method applies very widely and has the greatest use. But the demonstration of this proposition, which you seemed to have concealed from study, caused me immense difficulty, until at last with the greatest pleasure I obtained it from the things which had gone before... 
A key point is that Proposition 14 applies when the members $a_{n}$ of the series satisfy linear recurrence relations whose coefficients are rational functions of the parameter $n$. This point was not made explicit by Stirling. It is known that there exist counterexamples to Proposition 14 otherwise (cf. Tweddle [308, pp. 229-233]). The convergence acceleration method of Stirling is really a suite of methods of great flexibility, as is explained by Gosper [136, 20 Euler incorporated these ideas in his subsequent work 21

In a 1760 paper [104, E247] (On divergent series) Euler formulated his views on the use and philosophy of assigning a finite value to some divergent series. This paper includes work Euler had done as early as 1746. In it Euler presents four different methods for summing the particular divergent series

$$
1-1+2-6+24-120+\cdots[=0 !-1 !+2 !-3 !+4 !-5 !+\cdots],
$$

which he calls "Wallis' hypergeometric series", and shows they each give (approximately) the same answer, which he denotes $A$. Barbeau and Leah [26] give a translation of the initial, philosophical part of this paper, followed by a summary of the rest of its contents, and Barbeau 27] gives a detailed exposition of Euler's treatment of this example.

The series (2.5.1) is termed "hypergeometric" because each term in the series is multiplied by a ratio which varies from term to term, rather than by a constant ratio as in a geometric series. Series of this type appeared in the Scholium to Proposition 190 in Wallis's 1656 book Arithmetica Infinitorum 314,22

Euler's four methods were:

(1) [104, Section 13-16] The iteration of a series acceleration method involving a differencing operation applied to this series. Euler iterates four times, truncates the resulting divergent series, and obtains an approximation to its "sum" $A$ of $A \approx 38015 / 65536=0.58006 \cdots$.

(2) [104, Section 17-18] The interpolation of solutions to the difference equation $P_{n+1}=n P_{n}+1$, with $P_{1}=1$. Euler obtains the closed formula

$$
P_{n}=1+(n-1)+(n-1)(n-2)+(n-1)(n-2)(n-3)+\cdots,
$$

which is a finite sum for each $n \geq 1$, and observes that substituting $n=0$ formally gives the Wallis series. He considers interpolation methods that rescale $P_{n}$ by an invertible function $f(x)$ to obtain values $f\left(P_{n}\right)$, and then attempts to interpolate the value $f\left(P_{0}\right)$. For the choice $f(x)=\frac{1}{x}$ he obtains $A \approx 0.6$ and for $f(x)=\log x$ he obtains $A \approx 0.59966$.

\footnotetext{
${ }^{20}$ Gosper 136 p.122] says: "We will be taking up almost exactly where James Stirling left off, and had he been granted use of a symbolic mathematics system such as MIT MACSYMA, Stirling would probably have done most of this work before 1750."

${ }^{21}$ We do not attempt to untangle the exact influence of Stirling's work on Euler. In the rest of his 1736 letter Euler explains his own Euler summation method for summing series. In a reply made 16 April 1738, Stirling tells Euler that Colin Maclaurin will be publishing a book on Fluxions that has a similar summation formula that he found some time ago. Euler replied on 27 July 1738 that "Mr. Maclaurin probably came upon his summation formula before me, and consequently deserves to be named as its first discoverer. For I found that theorem about four years ago, at which time I also described its proof and application in greater detail to our Academy." Euler also states that he independently found some summability methods in Stirling's book, before he knew of its existence ([306, Chapter 6]).

${ }^{22}$ This reference was noted by Carl Boehm, an editor of Euler's Collected Works; cf. 26, p. 157].
} 
(3) [104, Section 19-20] The construction and solution of an associated differential equation. Euler views the Wallis series as the special case $x=1$ of the power series

$$
s(x)=1-1 ! x+2 ! x^{2}-3 ! x^{3}+4 ! x^{4}-5 ! x^{5}+\cdots .
$$

Although this series diverges for all nonzero $x \in \mathbb{C}$, he observes that $s(x)$ formally satisfies a linear differential equation. This differential equation has a convergent solution given by an integral with variable endpoint $x$, and by specializing it to $x=1$, he obtains an exact answer $A$. We discuss this method in detail below.

(4) 104, Section 21-25] The determination of a continued fraction expansion for the series $s(x)$ given by the integral in (3). This is

$$
s(x)=\frac{1}{1+\frac{x}{1+\frac{x}{1+\frac{2 x}{1+\frac{2 x}{1+\frac{3 x}{1+\frac{3 x}{1+\cdots}}}}}}}
$$

This continued fraction is convergent at $x=1$. Using it, he computes a good numerical approximation, given in (2.5.8) below, to the answer $A$.

In the third of four summation methods, Euler considers the series to be the "value" at $x=1$ of the asymptotic series

$$
s(x) \sim \sum_{n=0}^{\infty}(-1)^{n} n ! x^{n+1} .
$$

He notes this series satisfies the inhomogeneous linear differential equation

$$
s^{\prime}(x)+\frac{1}{x^{2}} s(x)=\frac{1}{x} .
$$

He writes down an explicit solution to the differential equation (2.5.4), namely

$$
s(x):=e^{\frac{1}{x}} \int_{0}^{x} \frac{1}{t} e^{-\frac{1}{t}} d t .
$$

The expansion of this integral near $x=0$ has the asymptotic expansion (2.5.3). Euler transforms the integral (2.5.5) into other forms. Using the change of variable $v=e^{1-\frac{1}{t}}$, he obtains

$$
s(x)=e^{\left(\frac{1}{x}-1\right)} \int_{0}^{e^{1-\frac{1}{x}}} \frac{d v}{1-\log v} .
$$

He then obtains as his proposed summation for the divergent series the value

$$
\delta:=s(1)=e \int_{0}^{1} \frac{1}{t} e^{-\frac{1}{t}} d t=\int_{0}^{1} \frac{d v}{1-\log v} .
$$

He numerically estimates this integral using the trapezoidal rule and obtains $\delta \approx$ 0.59637255. Using his fourth method, in Section 25 Euler obtained a more precise 
numerical value for this constant, finding

$$
\delta \approx 0.596347362 \underline{1} 237 .
$$

The constant $\delta$ defined by (2.5.7) is of some interest and we call it the EulerGompertz constant, following Finch [116, Section 6.2]. This constant was earlier named the Gompertz constant by Le Lionnais 203. 23. Benjamin Gompertz (17791865) was a mathematician and actuary who in 1825 proposed a functional model for approximating mortality tables; cf. Gompertz [132, [133]. The Gompertz function and Gompertz distribution are named in his honor. I have not located the Euler-Gompertz constant in his writings; however, Finch [116, Section 6.2.4] points out a connection of the Euler-Gompertz constant with a Gompertz-like functional model.

The Euler-Gompertz constant has some properties analogous to Euler's constant. The integral representations for the Euler-Gompertz constant given by (2.5.5) and (2.5.6) evaluated at $x=1$ resemble the integral representations (2.2.9) and (2.2.10) for Euler's constant. An elegant parallel was noted by Aptekarev [13],

$$
\gamma=-\int_{0}^{\infty} \log x e^{-x} d x, \quad \delta=\int_{0}^{\infty} \log (x+1) e^{-x} d x
$$

There are also integral formulae in which both constants appear; see (3.5.11) and (3.6.8).

In 1949 G. H. Hardy [156, p. 26] gave a third integral representation for the function $s(x)$, obtained by using the change of variable $t=\frac{x}{1+x w}$, namely

$$
s(x)=x \int_{0}^{\infty} \frac{e^{-w}}{1+x w} d w .
$$

Evaluation at $x=1$ yields the integral representation

$$
\delta=\int_{0}^{\infty} \frac{e^{-w}}{1+w} d w
$$

From this representation Hardy obtained a relation of $\delta$ to Euler's constant given by

$$
\delta=-e\left(\gamma-1+\frac{1}{2 \cdot 2 !}-\frac{1}{3 \cdot 3 !}+\frac{1}{4 \cdot 4 !}+\cdots\right) .
$$

One can also deduce from (2.5.10) that

$$
\delta=-e \operatorname{Ei}(-1)
$$

where $E i(x)$ is the exponential integral

$$
\operatorname{Ei}(x):=\int_{-\infty}^{x} \frac{e^{t}}{t} d t
$$

which is defined as a function of a complex variable $x$ on the complex plane cut along the nonnegative real axis 24

\footnotetext{
${ }^{23}$ Le Lionnais 203 gives no explanation or reference for his choice of name "Gompertz".

${ }^{24}$ This is sufficient to define $E i(-1)$. For applications the function $E i(x)$ is usually extended to positive real values by using the Cauchy principal value at the point $x=0$ where the integrand has a singularity. The real-valued definition using the Cauchy principal value is related to the complex-analytic extension by

$$
E i(x):=\lim _{\epsilon \rightarrow 0^{+}} \frac{1}{2}(E i(x+\epsilon i)+E i(x-\epsilon i)) .
$$
}


Here we note that Euler's integral (2.5.5) is transformed by the change of variable $t=\frac{1}{u}$ to another simple form, which on replacing $x$ by $1 / x$ becomes

$$
s\left(\frac{1}{x}\right)=e^{x} \int_{x}^{\infty} \frac{e^{-u}}{u} d u=e^{x} E_{1}(x) .
$$

Here $E_{1}(z)$ is the principal exponential integral, defined for $|\arg z|<\pi$ by a contour integral

$$
E_{1}(z):=\int_{z}^{\infty} \frac{e^{-u}}{u} d u
$$

that eventually goes to $+\infty$ along the positive real axis; see [1, Chapter 5]. Choosing $x=1$ yields the identity

$$
E_{1}(1)=\int_{1}^{\infty} \frac{e^{-t}}{t} d t=\frac{\delta}{e}
$$

Further formulas involving the Euler-Gompertz constant are given in Section 3.5, Section 3.6. Section 3.15, and Section 3.16.

Regarding method (4) above, Euler had previously done much work on continued fractions in the late 1730s in [97, E71] and in particular in his sequel paper [100, E123], in which he expands some analytic functions and integrals in continued fractions. This work of Euler is discussed in the book of Khrushchev [175, which includes an English translation of [100]. In 1879 E. Laguerre [193, found an analytic continued fraction expansion for the principal exponential integral, which coincides with $e^{-x} s(1 / x)$ by (2.5.14), obtaining

$$
\int_{x}^{\infty} \frac{e^{-t}}{t} d t=\frac{e^{-x}}{x+1-\frac{1}{x+3-\frac{1}{\frac{x+5}{4}-\frac{\frac{1}{4}}{\frac{x+7}{9}-\frac{\frac{1}{9}}{\frac{x+9}{16}-\cdots} .}}}}
$$

Laguerre noted that this expansion converges for positive real $x$. His expansion simplifies th 25

$$
\int_{x}^{\infty} \frac{e^{-t}}{t} d t=\frac{e^{-x}}{x+1-\frac{1^{2}}{x+3-\frac{2^{2}}{x+5-\frac{3^{2}}{x+7-\frac{4^{2}}{x+9-\cdots}}}}}
$$

\footnotetext{
${ }^{25}$ This form of fraction with minus signs between convergents is often called a $J$-continued fraction; see Wall [313, p. 103].
} 
which is the form in which it appears in Wall [313, (92.7)]. On taking $x=1$, it yields another continued fraction for the Euler-Gompertz constant,

$$
\delta=e \int_{1}^{\infty} \frac{e^{-t}}{t} d t=\frac{1}{2-\frac{1^{2}}{4-\frac{2^{2}}{6-\frac{3^{2}}{8-\frac{4^{2}}{10-\cdots}}}}}
$$

In his great 1894 work T. J. Stieltjes (289], 290]) developed a general theory for analytic continued fractions representing functions $F(x)$ given by $\int_{0}^{\infty} \frac{d \Phi(u)}{x+u}$, which takes the form

$$
\int_{0}^{\infty} \frac{d \Phi(u)}{x+u}=\frac{1}{a_{1} x+\frac{1}{a_{2}+\frac{1}{a_{3} x+\frac{1}{a_{4}+\frac{1}{a_{5} x+\frac{1}{a_{6}+\cdots}}}}}}
$$

for positive real $a_{n}$. He formulated the (Stieltjes) moment problem for measures on the nonnegative real axis, and related his continued fractions to the moment problem. In the introduction of his paper he specifically gives Laguerre's continued fraction (2.5.18) as an example, and observes that it may be converted to a continued fraction in his form (2.5.20) with $a_{2 n-1}=1$ and $a_{2 n}=\frac{1}{n}$. On taking $x=1$ his converted fraction is closely related to Euler's continued fraction (2.5.2). Stieltjes notes that his theory extends the provable convergence region for Laguerre's continued fraction (2.5.18) to all complex $x$, excluding the negative real axis. In [289, Section 57] he remarks that associated measure in the moment problem for this continued fraction must be unbounded, and in [289, Section 62] he obtains a generalization of (his form of) Laguerre's continued fraction to add extra parameters; cf. Wall [313, (92.17)].

It is not known whether the Euler-Gompertz constant is rational or irrational. However it has recently been established that at least one of $\gamma$ and $\delta$ is transcendental, as presented in Section 3.16 .

2.6. Euler-Mascheroni constant; Euler's approach to research. Euler's constant is often called the Euler-Mascheroni constant, after later work of Lorenzo Mascheroni [213], 214]. In 1790, in a book proposing to answer some problems raised by Euler, Mascheroni 213 introduced the notation $A$ for this constant. In 1792 Mascheroni [214, p. 11] also reconsidered integrals in Euler's paper [112, E629], using both the notations $A$ and $a$ for Euler's constant. In his book, using an expansion of the logarithmic integral, he gave a numerical expression for it to 32 decimal places ([213, p. 23]), which stated

$$
A \approx 0.5772156649015328606 \underline{1} 811209008239 .
$$

This computation attached his name to the problem; Euler had previously computed it accurately to 15 places. At that time constants were often named after a 
person who had done the labor of computing them to the most digits. However in 1809 Johann von Soldner 275, while preparing the first book on the logarithmic integral function $\operatorname{li}(x)=\int \frac{d x}{\log x}$, found it necessary to recompute Euler's constant, which he called $H$ (perhaps from the harmonic series). He computed it to 22 decimal places by a similar method, and obtained ([275, p. 13])

$$
H \approx 0.5772156649015328606065 .
$$

Von Soldner's answer disagreed with Mascheroni's in the twentieth decimal place. To settle the conflict, von Soldner asked the aid of C. F. Gauss, and Gauss engaged the 19-year old calculating prodigy F. G. B. Nicolai (1793-1846) to recheck the computation. Nicolai used the Euler-Maclaurin summation formula to compute $\gamma$ to 40 places, finding agreement with von Soldner's calculations to 22 places (see [158, p. 89]). Thus the conflict was settled in von Soldner's favor, and Mascheroni's value is accurate to only 19 places. In the current era, which credits the mathematical contributions, it seems most appropriate to name the constant after Euler alone.

The now-standard notation $\gamma$ for Euler's constant arose subsequent to the work of both Euler and Mascheroni. Euler himself used various notations for his constant, including $C$ and $O$ and $n$; Mascheroni used $A$ and $a$; von Soldner used $H$ and $C$. The notation $\gamma$ appears in an 1837 paper on the logarithmic integral of Bretschneider [45. p. 260]. It also appears in a calculus textbook of Augustus de Morgan [226. p. 578], published about the same time. This choice of symbol $\gamma$ is perhaps based on the association of Euler's constant with the gamma function.

To conclude our discussion of Euler's work, it seems useful to contemplate the approach to research used by Euler in his long successful career. C. Truesdell 303 , Essay 10], 304, pp. 91-92], makes the following observations about the methods used by Euler, his teacher Johann Bernoulli, and Johann's teacher and brother Jacob Bernoulli:

(1) Always attack a special problem. If possible solve the special problem in a way that leads to a general method.

(2) Read and digest every earlier attempt at a theory of the phenomenon in question.

(3) Let a key problem solved be a father to a key problem posed. The new problem finds its place on the structure provided by the solution of the old; its solution in turn will provide further structure.

(4) If two special problems solved seem cognate, try to unite them in a general scheme. To do so, set aside the differences, and try to build a structure on the common features.

(5) Never rest content with an imperfect or incomplete argument. If you cannot complete and perfect it yourself, lay bare its flaws for others to see.

(6) Never abandon a problem you have solved. There are always better ways. Keep searching for them, for they lead to a fuller understanding. While broadening, deepen and simplify.

Truesdell speaks here about Euler's work in the area of foundations of mechanics, but his observations apply equally well to Euler's work in number theory and analysis discussed above. 


\section{Mathematical DEVELOPMENTS}

We now consider a collection of mathematical topics in which Euler's constant appears.

3.1. Euler's constant and the gamma function. Euler's constant appears in several places in connection with the gamma function. Already Euler noted a basic occurrence of Euler's constant with the gamma function,

$$
\Gamma^{\prime}(1)=-\gamma
$$

Thus $\gamma$ appears in the Taylor series expansion of $\Gamma(z)$ around the point $z=1$. Furthermore Euler's constant also appears (in the form $e^{\gamma}$ ) in the Hadamard product expansion for the entire function $\frac{1}{\Gamma(z)}$, which states:

$$
\frac{1}{\Gamma(z)}=z e^{\gamma z} \prod_{n=1}^{\infty}\left(1+\frac{z}{n}\right) e^{-\frac{z}{n}} .
$$

Euler's constant, however, seems more tightly associated to the logarithm of the gamma function, which was studied by Euler [106, E368] and many subsequent workers. The function $\log \Gamma(z)$ is multivalued under analytic continuation, and many results are more elegantly expressed in terms of the digamma function, defined by

$$
\psi(z):=\frac{\Gamma^{\prime}(z)}{\Gamma(z)}=\frac{d}{d z} \log \Gamma(z) .
$$

The theory of the digamma function is simpler in some respects because it is a meromorphic function. Nielsen [239, Chapter 1] presents results and some early history, and Ferraro [115] describes the work of Gauss on these functions.

We recall a few basic facts. The digamma function satisfies the difference equation

$$
\psi(z+1)=\psi(z)+\frac{1}{z}
$$

which is inherited from the functional equation $z \Gamma(z)=\Gamma(z+1)$. It has an integral representation, valid for $\operatorname{Re}(z)>0$,

$$
\psi(z)=\int_{0}^{\infty}\left(\frac{e^{-t}}{t}-\frac{e^{-z t}}{1-e^{-t}}\right) d t,
$$

found in 1813 by Gauss [124, Art. $30 \mathrm{ff}$.] as part of his investigation of the hypergeometric function ${ }_{2} F_{1}(\alpha, \beta ; \gamma ; x)$ (see [115]). The difference equation gives

$$
\psi(x)=\psi(x+n)-\sum_{j=0}^{n-1} \frac{1}{x+j}
$$

from which follows

$$
\psi(x)=\lim _{n \rightarrow \infty}\left(\log n-\sum_{j=0}^{n-1} \frac{1}{x+j}\right) .
$$

This limit is valid for all $x \in \mathbb{C} \backslash \mathbb{Z}_{\leq 0}$, using the fact that $\psi(x)-\log x$ goes uniformly to 0 in the right half-plane as $\operatorname{Re}(x) \rightarrow \infty$ ([239, pp. 85, 93]). This formula gives $\psi(1)=-\gamma$, a fact which also follows directly from (3.1.1).

Euler's constant appears in the values of the digamma function $\psi(x)$ at all positive integers and half-integers. In 1765 Euler [106, E368] found the values $\Gamma\left(\frac{1}{2}\right), \Gamma(1)$ 
and $\Gamma^{\prime}\left(\frac{1}{2}\right), \Gamma^{\prime}(1)$. These values determine $\psi(1 / 2), \psi(1)$, and then, by using the difference equation (3.1.3), the values of $\psi(x)$ for all half-integers.

Theorem 3.1.1 (Euler 1765). The digamma function $\psi(x):=\frac{\Gamma^{\prime}(x)}{\Gamma(x)}$ satisfies, for integers $n \geq 1$,

$$
\psi(n)=-\gamma+H_{n-1},
$$

using the convention that $H_{0}=0$. It also satisfies, for half-integers $n+\frac{1}{2}$, and $n \geq 0$,

$$
\psi\left(n+\frac{1}{2}\right)=-\gamma-2 \log 2+2 H_{2 n-1}-H_{n-1},
$$

using the additional convention $H_{-1}=0$.

Proof. The first formula follows from $\psi(1)=-\gamma$ using (3.1.3). The second formula will follow from the identity

$$
\psi\left(\frac{1}{2}\right)=-\gamma-2 \log 2,
$$

because the difference equation (3.1.3) then gives

$$
\psi\left(n+\frac{1}{2}\right)=\psi\left(\frac{1}{2}\right)+2\left(1+\frac{1}{3}+\cdots+\frac{1}{2 n-1}\right) .
$$

To obtain (3.1.4), we start from the duplication formula for the gamma function

$$
\Gamma(2 z)=\frac{1}{\sqrt{\pi}} 2^{2 z-1} \Gamma(z) \Gamma\left(z+\frac{1}{2}\right) .
$$

We logarithmically differentiate this formula at $z=\frac{1}{2}$, and solve for $\psi\left(\frac{1}{2}\right)$.

The next two results present expansions of the digamma function that connect it with integer zeta values. The first one concerns its Taylor series expansion around the point $z=1$.

Theorem 3.1.2 (Euler 1765). The digamma function has Taylor series expansion around $z=1$ given by

$$
\psi(z+1)=-\gamma+\sum_{k=1}^{\infty}(-1)^{k+1} \zeta(k+1) z^{k} .
$$

This expansion converges absolutely for $|z|<1$.

Remark. The pattern of Taylor coefficients in (3.1.6) has the $k=0$ term formally assigning to " $\zeta(1)$ " the value $\gamma$.

Proof. Euler [106, E368, Section 9] obtained a Taylor series expansion around $z=1$ for $\log \Gamma(z)$, given as (2.3.3) (with $x=z-1$ ). Differentiating it term by term with respect to $x$ yields (3.1.6).

The second relation to zeta values concerns the asymptotic expansion of the digamma function around $z=+\infty$, valid in the right half-plane. In this formula the nonpositive integer values of the zeta function appear.

Theorem 3.1.3. The digamma function $\psi(z)$ has the asymptotic expansion

$$
\psi(z+1) \sim \log z+\sum_{k=1}^{\infty}(-1)^{k} \zeta(1-k)\left(\frac{1}{z}\right)^{k},
$$


which is valid on the sector $-\pi+\epsilon \leq \arg z \leq \pi-\epsilon$ for any given $\epsilon>0$. This asymptotic expansion does not converge for any value of $z$.

The statement that (3.1.7) is an asymptotic expansion means: For each finite $n \geq 1$, the estimate

$$
\psi(z+1)=\log z+\sum_{k=1}^{n}(-1)^{k} \zeta(1-k)\left(\frac{1}{z}\right)^{k}+O\left(z^{-n-1}\right)
$$

holds on the sector $-\pi+\epsilon \leq \arg z \leq \pi-\epsilon$, with $\epsilon>0$ where the implied constant in the $O$-symbol depends on both $n$ and $\epsilon$.

Theorem 3.1.3 does not seem to have been previously stated in this form, with coefficients containing $\zeta(1-k)$. However it is quite an old result, stated in the alternate form

$$
\psi(z+1) \sim \log z-\frac{B_{1}}{z}-\sum_{k=2}^{\infty} \frac{B_{2 k}}{2 k} \frac{1}{z^{2 k}} .
$$

The conversion from (3.1.8) to (3.1.7) is simply a matter of substituting the formulae for the zeta values at negative integers: $\zeta(0)=-\frac{1}{2}=B_{1}$ and $\zeta(1-k)=(-1)^{k+1} \frac{B_{2 k}}{2 k}$, noting $B_{2 k+1}=0$ for $k \geq 1$; see [87, Section 1.18], [1, (6.3.18)]. As a formal expansion (3.1.8) follows either from Stirling's expansion of sums of logarithms obtained in 1730 ([291, Proposition 28], cf. Tweddle [306, Chapter 1]), or from Euler's expansion of $\log \Gamma(z)$ given in (2.3.5), by differentiation term by term. Its rigorous interpretation as an asymptotic expansion having a remainder term estimate valid on the large region given above is due to Stieltjes. In his 1886 French doctoral thesis Stieltjes 287 treated a general notion of an asymptotic expansion with remainder term, including the case of an asymptotic expansion for $\log \Gamma(a)$ (287, Sections 14-19]). In 1889 Stieltjes [288 presented a detailed derivation of an asymptotic series for $\log \Gamma(a)$, whose methods can be used to derive the result (3.1.8) above.

Proof. The 1889 formula of Stieltjes 288 for $\log \Gamma(a)$ embodies Stirling's expansion with an error estimate. Stieltjes first introduced the function $J(a)$ defined by

$$
\log \Gamma(a)=\left(a-\frac{1}{2}\right) \log a-a+\frac{1}{2} \log (2 \pi)+J(a) .
$$

His key observation is that $J(a)$ has the integral representation

$$
J(a)=\int_{0}^{\infty} \frac{P(x)}{x+a} d x
$$

in which $P(x)$ is the periodic function

$$
P(x)=\frac{1}{2}-x+\lfloor x\rfloor=-B_{1}(x-\lfloor x\rfloor),
$$

where $B_{1}(x)$ denotes the first Bernoulli polynomial. He observes that the integral formula (3.1.9) has the merit that it converges on the complex $a$-plane aside from the negative real axis, unlike two earlier integral formulas for $J(a)$ of Binet,

$$
J(a)=\int_{0}^{\infty}\left(\frac{1}{1-e^{-x}}-\frac{1}{x}-\frac{1}{2}\right) \frac{e^{-a x}}{x} d x
$$

and

$$
J(a)=\frac{1}{\pi} \int_{0}^{\infty} \frac{a}{a^{2}+x^{2}} \log \left(\frac{1}{1-e^{-2 \pi x}}\right) d x
$$


which are valid on the half-plane $\operatorname{Re}(a)>0$. He now repeatedly integrates (3.1.9) by parts to obtain a formula 26 for $\log \Gamma(a)$, valid on the entire complex plane aside from the negative real axis,

$$
\begin{aligned}
\log \Gamma(a)= & \left(a-\frac{1}{2}\right) \log a-a+\frac{1}{2} \log (2 \pi) \\
& +\frac{\left|B_{2}\right|}{1 \cdot 2 a}-\frac{\left|B_{4}\right|}{3 \cdot 4 a^{3}}+\cdots+\frac{(-1)^{k-1}\left|B_{2 k}\right|}{(2 k-1) 2 k a^{2 k-1}}+J_{k}(a),
\end{aligned}
$$

in which $J_{k}(a)=\int_{0}^{\infty} \frac{P_{k}(x)}{(x+a)^{2 k+1}} d x$ where $P_{k}(x)$ is periodic and given by its Fourier series

$$
P_{k}(x)=(-1)^{k}(2 k) ! \sum_{n=1}^{\infty} \frac{1}{2^{2 k}(\pi n)^{2 k+1}} \sin (2 \pi n x)
$$

and is related to the $(2 k+1)$-st Bernoulli polynomial. He obtains an error estimate for $J_{k}(a)$ ([288, (34)]), valid for $-\pi+\epsilon<\theta<\pi-\epsilon$, that

$$
\left|J_{k}\left(\operatorname{Re}^{i \theta}\right)\right|<\frac{\left|B_{2 k+2}\right|}{(2 k+2)(2 k+1)}\left(\sec \frac{1}{2} \theta\right)^{2 k+2} R^{-2 k-1} .
$$

We obtain a formula for $\psi(a)$ by differentiating (3.1.10), and using $B_{2 k}=$ $(-1)^{k-1}\left|B_{2 k}\right|$ gives

$$
\psi(a)=\log a-\frac{1}{2 a}-\frac{B_{2}}{2 a^{2}}-\frac{B_{4}}{4 a^{4}}-\cdots-\frac{B_{2 k}}{2 k a^{2 k}}+J_{k}^{\prime}(a) .
$$

This formula gives the required asymptotic expansion for $\psi(a)$, as soon as one obtains a suitable error estimate for $J_{k}^{\prime}(a)$, which may be done on the same lines as for $J_{k}(a)$. The asymptotic expansion (3.1.8) follows using $\psi(a+1)=\psi(a)+\frac{1}{a}$, which has the effect of reversing the sign of the coefficient of $\frac{1}{a}$.

The formula (3.1.7) follows by direct substitution of the zeta values $\zeta(0)=-\frac{1}{2}=$ $B_{1}$ and $\zeta(1-k)=(-1)^{k+1} \frac{B_{k}}{k}$ for $k \geq 2$, noting $B_{2 k+1}=0$ for $k \geq 1$; see [87, Section 1.18], [1, (6.3.18)].

Finally, the known growth rate for even Bernoulli numbers,

$$
\left|B_{2 n}\right| \sim \frac{2}{(2 \pi)^{2 n}}(2 n) ! \sim 4 \sqrt{\pi n}(\pi e)^{-2 n} n^{2 n},
$$

implies that the asymptotic series (3.1.7) diverges for all finite values of $z$.

A consequence of the asymptotic expansion (3.1.7) for $\psi(x)$ is an asymptotic expansion for the harmonic numbers, given by

$$
H_{n} \sim \log n+\gamma+\sum_{k=1}^{\infty}(-1)^{k} \zeta(1-k) \frac{1}{n^{k}} .
$$

This is obtained on taking $z=n$ in (3.1.6) and substituting $\psi(n+1)=H_{n}-\gamma$. Substituting the zeta values allows this expansion to be expressed in the more well known form

$$
\begin{aligned}
H_{n} & \sim \log n+\gamma-\frac{B_{1}}{n}-\sum_{k=2}^{\infty} \frac{B_{2 k}}{2 k} \frac{1}{n^{2 k}} \\
& \sim \log n+\gamma+\frac{1}{2 n}-\frac{1}{12 n^{2}}+\frac{1}{120 n^{4}}-\frac{1}{252 n^{6}}+\cdots
\end{aligned}
$$

\footnotetext{
${ }^{26}$ In Stieltjes's formula $B_{j}$ denotes the Bernoulli number $\left|B_{2 j}\right|$ in 2.2.6). We have altered his notation to match (2.2.6).
} 
Note that although $H_{n}$ has jumps of size $\frac{1}{n}$ at each integer value, nevertheless the asymptotic expansion is valid to all orders $\left(\frac{1}{n}\right)^{k}$ for $k \geq 1$, although the higher order terms are of magnitude much smaller than the jumps.

Theorem 3.1.2 and Theorem 3.1.3 (2) have the feature that these two expansions exhibit between them the full set of integer values $\zeta(k)$ of the Riemann zeta function, with Euler's constant appearing as a substitute for " $\zeta(1)$ ". This is a remarkable fact.

3.2. Euler's constant and the zeta function. The function $\zeta(s)$ was studied at length by Euler, but is named after Riemann [259, commemorating his epochmaking 1859 paper. Riemann showed that $\zeta(s)$ extends to a meromorphic function in the plane with its only singularity being a simple pole at $s=1$. Euler's constant appears in the Laurent series expansion of $\zeta(s)$ around the point $s=1$, as follows.

Theorem 3.2.1 (Stieltjes 1885). The Laurent expansion $\zeta(s)$ around $s=1$ has the form

$$
\zeta(s)=\frac{1}{s-1}+\gamma_{0}+\sum_{n=1}^{\infty} \frac{(-1)^{n}}{n !} \gamma_{n}(s-1)^{n},
$$

in which $\gamma_{0}=\gamma$ is Euler's constant, and the coefficients $\gamma_{n}$ for $n \geq 0$ are defined by

$$
\gamma_{n}:=\lim _{m \rightarrow \infty}\left(\sum_{k=1}^{m} \frac{(\log k)^{n}}{k}-\frac{(\log m)^{n+1}}{n+1}\right) .
$$

Proof. We follow a proof of Bohman and Fröberg [36]. It starts from the identity $(s-1) \zeta(s)=\sum_{k=1}^{\infty}(s-1) k^{-s}$, which is valid for $\operatorname{Re}(s)>1$. For real $s>1$, one has the telescoping sum

Using it, one obtains

$$
\sum_{k=1}^{\infty}\left(k^{1-s}-(k+1)^{1-s}\right)=1
$$

$$
(s-1) \zeta(s)=1+\sum_{k=1}^{\infty}\left\{(k+1)^{1-s}-k^{1-s}+(s-1) k^{-s}\right\} .
$$

Noting that at $s=1$ the telescoping sum above is 0 , one has

$$
\begin{gathered}
(s-1) \zeta(s)=1+\sum_{k=1}^{\infty}\{\exp (-(s-1) \log (k+1))-\exp (-(s-1) \log k) \\
\left.+(s-1) \frac{1}{k} \exp (-(s-1) \log k)\right\} \\
=1+\sum_{k=1}^{\infty}\left\{\sum_{n=0}^{\infty} \frac{(-1)^{n}(s-1)^{n}}{n !}\left[(\log (k+1))^{n}-(\log k)^{n}\right]\right. \\
\left.+\frac{s-1}{k} \sum_{n=0}^{\infty} \frac{(-1)^{n}(s-1)^{n}(\log k)^{n}}{n !}\right\} .
\end{gathered}
$$

Dividing by $(s-1)$ now yields

$$
\zeta(s)=\frac{1}{s-1}+\sum_{n=0}^{\infty} \frac{(-1)^{n}}{n !} \gamma_{n}(s-1)^{n},
$$


with $\gamma_{0}=\gamma$, and with

$$
\gamma_{n}=\sum_{k=1}^{\infty}\left\{\frac{(\log k)^{n}}{k}-\frac{(\log (k+1))^{n+1}-(\log k)^{n+1}}{n+1}\right\} .
$$

This last formula is equivalent to (3.2.2), since the first $m$ terms in the sum add up to the $m$ th term in (3.2.2).

Theorem 3.2.1 gives the coefficients $\gamma_{n}$ of the zeta function in a form resembling the original definition of Euler's constant. These constants arose in a correspondence that Stieltjes carried on with his mentor Hermite (cf. [23]) from 1882 to 1894. In Letter 71 of that correspondence, written in June 1885, Stieltjes stated that he proposed to calculate the first few terms in the Laurent expansion 27 of $\zeta(s)$ around $s=1$,

$$
\zeta(z+1)=\frac{1}{z}+A_{0}+A_{1} z+\cdots,
$$

noting that $A_{0}=0.577215665 \cdots$ is known and that he finds $A_{1}=-0.072815 \underline{5} 20 \cdots$. In Letters 73 and 74, Hermite formulated (3.2.2), which he called "very interesting", and inquired about rigorously establishing it 28 In Letter 75 Stieltjes [23, pp. 151155] gave a complete derivation of (3.2.2), writing

$$
\zeta(s+1)=\frac{1}{s}+C_{0}-C_{1} s+\frac{C_{2}}{1 \cdot 2} s^{2}-\frac{C_{3}}{1 \cdot 2 \cdot 3} x^{3}+\cdots .
$$

The Laurent series coefficients $A_{n}$ are now named the Stieltjes constants by some authors who follow Letter 71 ([36], 39]), while other authors follow Letter 75 and

\begin{tabular}{|c|c|}
\hline$n$ & $\gamma_{n}$ \\
\hline 0 & +0.577215664901532 \\
\hline 1 & - 0.072815845483676 \\
\hline 2 & -0.009690363128723 \\
\hline 3 & +0.002053834420303 \\
\hline 4 & +0.002325370065467 \\
\hline 5 & + 0.000793323817301 \\
\hline 6 & -0.000238769345430 \\
\hline 7 & -0.000527289567057 \\
\hline 8 & - 0.000352123353803 \\
\hline 9 & - 0.000034394774418 \\
\hline 10 & + 0.000205332814909 \\
\hline
\end{tabular}
call the scaled values $\gamma_{n}=C_{n}$ the Stieltjes constants ([173], [59]).

TABLE 1. Values for Stieltjes constants $\gamma_{n}$.

Table 1 presents values of $\gamma_{n}$ for small $n$ as computed by Keiper [173. A good deal is known about the size of these constants. There are infinitely many $\gamma_{n}$ of each sign (Briggs [46]). Because $\zeta(s)-\frac{1}{s-1}$ is an entire function, the Laurent coefficients $A_{n}$ must rapidly approach 0 in absolute value as $n \rightarrow \infty$. However despite the sizes of the initial values in Table 1, the scaled values $\gamma_{n}$ are known

\footnotetext{
${ }^{27}$ In Letter 71 Stieltjes uses the notation $C_{0}, C_{1}, \ldots$, but in Letter 75 he uses the same notation with a different meaning. We substitute $A_{0}, A_{1}, \ldots$ for the Letter 71 coefficients following [36].

${ }^{28}$ The result (3.2.2) is misstated in Erdëlyi et al. [87, (1.12.17)], making the right side equal $\frac{(-1)^{n}}{n !} \gamma_{n}$, a mistake tracing back to a paper of G. H. Hardy 154 .
} 
to be unbounded (and to sometimes be exponentially large) as a function of $n$; cf. Cohen [62, Section 10.3.5]. Keiper estimated that $\gamma_{150} \approx 8.02 \times 10^{35}$. More detailed asymptotic bounds on the $\gamma_{n}$ are given in Knessl and Coffey [177]; see also Coffey 60. At present nothing is known about the irrationality or transcendence of any of the Stieltjes constants.

Various generalizations of the Riemann zeta function play a role in theoretical physics, especially in quantum field theory, in connection with a method of "zeta function regularization" for assigning finite values in certain perturbation calculations. This method was suggested by S. Hawking [159] in 1977, and it has now been widely extended to other questions in physics; see the book of Elizalde 82 . and also [83], 84]. Here one forms a generalized "zeta function" from spectral data and evaluates it an an integer point, often $s=0$. If the function is meromorphic at this point, then the constant term in the Laurent expansion at this value is taken as the "regularized" value. This procedure can be viewed as a quite special kind of "dimensional regularization", in which perturbation calculations are made viewing the dimension of space-time as a complex variable $s$, and at the end of the calculation one specializes the complex variable to the integer value of the dimension, often $s=4$; see Leibbrandt 202]. Under this convention Euler's constant $\gamma$ functions as a "regularized" value " $\zeta(1)$ " of the zeta function at $s=1$, because by Theorem 3.2 .1 it is the constant term in the Laurent expansion of the Riemann zeta function at $s=1$. This interpretation as " $\zeta(1)$ " also matches the constant term in the Taylor expansion of the digamma function $\psi(z)$ around $z=1$ in Theorem 3.1.2

Euler's constant occurs in many deeper ways in connection with the behavior of the Riemann zeta function. The most tantalizing open problem about the Riemann zeta function is the Riemann hypothesis, which asserts that the nonreal complex zeros of the Riemann zeta function all fall on the line $\operatorname{Re}(s)=\frac{1}{2}$. Sections 3.7 and 3.8 present formulations of the Riemann hypothesis in terms of the relation of limiting asymptotic behaviors of arithmetic functions to $e^{\gamma}$. Section 3.9 presents results relating the extreme value distribution of the Riemann zeta function on the line $\operatorname{Re}(s)=1$ to the constant $e^{\gamma}$.

In 1885 Stieltjes announced a proof of the Riemann hypothesis ([286, English translation in [290, p. 561]). In Letter 71 to Hermite discussed above, Stieltjes enclosed his announcement of a proof of the Riemann hypothesis, requesting that it be communicated to Comptes Rendus, if Hermite approved, and Hermite did so. In Letter 79, written later in 1885, Stieltjes sketched his approach, saying that his proof was very complicated and that he hoped to simplify it. Stieltjes considered the Mertens function

$$
M(n)=\sum_{j=1}^{n} \mu(j),
$$

in which $\mu(j)$ is the Möbius function (defined in Section 3.3) and asserted that he could prove that $|M(n)| / \sqrt{n}$ is a bounded function, a result which would certainly imply the Riemann hypothesis. Stieltjes had further correspondence in 1887 with Mittag-Leffler, also making the assertion that he could prove $\frac{M(n)}{\sqrt{n}}$ is bounded between two limits; see [23, Tome II, Appendix], 258, Section 2]. He additionally made numerical calculation: 29 of the Mertens function $M(n)$ for ranges $1 \leq n \leq$

\footnotetext{
${ }^{29}$ Such calculations were found among his posthumous possessions, according to te Riele [258] p. $69]$.
} 
1200, $2000 \leq n \leq 2100$, and $6000 \leq n \leq 7000$, verifying it was small over these ranges. However he never produced a written proof of the Riemann hypothesis related to this announcement. Modern work relating the Riemann zeta zeros and random matrix theory now indicates that $|M(n)| / \sqrt{n}$ should be an unbounded function, although this is unproved; see te Riele [258, Section 4] and Edwards [81, Section 12.1].

3.3. Euler's constant and prime numbers. Euler's constant appears in various statistics associated to prime numbers. In 1874 Franz Mertens 219] determined the growth behavior of the sum of reciprocals of prime numbers, as follows.

Theorem 3.3.1 (Mertens's Sum Theorem 1874). For $x \geq 2$,

$$
\sum_{p \leq x} \frac{1}{p}=\log \log x+B+R(x),
$$

with $B$ being a constant

$$
B=\gamma+\sum_{m=2}^{\infty} \mu(m) \frac{\log \zeta(m)}{m} \approx 0.2614972129 \ldots
$$

and with remainder term bounded by

$$
|R(x)| \leq \frac{4}{\log (P+1)}+\frac{2}{P \log P},
$$

where $P$ denotes the largest prime smaller than $x$.

Here $\mu(n)$ is the Möbius function, which takes the values $\mu(1)=1$ and $\mu(n)=$ $(-1)^{k}$ if $n$ is a product of $k$ distinct prime factors, and $\mu(n)=0$ otherwise. This result asserts the existence of the constant

$$
B:=\lim _{x \rightarrow \infty}\left(\sum_{p \leq x} \frac{1}{p}-\log \log x\right),
$$

which is then given explicitly by (3.3.1). Mertens's proof deduced that $B=\gamma-H$ with

$$
H:=-\sum_{p}\left\{\log \left(1-\frac{1}{p}\right)+\frac{1}{p}\right\}=0.3157184519 \cdots .
$$

For modern derivations which determine the constant $B$, see Hardy and Wright [157. Theorem 428] or Tenenbaum and Mendes France [294, Section 1.9].

Mertens [219, Section 3] deduced from this result the following product theorem, involving Euler's constant alone.

Theorem 3.3.2 (Mertens's Product Theorem 1874). One has

$$
\lim _{x \rightarrow \infty}(\log x) \prod_{p \leq x}\left(1-\frac{1}{p}\right)=e^{-\gamma} .
$$

More precisely, for $x \geq 2$,

$$
\prod_{p \leq x}\left(1-\frac{1}{p}\right)=\frac{e^{-\gamma+S(x)}}{\log x}
$$


in which

$$
|S(x)| \leq \frac{4}{\log (P+1)}+\frac{2}{P \log P}+\frac{1}{2 P},
$$

where $P$ denotes the largest prime smaller than $x$. Thus $S(x) \rightarrow 0$ as $x \rightarrow \infty$.

In this formula the constant $e^{-\gamma}$ encodes a property of the entire ensemble of primes in the following sense: if one could vary a single prime in the product above, holding the remainder fixed, then the limit on the right side of (3.3.3) would change.

In Mertens's product theorem the finite product

$$
D(x):=\prod_{p \leq x}\left(1-\frac{1}{p}\right)
$$

is the inverse of the partial Euler product for the Riemann zeta function, taken over all primes below $x$, evaluated at the point $s=1$. The number $D(x)$ gives the limiting density of integers having no prime factor smaller than $x$, taken on the interval $[1, T]$ and letting $T \rightarrow \infty$ while holding $x$ fixed. Now the set of integers below $T=x^{2}$ having no prime smaller than $x$ consists of exactly the primes between $x$ and $x^{2}$. Letting $\pi(x)$ count the number of primes below $x$, the prime number theorem gives $\pi(x) \sim \frac{x}{\log x}$, and this yields the asymptotic formula

$$
\pi\left(x^{2}\right)-\pi(x) \sim \frac{x^{2}}{\log x^{2}}-\frac{x}{\log x} \sim \frac{1}{2} \frac{x^{2}}{\log x} .
$$

On the other hand, if the density $D(x)$ in Merten's theorem were asymptotically correct already at $T=x^{2}$, then it would predict the number of such primes to be about $e^{-\gamma} \frac{x^{2}}{\log x}$. The fact that $e^{-\gamma}=0.561145 \cdots$ does not equal $\frac{1}{2}$, but is slightly larger, reflects the failure of the inclusion-exclusion formula to give an asymptotic formula in this range. This is a basic difficulty studied by sieve methods.

A subtle consequence of this failure is that there must be occasional unexpectedly large fluctuations in the number of primes in short intervals away from their expected number. This phenomenon was discovered by H. Maier [210] in 1985. It is interesting to note that Maier's argument used a "double counting" argument in a tabular array of integers that resembles the array summations of Euler and Bernoulli exhibited in Section 2.2. His argument also used properties of the Buchstab function discussed in Section 3.6. See Granville [139] for a detailed discussion of this large fluctuation phenomenon.

3.4. Euler's constant and arithmetic functions. Euler's constant also appears in the behavior of three basic arithmetic functions, the divisor function $d(n)$, Euler's totient function $\phi(n)$, and the sum of divisors function $\sigma(n)$. For the divisor function $d(n)$ it arises in its average behavior, while for $\phi(n)$ and $\sigma(n)$ it concerns extremal asymptotic behavior and there appears in the form $e^{\gamma}$. These extremal behaviors can be deduced starting from Mertens's results.

A basic arithmetic function is the divisor function

$$
d(n):=\#\{d: d \mid n, 1 \leq d \leq n\},
$$

which counts the number of divisors of $n$. In 1849 Dirichlet [75] introduced a method to estimate the average size of the divisor function and other arithmetic functions. In the statement of Dirichlet's result, $\{x\}:=x-[x]$ denotes the fractional part $x(\bmod 1)$. 
Theorem 3.4.1 (Dirichlet 1849). (1) The partial sums of the divisor function satisfy

$$
\sum_{k=1}^{n} d(k)=n \log n+(2 \gamma-1) n+O(\sqrt{n})
$$

for $1 \leq n<\infty$, where $\gamma$ is Euler's constant.

(2) The averages of the fractional parts of $\frac{n}{k}$ satisfy

$$
\sum_{k=1}^{n}\left\{\frac{n}{k}\right\}=(1-\gamma) n+O(\sqrt{n})
$$

for $1 \leq n<\infty$.

Proof. Dirichlet's proof starts from the identity

$$
\sum_{k=1}^{n} d(k)=\sum_{k=1}^{n}\left\lfloor\frac{n}{k}\right\rfloor,
$$

which relates the divisor function to integer parts of a scaled harmonic series. The right side of this identity is approximated using the harmonic sum estimate

$$
\sum_{k=1}^{n} \frac{n}{k}=n \log n+\gamma n+O(1)
$$

in which Euler's constant appears. The difference term is the sum of fractional parts. In Section 2 Dirichlet formulates the "hyperbola method" to count the sum of the lattice points on the right side of (3.4.3), and in Section 3 he applies the method to get part (1). In Section 4 he compares the answer (3.4.1) with (3.4.3) and obtains the formula (3.4.2) giving part (2). A modern treatment of the hyperbola method appears in Tenenbaum [293, I.3.2].

The problem of obtaining bounds for the size of the remainder term in (3.4.1),

$$
\Delta(n):=\sum_{k=1}^{n} d(k)-(n \log n+(2 \gamma-1) n),
$$

is now called the Dirichlet divisor problem. This problem seeks the best possible bound for the exponent $\theta$ of the form $|\Delta(n)|=O\left(n^{\theta+\epsilon}\right)$, valid for any given $\epsilon>0$ for $1 \leq n<\infty$, with $O$-constant depending on $\epsilon$. This problem appears to be very difficult. Dirichlet's argument shows that the size of the remainder term $\Delta(n)$ is dominated by the remainder term in the fractional part sum (3.4.2). In 1916 Hardy [155] established the lower bound $\theta \geq \frac{1}{4}$. The exponent $\theta=1 / 4$ is conjectured to be the correct answer and would correspond to an expected square-root cancellation. The best $\Omega$-result on fluctuations of $\Delta(n)$, which does not change the lower bound exponent, is that of Soundararajan [283] in 2003. Concerning upper bounds, a long sequence of improvements in exponent bounds has arrived at the current record $\theta \leq \frac{131}{416} \approx 0.31490$, obtained by Huxley $([168$, , 169]) in 2005. Tsang [302] gives a recent survey on recent work on $\Delta(n)$ and related problems.

Dirichlet noted that (3.4.2) implies that the fractional parts $\left\{\left\{\frac{n}{k}\right\} ; 1 \leq k \leq n\right\}$ are far from being uniformly distributed $(\bmod 1)$. In Section 4 of $[75]$ he proved that the set of fractional parts $k$ having $0 \leq\left\{\frac{n}{k}\right\} \leq \frac{1}{2}$ has a limiting frequency $(2-\log 4) n=(0.61370 \cdots) n$. and those with $\frac{1}{2}<\left\{\frac{n}{k}\right\}<1$ have the complementary density $(\log 4-1) n=(0.38629 \cdots) n$, as $n \rightarrow \infty$. 
In 1898 de La Vallée Poussin [189] generalized the fractional part sum estimate (3.4.2) above to summation over $k$ restricted to an arithmetic progression. He showed for each integer $a \geq 1$ and integer $0<b \leq a$,

$$
\sum_{k=0}^{(n-b) / a}\left\{\frac{n}{a k+b}\right\}=\frac{1}{a}(1-\gamma) n+O(\sqrt{n}),
$$

for $1 \leq n<\infty$, with $O$-constant depending on $a$. He comments that it is very remarkable that these fractional parts approach the same average value $1-\gamma$, no matter which arithmetic progression we restrict them to. Very recently Pillichshammer [246] presented further work in this direction.

We next consider Euler's totient function

$$
\phi(n):=\#\{j: 1 \leq j \leq n, \text { with } \operatorname{gcd}(j, n)=1\},
$$

which has $\phi(n)=\left|(\mathbb{Z} / n \mathbb{Z})^{*}\right|$. Euler's constant appears in the asymptotics of the minimal growth rate (minimal order) of $\phi(n)$. In 1903 Edmund Landau [195] showed the following result, which he also presented later in his textbook [196, pp. 216-219].

Theorem 3.4.2 (Landau 1903). Euler's totient function $\phi(n)$ has minimal order $\frac{n}{\log \log n}$. Explicitly,

$$
\liminf _{n \rightarrow \infty} \frac{\phi(n) \log \log n}{n}=e^{-\gamma}
$$

Since $\phi(n)=n \prod_{p \mid n}\left(1-\frac{1}{p}\right)$, its extremal behavior is attained by the primorial numbers, which are the numbers that are products of the first $k$ primes, i.e. $N_{k}:=$ $p_{1} p_{2} \cdots p_{k}$, for example $N_{4}=2 \cdot 3 \cdot 5 \cdot 7=210$. The result then follows using Mertens's product theorem.

The sum of divisors function is given by

$$
\sigma(n):=\sum_{d \mid n} d
$$

so that e.g. $\sigma(6)=12$. Euler's constant appears in the asymptotics of the maximal growth rate (maximal order) of $\sigma(n)$. In 1913 Gronwall [145] obtained the following result.

Theorem 3.4.3 (Gronwall 1913). The sum of divisors function $\sigma(n)$ has maximal order $n \log \log n$. Explicitly,

$$
\limsup _{n \rightarrow \infty} \frac{\sigma(n)}{n \log \log n}=e^{\gamma} .
$$

For $\sigma(n)$ the extremal numbers form a very thin set, with a more complicated description, which was analyzed by Ramanujan 253. in 1915 (see also Ramanujan [255]) and Alaoglu and Erdös [3] in 1944. They have the property that their divisibility by each prime $p^{e_{p}(n)}$ has exponent $e_{p}(n) \rightarrow \infty$ as $n \rightarrow \infty$; see Section 3.7 and formula (3.7.3).

The Euler totient function $\phi(n)$ and the sum of divisors function $\sigma(n)$ are related by the inequalities

$$
\frac{6}{\pi^{2}} n^{2}<\phi(n) \sigma(n) \leq n^{2}
$$


which are easily derived from the identity 30

$$
\phi(n) \sigma(n)=n^{2} \prod_{\substack{p^{e} \| n \\ e \geq 1}}\left(1-\frac{1}{p^{e+1}}\right) .
$$

We note that the ratio of extremal behaviors of the "smallest" $\phi(n)$ (resp. the "largest" $\sigma(n))$ given in Theorems 3.4.2 and 3.4.3, have a product asymptotically growing like $n^{2}$. The extremal numbers $N_{k}=p_{1} p_{2} \cdots p_{k}$ in Landau's theorem are easily shown to satisfy $\phi\left(N_{k}\right) \sigma\left(N_{k}\right) \sim \frac{6}{\pi^{2}} N_{k}^{2}$ as $N_{k} \rightarrow \infty$. The extremal numbers $\tilde{n}$ in Gronwall's theorem can be shown to satisfy the other bound

$$
\phi(\tilde{n}) \sigma(\tilde{n}) \sim \tilde{n}^{2}
$$

as $\tilde{n} \rightarrow \infty$. The latter result implies that the Gronwall extremal numbers satisfy

$$
\lim _{\tilde{n} \rightarrow \infty} \frac{\phi(\tilde{n}) \log \log \tilde{n}}{\tilde{n}}=e^{-\gamma},
$$

showing they are asymptotically extremal in Landau's theorem as well.

In Sections 3.7 and 3.8 we discuss more recent results showing that the Riemann hypothesis is encoded as an assertion as to how the extremal limits are approached in Landau's theorem and Gronwall's theorem.

3.5. Euler's constant and sieve methods: the Dickman function. Euler's constant appears in connection with statistics on the sizes of prime factors of general integers. Let $\Psi(x, y)$ count the number of integers $n \leq x$ having largest prime factor no larger than $y$. These are the numbers remaining if one sieves out all integers divisible by some prime exceeding $y$. The Dirichlet series associated to the set of integers having no prime factor $>y$ is the partial zeta function

$$
\zeta(s ; y):=\prod_{p \leq y}\left(1-\frac{1}{p^{s}}\right)^{-1}
$$

and we have

$$
\zeta(1 ; y)=\frac{1}{D(y)}
$$

where $D(y)$ is given in (3.3.4). It was shown by Dickman [73] in 1930 that, for each $u>0$, a positive fraction of all integers $n \leq x$ have all prime factors less than $x^{1 / u}$. More precisely, one has the asymptotic formula

$$
\Psi\left(x, x^{\frac{1}{u}}\right) \sim \rho(u) x,
$$

for a certain function $\rho(u)$, now called the Dickman function. The notation $\rho(u)$ was introduced by de Bruijn [50, [51]. This function is determined for $u \geq 0$ by the following properties.

(1) Initial condition. For $0 \leq u \leq 1$, it satisfies

$$
\rho(u)=1 \text {. }
$$

(2) Differential-difference equation. For $u \geq 1$,

$$
u \rho^{\prime}(u)=-\rho(u-1) \text {. }
$$

\footnotetext{
${ }^{30} p^{e}|| n$ means $p^{e} \mid n$ and $p^{e+1} \nmid n$.
} 
The Dickman function is alternatively characterized as the solution on the real line to the integral equation

$$
u \rho(u)=\int_{0}^{1} \rho(u-t) d t
$$

(for $u \rho(u)$ ) with initial condition as above, extended to require that $\rho(u)=0$ for all $u<0$, as shown in de Bruijn [50, (2.1)]. Another explicit form for $\rho(u)$ is the iterated integral form

$$
\rho(u)=1+\sum_{k=1}^{\lfloor u\rfloor} \frac{(-1)^{k}}{k !} \int_{\substack{t_{1}+\cdots+t_{k} \leq u \\ t_{1}, \ldots, t_{k} \geq 1}} \frac{d t_{1}}{t_{1}} \frac{d t_{2}}{t_{2}} \cdots \frac{d t_{k}}{t_{k}},
$$

which also occurs in connection with random permutations; see (3.10.2) ff.

It is known that for $u \geq 1$, the Dickman function $\rho(u)$ is a strictly decreasing positive function that decreases rapidly, satisfying

$$
\rho(u) \leq \frac{1}{\Gamma(u+1)}
$$

see Norton [240, Lemma 4.7]. The key relation of the Dickman function to Euler's constant is that it has total mass $e^{\gamma}$; see Theorem 3.5.1 below.

In 1951 de Bruijn [50] gave an exact expression for the Dickman function as a contour integral,

$$
\rho(u)=\frac{1}{2 \pi i} \int_{-i \infty}^{i \infty} \exp \left(\gamma+\int_{0}^{z} \frac{e^{s}-1}{s} d s\right) e^{-u z} d z(u>0) .
$$

One may also consider the one-sided Laplace transform of the Dickman function, which we denote

$$
\hat{\rho}(s):=\int_{0}^{\infty} \rho(u) e^{-u s} d u
$$

following Tenenbaum [293, III.5.4]. This integral converges absolutely for all $s \in \mathbb{C}$ and defines $\hat{\rho}(s)$ as an entire function. To evaluate it, recall that the complementary exponential integral

$$
\operatorname{Ein}(z):=\int_{0}^{z} \frac{1-e^{-t}}{t} d t=\sum_{n=1}^{\infty}(-1)^{n-1} \frac{1}{n \cdot n !} z^{n},
$$

is an entire function of $z$.

Theorem 3.5.1 (de Bruijn 1951, van Lint and Richert 1964). The one-sided Laplace transform $\hat{\rho}(s)$ of the Dickman function $\rho(u)$ is an entire function, given by

$$
\hat{\rho}(s)=e^{\gamma-\operatorname{Ein}(s)},
$$

in which $\operatorname{Ein}(s)$ is the complementary exponential integral. In particular, the total mass of the Dickman function is

$$
\hat{\rho}(0)=\int_{0}^{\infty} \rho(u) d u=e^{\gamma}
$$

Proof. Inside de Bruijn's formula (3.5.4) there appears the integral

$$
I(s):=\int_{0}^{s} \frac{e^{t}-1}{t} d t=-\operatorname{Ein}(-s) .
$$


This formula determines the Fourier transform of the Dickman function to be $e^{\gamma+I(-i t)}$. The change of variable $s=-i t$ yields the the one-sided Laplace transform of $\rho(u)$ above. The total mass identity (3.5.7) was noted in 1964 in van Lint and Richert 204. Moreover they noted that as $u \rightarrow \infty$,

$$
\int_{0}^{u} \rho(t) d t=e^{\gamma}+O\left(e^{-u}\right)
$$

A direct approach to the determination of the Laplace transform given in (3.5.6) was given by Tenenbaum [293, III.5.4, Theorem 7] in 1995. His proof uses a lemma showing that for all $s \in \mathbb{C} \backslash(-\infty, 0]$ one has

$$
-I(-s)=\operatorname{Ein}(s)=\gamma+\log s+J(s),
$$

with

$$
J(s):=\int_{0}^{\infty} \frac{e^{-s-t}}{s+t} d t .
$$

Tenenbaum sketches a direct arithmetic proof of the identity (3.5.7) in 293, III.5 Exercise 2, p. 392]; in this argument the quantity $e^{\gamma}$ is derived using Mertens's product theorem. We note the identity

$$
J(s)=\mathrm{E}_{1}(s),
$$

in which $\mathrm{E}_{1}(s):=\int_{s}^{\infty} \frac{e^{-t}}{t} d t$ is the principal exponential integral. The function $\mathrm{E}_{1}(s)$ appeared in connection with Euler's divergent series in Section 2.5] see (2.5.14).

In 1973 Chamayou [56] found a probabilistic interpretation of the Dickman function $\rho(x)$ which is suitable for computing it by Monte Carlo simulation.

Theorem 3.5.2 (Chamayou 1973). Let $X_{1}, X_{2}, X_{3}, \ldots$ be a sequence of independent identically distributed random variables with uniform distribution on $[0,1]$. Set

$$
P(u):=\operatorname{Prob}\left[X_{1}+X_{1} X_{2}+X_{1} X_{2} X_{3}+\cdots \leq u \text {. }\right]
$$

This function is well defined for $u \geq 0$, and its derivative $P^{\prime}(u)$ satisfies

$$
\rho(u)=e^{\gamma} P^{\prime}(u) .
$$

A discrete identity relating the Dickman function and $e^{\gamma}$ was noted by Knuth and Trabb-Pardo [180, which complements the continuous identity (3.5.7).

Theorem 3.5.3 (Knuth and Trabb-Pardo 1976). For $0 \leq x \leq 1$ the Dickman function satisfies the identity

$$
x+\sum_{n=1}^{\infty}(x+n) \rho(x+n)=e^{\gamma} .
$$

In particular, taking $x=0$,

$$
\rho(1)+2 \rho(2)+3 \rho(3)+\cdots=e^{\gamma} .
$$

Proof. The identity (3.5.10) immediately follows from (3.5.7), using the integral equation (3.5.2) for $u \rho(u)$.

We conclude this section by using formulas above to deduce a curious identity relating a special value of this Laplace transform to the Euler-Gompertz constant $\delta$ given in Section 2.4; compare also Theorem 3.16.2. 
Theorem 3.5.4. The Laplace transform $\hat{\rho}(s)$ of the Dickman function $\rho(u)$ is given at $s=1$ by

$$
\hat{\rho}(1):=\int_{0}^{\infty} \rho(u) e^{-u} d u=e^{-\frac{\delta}{e}},
$$

in which $\delta:=\int_{0}^{1} \frac{d v}{1-\log v}$ is the Euler-Gompertz constant,

Proof. By definition $\hat{\rho}(1)=\int_{0}^{\infty} \rho(u) e^{-u} d u$. We start from Hardy's formula (2.5.11) for the Euler-Gompertz constant:

$$
\delta=s(1)=\int_{0}^{\infty} \frac{e^{-w}}{1+w} d w .
$$

Comparison with (3.5.9) then yields

$$
\mathrm{E}_{1}(1)=J(1)=\int_{0}^{\infty} \frac{e^{-1-t}}{1+t} d t=\frac{\delta}{e} .
$$

Now (3.5.8) evaluated at $s=1$ gives

$$
\operatorname{Ein}(1)=\gamma+\mathrm{E}_{1}(1)=\gamma+\frac{\delta}{e}
$$

which can be rewritten using (3.5.5) as

$$
\int_{0}^{1} \frac{1-e^{-t}}{t} d t=\gamma+\frac{\delta}{e}
$$

We conclude from Theorem 3.5.1 that

$$
\hat{\rho}(1)=e^{\gamma-\operatorname{Ein}(1)}=e^{-\frac{\delta}{e}},
$$

which is the assertion.

Hildebrand and Tenenbaum [165] and Granville [140 give detailed surveys including estimates for $\Psi(x, y)$, a field sometimes called psixyology; see Moree [224]. It has recently been uncovered that Ramanujan had results on the Dickman function in his "Lost Notebook", prior to the work of Dickman; see Moree [225, Section 2.4].

3.6. Euler's constant and sieve methods: the Buchstab function. Euler's constant also arises in sieve methods in number theory in the complementary problem where one removes integers having some small prime factor. Let $\Phi(x, y)$ count the number of integers $n \leq x$ having no prime factor $p \leq y$. In 1937 Buchstab 52 ] (see also [53]) established for $u>1$ an asymptotic formula

$$
\Phi\left(x, x^{\frac{1}{u}}\right) \sim u \omega(u) \frac{x}{\log x},
$$

for a certain function $\omega(u)$ named by de Bruijn [49] the Buchstab function.

The Buchstab function is defined for $u \geq 1$ by the properties:

(1) Initial conditions. For $1 \leq u \leq 2$, it satisfies

$$
\omega(u)=\frac{1}{u} .
$$

(2) Differential-difference equation. For $u \geq 2$,

$$
(u \omega(u))^{\prime}=\omega(u-1) .
$$


This function is alternatively characterized as the solution on the real line to the integral equation

$$
u \omega(u)=1+\int_{1}^{u-1} \omega(t) d t
$$

as shown in de Bruijn [49, (2.1)], who determined properties of this function. Another explicit formula for this function, valid for $u>2$, is

$$
\begin{aligned}
u \omega(u)=1+\sum_{2 \leq k \leq\lfloor u\rfloor} \frac{1}{k !} \int_{\substack{1 / u \leq 1-\left(y_{1}+y_{2}+\cdots+y_{k-1}\right) \leq 1 \\
1 / u y_{1}+1}} & \times \frac{d y_{1} d y_{2} \cdots d y_{k-1}}{y_{1} y_{2} \cdots y_{k-1}\left(1-\left(y_{1}+y_{2}+\cdots+y_{k-1}\right)\right)} .
\end{aligned}
$$

It is known that the function $\omega(u)$ is oscillatory and satisfies

$$
\lim _{u \rightarrow \infty} \omega(u)=e^{-\gamma} \text {. }
$$

The convergence to $e^{-\gamma}$ is extremely rapid, and satisfies the estimate

$$
\omega(u)=e^{-\gamma}+O\left(u^{-u / 2}\right) \text { for } u \in[2, \infty) .
$$

Proofs of these results can be found in Montgomery and Vaughan [223, Section 7.2] and in Tenenbaum [293, Chapter III.6].

The convergence behavior of the Buchstab function to $e^{\gamma}$ as $x \rightarrow \infty$ played a crucial role in the groundbreaking 1985 work of Helmut Maier [210] showing the existence of large fluctuations in the density of primes in short intervals; this work was mentioned at the end of Section 3.3. Maier [210, Lemma 4] showed that $\omega(u)-e^{-\gamma}$ changes sign at least once on each interval $[a-1, a]$ for any $a \geq 2$. In 1990 Cheer and Goldston [57] showed there are at most two sign changes and at most two critical points on an interval $[a-1, a]$, and Hildebrand [164] showed that the spacing between such sign changes approaches 1 as $u \rightarrow \infty$.

A consequence of the analysis of de Bruijn [49] is that the constant $e^{-\gamma}$ appears as a universal limiting constant for sieve methods, for a wide range of sieve cutoff functions $y=y(x)$ growing neither too slow nor too fast compared to $x$.

Theorem 3.6.1 (de Bruijn 1950). Suppose that $y=y(x)$ depends on $x$ in such $a$ way that both $y \rightarrow \infty$ and $\frac{\log y}{\log x} \rightarrow 0$ hold as $x \rightarrow \infty$. Under these conditions we have

$$
\Phi(x, y) \sim e^{-\gamma} \frac{x}{\log y} .
$$

Proof. This result can be deduced from de Bruijn [49, (1.7)]. This asymptotic formula also follows as a corollary of very general estimate ([293, III.6.2, Theorem 3]) valid uniformly on the region $x \geq y \geq 2$, which states

$$
\Phi(x, y)=\omega\left(\frac{\log x}{\log y}\right) \frac{x}{\log y}-\frac{y}{\log y}+O\left(\frac{x}{(\log y)^{2}}\right) .
$$

The formula (3.6.3) follows using the estimate (3.6.2) for $\omega(u)$, which applies since the hypotheses $x / y \rightarrow \infty$ when $x \rightarrow \infty$ imply $\frac{\log x}{\log y} \rightarrow \infty$.

The one-sided Laplace transform of the Buchstab function is given as

$$
\hat{\omega}(s):=\int_{0}^{\infty} e^{-s u} \omega(u) d u,
$$


where we make the convention that $\omega(u)=0$, for $0 \leq u<1$. Under this convention this Laplace transform has a simple relation with the Laplace transform of the Dickman function.

Theorem 3.6.2 (Tenenbaum 1995). The one-sided Laplace transform $\hat{\omega}(s)$ defined for $\operatorname{Re}(s)>0$ by (3.6.4), extends to a meromorphic function on $\mathbb{C}$, given explicitly by the form

$$
1+\hat{\omega}(s)=\frac{1}{s \hat{\rho}(s)} \quad(s \neq 0) .
$$

When $s$ is not real and negative,

$$
1+\hat{\omega}(s)=e^{J(s)},
$$

with $J(s)=\int_{0}^{\infty} \frac{e^{-s-t}}{s+t} d t=E_{1}(s)$.

Proof. The Laplace transforms individually have been evaluated many times in the sieve method literature; see Wheeler [316. Theorem 2, ff], who also gives references back to the 1960s. One has explicitly that

$$
\hat{\omega}(s)=\frac{1}{s} \exp (-\operatorname{Ein}(s)),
$$

in terms of the complementary exponential integral. The identity 3.6.5 connecting them seems to be first explicitly stated in Tenenbaum [293, III.6, Theorem 5].

Combining this result with Theorem 3.5.1 gives

$$
\hat{\omega}(s) \sim \frac{e^{-\gamma}}{s}, \quad \text { as } s \rightarrow 0 .
$$

We also obtain using (3.6.6), (3.6.7) along with (2.5.16) that

$$
\hat{\omega}(1):=\int_{0}^{\infty} \omega(u) e^{-u} d u=e^{-\gamma} \exp \left(-\mathrm{E}_{1}(1)\right)=e^{-\gamma} e^{-\delta / e},
$$

where $\delta$ is the Euler-Gompertz constant.

Wheeler [316, Theorem 2] also observes that the solutions to the differentialdifference equations for the Dickman function and the Buchstab function are distinguished from those for general initial conditions by the special property that their Laplace transforms analytically continue to entire (resp. meromorphic) functions of $s \in \mathbb{C}$.

For a retrospective look at the work of N. G. de Bruijn on $\rho(x)$ and $\Psi(x, y)$ in Section 3.6. and of $\omega(x)$ and $\Phi(x, y)$ in this section, see Moree 225].

3.7. Euler's constant and the Riemann hypothesis. Several formulations of the Riemann hypothesis can be given in terms of Euler's constant. Here we describe some that involve the approach towards the extremal behaviors of Euler's totient function $\phi(n)$ and the sum of divisors function $\sigma(n)$ given in Section 3.4. The Riemann hypothesis is also related, in another way, to generalized Euler's constants considered in Section 3.8 .

In 1981 J.-L. Nicolas ([236], 237]) proved that the Riemann hypothesis is encoded in the property that the Euler totient function values $\phi\left(n_{k}\right)$ for the extremal numbers $n_{k}=p_{1} p_{2} \cdots p_{k}$ given in Landau's theorem approach their limiting value from one side only. Nicolas stated his result using the inverse of Landau's quantity, as follows. 
Theorem 3.7.1 (Nicolas 1981). The Riemann hypothesis holds if and only if all the primorial numbers $N_{k}=p_{1} p_{2} \cdots p_{k}$ with $k \geq 2$ satisfy

$$
\frac{N_{k}}{\phi\left(N_{k}\right) \log \log N_{k}}>e^{\gamma} \text {. }
$$

If the Riemann hypothesis is false, then these inequalities will be true for infinitely many primorial numbers $N_{k}$ and false for infinitely many $N_{k}$.

This inequality is equivalent to the statement that the Riemann hypothesis implies that all the primorial numbers $N_{k}$ for $k \geq 2$ undershoot the asymptotic lower bound $e^{-\gamma}$ in Landau's theorem (Theorem 3.4.2). As an example, $\phi(2 \cdot 3 \cdot 5 \cdot 7)=$ $\phi(210)=48$ and

$$
\frac{\phi\left(n_{4}\right) \log \log n_{4}}{n_{4}}=\frac{\phi(210) \log \log 210}{210}=0.38321 \cdots<e^{-\gamma}=0.56145 \cdots .
$$

For most $n$ one will have $\frac{\phi(n) \log \log n}{n}>e^{-\gamma}$, and only a very thin subset of $n$ will be close to this bound. For example taking $n=p_{k}$, a single prime, one sees that as $k \rightarrow \infty$, this ratio goes to $+\infty$.

Very recently Nicolas 238] obtained a refined encoding of the Riemann hypothesis in terms of a sharper asymptotic for small values of the Euler $\phi$-function, in which both $\gamma$ and $e^{\gamma}$ appear.

Theorem 3.7.2 (Nicolas 2012). For each integer $n \geq 2$ set

$$
c(n):=\left(\frac{n}{\phi(n)}-e^{\gamma} \log \log n\right) \sqrt{\log n} .
$$

Then the Riemann hypothesis is equivalent to the statement that

$$
\limsup _{n \rightarrow \infty} c(n)=e^{\gamma}(4+\gamma-\log (4 \pi)) .
$$

Proof. The assertion that the Riemann hypothesis implies 3.7.1 holds is 238, Theorem 1.1], and the converse assertion is [238, Corollary 1.1]. Here the constant

$$
e^{\gamma}(4+\gamma-\log (4 \pi))=e^{\gamma}(2+\beta)=3.6444150964 \cdots,
$$

in which

$$
\beta=\sum_{\rho} \frac{1}{\rho(1-\rho)}=2+\gamma-\log (4 \pi)=0.0461914179 \cdots,
$$

where in the sum $\rho$ runs over the nonreal zeros of the zeta function, counted with multiplicity. For the converse direction, he shows that if the Riemann hypothesis fails, then

$$
\limsup _{n \rightarrow \infty} c(n)=+\infty .
$$

Note that $\liminf _{n \rightarrow \infty} c(n)=-\infty$ holds unconditionally.

In 1984 G. Robin [265] showed that for the sum of divisors function $\sigma(n)$ the Riemann hypothesis is also encoded as a one-sided approach to the limit in Gronwall's theorem (Theorem 3.4.3).

Theorem 3.7.3 (Robin 1984). The Riemann hypothesis holds if and only if the inequalities

$$
\frac{\sigma(n)}{n \log \log n}<e^{\gamma}
$$


are valid for all $n \geq 5041$. If the Riemann hypothesis is false, then this inequality will be true for infinitely many $n$ and false for infinitely many $n$.

Robin's result says that the Riemann hypothesis is equivalent to the limiting value $e^{\gamma}$ being approached from below, for all sufficiently large $n$. The inequality (3.7.2) fails to hold for a few small $n$, the largest known exception being $n=5040$. Here the extremal numbers giving record values for $f(n)=\frac{\sigma(n)}{n}$ will have a different form than that in Nicolas's theorem. It is known that infinitely many of the extremal numbers will be colossally abundant numbers, as defined by Alaoglu and Erdős 3] in 1944. These are numbers $n$ such that there is some $\epsilon>0$ such that

$$
\frac{\sigma(n)}{n^{1+\epsilon}} \geq \frac{\sigma(k)}{k^{1+\epsilon}}, \text { for } 1 \leq k<n .
$$

Alaoglu and Erdős showed that the "generic" colossally abundant number is a product of powers of small primes having a sequence of exponents by a parameter $\epsilon>0$ as

$$
n=n(\epsilon):=\prod_{p} p^{a_{p}(\epsilon)}
$$

in which

$$
a_{p}(\epsilon):=\left\lfloor\frac{\log \left(p^{1+\epsilon}-1\right)-\log \left(p^{\epsilon}-1\right)}{\log p}\right\rfloor-1 .
$$

For fixed $\epsilon$ the sequence of exponents $a_{p}(\epsilon)$ is nonincreasing as $p$ increases and becomes 0 for large $p$. However for fixed $p$ the exponent $a_{p}(\epsilon)$ increases as $\epsilon$ decreases towards 0 , so that $n(\epsilon) \rightarrow \infty$ as $\epsilon \rightarrow 0^{+}$. Colossally abundant and related numbers had actually been studied by Ramanujan [253] in 1915, but the relevant part of his paper was suppressed by the London Mathematical Society to save expense. The suppressed part of the manuscript was recovered in his "Lost Notebook", and later published in 1997 (255]).

In 2002 this author (191]), starting from Robin's result, obtained the following elementary criterion for the Riemann hypothesis, involving the sum of divisors function $\sigma(n)$ and the harmonic numbers $H_{n}$.

Theorem 3.7.4. The Riemann hypothesis is equivalent to the assertion that for each $n \geq 1$ the inequality

$$
\sigma(n) \leq e^{H_{n}} \log H_{n}+H_{n}
$$

is valid. Assuming the Riemann hypothesis, equality holds if and only if $n=1$.

The additive term $H_{n}$ is included in this formula for elegance, as it makes the result hold for all $n \geq 1$ (assuming the Riemann hypothesis), rather than be valid for $n \geq 5041$, as in Robin's theorem 3.7.3. The converse direction of this result requires additional proof, which is accomplished in [191] using certain asymptotic estimates obtained in Robin's paper [265. It shows that if the Riemann hypothesis is false, then the inequalities (3.7.4) will fail to hold for infinitely many positive integers $n$.

3.8. Generalized Euler constants and the Riemann hypothesis. In 1961 W. Briggs [47] introduced the notion of an Euler constant associated to an arithmetic progression of integers. This notion was studied in detail by D. H. Lehmer 
[201] in 1975. For the arithmetic progression $h(\bmod k)$ with $0 \leq h<k$, we set

$$
\gamma(h, k):=\lim _{x \rightarrow \infty}\left(\sum_{\substack{0<n \leq x \\ n \equiv h(\bmod k)}} \frac{1}{n}-\frac{\log x}{k}\right) .
$$

These constants were later termed Euler-Lehmer constants by Murty and Saradha [228. Here one has $\gamma(0,1)=\gamma, \gamma(1,2)=\frac{1}{2}(\gamma+\log 2)$ and $\gamma(2,4)=\frac{1}{4} \gamma$, where $\gamma$ is Euler's constant. It suffices to study the constants where $\operatorname{gcd}(h, k)=1$, other cases reduce to these by dividing out the greatest common factor.

We next define generalized Euler constants $\gamma(\Omega)$ associated to a finite set of primes $\Omega=\left\{p_{i_{1}}, \ldots, p_{i_{k}}\right\}$, possibly empty. These constants were introduced by Diamond and Ford [71] in 2008. To specify them, we first define a zeta function $Z_{\Omega}(s)$ by

$$
Z_{\Omega}(s):=\left(\prod_{p \in \Omega}\left(1-\frac{1}{p^{s}}\right)\right) \zeta(s)=\sum_{n=1}^{\infty} \mathbf{1}_{\Omega}(n) n^{-s}=\sum_{g c d\left(n, P_{\Omega}\right)=1} n^{-s},
$$

where we set

$$
\mathbf{1}_{\Omega}(j)= \begin{cases}1 & \text { if }\left(n, P_{\Omega}\right)=1 \\ 0 & \text { otherwise }\end{cases}
$$

in which

$$
P_{\Omega}:=\prod_{p \in \Omega} p
$$

The function $Z_{\Omega}(s)$ defines a meromorphic function on the entire plane which has a simple pole at $s=1$ with residue

$$
D(\Omega):=\prod_{p \in \Omega}\left(1-\frac{1}{p}\right) .
$$

The generalized Euler constant $\gamma(\Omega)$ associated to $\Omega$ is the constant term in the Laurent expansion of $Z_{\Omega}(s)$ around $s=1$, namely

$$
Z_{\Omega}(s)=\frac{D(\Omega)}{s-1}+\gamma(\Omega)+\sum_{j=1}^{\infty} \gamma_{k}(\Omega)(s-1)^{k} .
$$

In the case that $\Omega=\emptyset$ is the empty set, this function $Z_{\Omega}(s)$ is exactly the Riemann zeta function, and $\gamma(\emptyset)=\gamma$ by Theorem 3.2.1. These numbers $\gamma(\Omega)$ generalize the characterization of Euler's constant in (3.2.2) in the sense that

$$
\gamma(\Omega)=\lim _{n \rightarrow \infty}\left(\sum_{j=1}^{n} \frac{\mathbf{1}_{\Omega}(j)}{j}-D(\Omega) \log n\right) .
$$

The constants $\gamma(\Omega)$ are easily shown to be finite sums of the Euler-Lehmer constants

$$
\gamma(\Omega)=\sum_{\substack{1 \leq h<P_{\mathcal{P}} \\ g c d\left(h, P_{\Omega}\right)=1}} \gamma\left(h, P_{\Omega}\right) .
$$

Diamond and Ford [71, Theorem 1] show that these generalized Euler constants are related to Euler's constant in a second way which involves the constant $e^{-\gamma}$ rather than $\gamma$. Let $\Omega_{r}$ denote the set of the first $r$ primes and set $\gamma_{r}:=\gamma\left(\Omega_{r}\right)$. 
Theorem 3.8.1 (Diamond and Ford 2008). Let $\Gamma=\inf \{\gamma(\mathcal{P}):$ all finite $\mathcal{P}\}$. Then:

(1) The values of $\gamma(\mathcal{P})$ are dense in $[\Gamma, \infty)$.

(2) The constant $\Gamma$ satisfies

$$
0.56 \leq \Gamma \leq e^{-\gamma}
$$

If $\Gamma<e^{-\gamma}$, then the value $\Gamma$ is attained at some $\gamma_{r}$.

Proof. (1) This is shown as [71, Proposition 2].

(2) Diamond and Ford show that each $\gamma(\mathcal{P}) \geq \gamma_{r}$ for some $1 \leq r<|\mathcal{P}|$. This implies

$$
\Gamma=\inf \left\{\gamma_{r}: r \geq 1\right\} .
$$

Merten's formulas for sums and products yield the asymptotic formula

$$
\gamma_{r} \sim e^{-\gamma} \text { as } r \rightarrow \infty
$$

which by 3.8.3) implies that $\Gamma \leq e^{-\gamma}$. If $\Gamma<e^{-\gamma}$, then it must be attained by one of the $\gamma_{r}$, For there must be at least one $\gamma_{r_{1}}<e^{-\gamma}$, and by (3.8.4) there are only finitely many $\gamma_{r} \leq \gamma_{r_{1}}$ and one of these must attain the infimum by the bound above. Diamond and Ford established the lower bound $\gamma_{r} \geq 0.56$ for all $r \geq 1$.

Diamond and Ford [71, Theorem 2] also show that the behavior of the quantities $\gamma(\Omega)$ is complicated as the set $\Omega$ increases, in the sense that $\gamma_{r}$ is not a monotone function of $r$, with $\gamma_{r+1}>\gamma_{r}$ and $\gamma_{r+1}<\gamma_{r}$ each occurring infinitely often. Nevertheless Diamond and Ford [71, Theorems 3, 4] obtain the following elegant reformulation of the Riemann hypothesis.

Theorem 3.8.2 (Diamond and Ford 2008). The Riemann hypothesis is equivalent to either one of the following assertions.

(1) The infimum $\Gamma$ of $\gamma(\mathcal{P})$ satisfies

$$
\Gamma=e^{-\gamma} .
$$

(2) For every finite set $\Omega$ of primes

$$
\gamma(\Omega)>e^{-\gamma}
$$

Here (2) implies (1), and (2) says that if the Riemann hypothesis is true, then (3.8.5) holds for all $\Omega$ and the infimum $\Gamma=e^{-\gamma}$ is not attained, while if it does not hold, then by Theorem 3.8.1 (1) the reverse inequality holds for infinitely many $\Omega$, and the infimum is attained. Theorem 3.8.2 seems remarkable: on taking $\mathcal{P}=\emptyset$ to be the empty set, it already requires, for the truth of the Riemann hypothesis, that

$$
\gamma=0.57721 \cdots>e^{-\gamma}=0.56145 \cdots .
$$

In consequence the unique real root $x_{0} \approx 0.56714$ of the equation $x_{0}=e^{-x_{0}}$ necessarily satisfies $\gamma>x_{0}>e^{-\gamma}$.

Results on the transcendence of Euler-Lehmer constants $\gamma(h, k)$ and generalized Euler constants $\gamma(\Omega)$ have recently been established; see Section 3.16.

Finally we note that one may define more generally higher order Euler-Lehmer constants. For $j \geq 1$ Dilcher [74] defines for $h \geq 1$, and $0<k \leq h$, the constants

$$
\gamma_{j}(h, k):=\lim _{x \rightarrow \infty}\left(\sum_{\substack{0<n \leq x \\ n \equiv h(\bmod k)}} \frac{(\log n)^{j}}{n}-\frac{(\log x)^{j+1}}{k(j+1)}\right) .
$$


Here $j$ is the order, with $\gamma_{1}(h, k)=\gamma(h, k)$ being the Euler-Lehmer constants, and $\gamma_{j}(1,1)=\gamma_{j}$ is the $j$ th Stieltjes constant (see Theorem 3.2.1). Dilcher relates these constants to values of derivatives of the digamma function at rational points and also to derivatives of Dirichlet $L$-functions evaluated at $s=1$.

3.9. Euler's constant and extreme values of $\zeta(1+i t)$ and $L\left(1, \chi_{-d}\right)$. Some of the deepest questions in number theory concern the distribution of values of the Riemann zeta function and its generalizations, Dirichlet $L$-functions. In this section we consider such value distributions at the special point $s=1$ and on the vertical line $\operatorname{Re}(s)=1$. Here Euler's constant appears in connection with the size of extreme values of $\zeta(1+i t)$ as $t \rightarrow \infty$. It also appears in the same guise in connection with extreme values of the Dirichlet $L$-function $L\left(s, \chi_{-d}\right)$ at the point $s=1$ where $\chi_{-d}$ is the real primitive Dirichlet character associated to the quadratic field $\mathbb{Q}(\sqrt{-d})$, and we let $d \rightarrow \infty$. (The quantity $-d$ denotes a fundamental discriminant of an imaginary quadratic field, which is necessarily squarefree away from the prime 2.) In both cases these connections were first made by J. E. Littlewood. Some of his results are conditional on the Riemann hypothesis or the generalized Riemann hypothesis, while others are unconditional.

In these results Euler's constant occurs by way of Merten's theorem (Theorem 3.3.2). The basic idea, in the context of large values of $|\zeta(1+i t)|$, is that large values will occur (only) when a suitable finite truncation of its Euler product representation is large, and this in turn will occur when the phases of the individual terms in the product line up properly. Mertens's theorem is then used in estimating the size of this Euler product.

We first consider extreme values of $\zeta(1+i t)$. To place results in context, it is known unconditionally that $|\zeta(1+i t)|=O(\log |t|)$ and that $\frac{1}{\zeta(1+i t) \mid}=O(\log |t|)$ as $|t| \rightarrow \infty$. In 1926 Littlewood determined, assuming the Riemann hypothesis, the correct maximal order of growth of the Riemann zeta function on the line $\operatorname{Re}(s)=1$, and in 1928 he obtained a corresponding bound for $\frac{1}{\zeta(1+i t)}$.

Theorem 3.9.1 (Littlewood 1926, 1928). Assume the Riemann hypothesis. Then:

(1) The maximal order of $|\zeta(1+i t)|$ is at most $\log \log t$, with

$$
\limsup _{t \rightarrow \infty} \frac{|\zeta(1+i t)|}{\log \log t} \leq 2 e^{\gamma} .
$$

(2) The maximal order of $\frac{1}{|\zeta(1+i t)|}$ is at most $\log \log t$, with

$$
\limsup _{t \rightarrow \infty} \frac{1}{|\zeta(1+i t)| \log \log t} \leq \frac{2}{\zeta(2)} e^{\gamma} .
$$

Proof. The bound (1) is 205, Theorem 7] and the bound (2) is [206, Theorem $1]$.

Littlewood also established unconditionally a lower bound for the quantity in (1) and, conditionally on the Riemann hypothesis, a lower bound for that in (2), both of which matched the bounds above up to a factor of 2. In 1949 a method of Chowla 58 made this lower bound for the quantity in (2) unconditional. These combined results are as follows.

Theorem 3.9.2 (Littlewood 1926, Titchmarsh 1933). The following bounds hold unconditionally. 
(1) The maximal order of $|\zeta(1+i t)|$ is at least $\log \log t$, with

$$
\limsup _{t \rightarrow \infty} \frac{|\zeta(1+i t)|}{\log \log t} \geq e^{\gamma}
$$

(2) The maximal order of $\frac{1}{|\zeta(1+i t)|}$ is at least $\log \log t$, with

$$
\limsup _{t \rightarrow \infty} \frac{1}{|\zeta(1+i t)| \log \log t} \geq \frac{1}{\zeta(2)} e^{\gamma} .
$$

Proof. The bound (1) is Theorem 8 of [205]. The lower bound (2) was proved, assuming the Riemann hypothesis, in 1928 by Littlewood [206]. An unconditional proof was later given by Titchmarsh [299; see also [300, Theorem 8.9(B)].

Littlewood's proof of (1) used Diophantine approximation properties of the values $\log p$ for primes $p$, namely the fact that they are linearly independent over the rationals. This guarantees that there exist suitable values of $t$ where the all terms in the Euler product $\prod_{p \leq X}\left(1-\frac{1}{p^{1+i t}}\right)^{-1}$ have most phases $t \log p$ near $0(\bmod 2 \pi)$ for $p \leq X$.

Littlewood was struck by the fact that the unconditional lower bound for $\limsup \operatorname{su}_{t \rightarrow \infty} \frac{|\zeta(1+i t)|}{\log \log t}$ in Theorem $3.9 .2(1)$ and the conditional upper bound for it given in Theorem 3.9.1(1) differ by the simple multiplicative factor 2. He discusses this fact at length in [206], 207]. The Riemann hypothesis is not sufficient to predict the exact value! It seems that more subtle properties of the Riemann zeta function, perhaps related to Diophantine approximation properties of prime numbers or of the imaginary parts of zeta zeros, will play a role in determining the exact constant. Littlewood favored the right answer as being the lower bound in Theorem 3.9.2 saying ([207, p. 359]):

The results involving $c\left[=e^{\gamma}\right]$ are evidently final except for a certain factor 2. I showed also that on a certain further hypothesis (which there is, perhaps, no good reason for believing) this factor 2 disappears.

In the fullness of time this statement has been elevated to a conjecture attributed to Littlewood.

There is now relatively strong evidence favoring the conjecture that the lower bound (1) in Theorem 3.9.2 should be equality. In 2006 Granville and Soundararajan [143] obtained a bound for the frequency of occurrence of extreme values of $|\zeta(1+i t)|$, considering the quantity

$$
\Phi_{T}(\tau):=\frac{1}{T} \operatorname{meas}\left\{t \in[T, 2 T]:|\zeta(1+i t)|>e^{\gamma} \tau\right\},
$$

where meas denotes Lebesgue measure. They established a result [143, Theorem 1] showing that for all sufficiently large $T$, uniformly in the range $1 \ll \tau \leq \log \log T+$ 20 , there holds

$$
\Phi_{T}(\tau)=\exp \left(-\frac{2 e^{\tau-C-1}}{\tau}\left(1+O\left(\frac{1}{\sqrt{\tau}}+\left(\frac{e^{\tau}}{\log T}\right)^{1 / 2}\right)\right)\right),
$$

where $C$ is a positive constant. An extension of these ideas established the following result ([143, Theorem 2]). 
Theorem 3.9.3 (Granville and Soundararajan 2006). For all sufficiently large T, the measure of the set of all points $t$ in $[T, 2 T]$ having

$$
|\zeta(1+i t)| \geq e^{\gamma}(\log \log T+\log \log \log T-\log \log \log \log T-\log A+O(1))
$$

is at least $T^{1-\frac{1}{A}}$, uniformly for $A \geq 10$.

They observe that if the estimate (3.9.1) remained valid without restriction on the range of $\tau$, this would correspond to the following stronger assertion.

Conjecture 3.9.4 (Granville and Soundararajan 2006). There is a constant $C_{1}$ such that for $T \geq 10$,

$$
\max _{T \leq t \leq 2 T}|\zeta(1+i t)|=e^{\gamma}\left(\log \log T+\log \log \log T+C_{1}+o(1)\right) .
$$

This conjecture implies that the lower bound (1) in Theorem 3.9 .2 would be an equality.

We next consider the distribution of extreme values of the Dirichlet $L$-functions $L\left(s, \chi_{-d}\right)$ at $s=1$, for real primitive characters $\chi_{-d}(n)=\left(\frac{-d}{n}\right)$, where $-d$ is the discriminant of an imaginary quadratic field $\mathbb{Q}(\sqrt{-d})$. The Dirichlet $L$-function

$$
L\left(s, \chi_{-d}\right)=\sum_{n=1}^{\infty}\left(\frac{-d}{n}\right) n^{-s},
$$

is a relative of the Riemann zeta function, having an Euler product and a functional equation. Dirichlet's class number formula states that for a fundamental discriminant $-d$ with $d>0$,

$$
L\left(1, \chi_{-d}\right)=\frac{2 \pi h(-d)}{w_{-d} \sqrt{d}}
$$

where $h(-d)$ is the order of the ideal class group in $\mathbb{Q}(\sqrt{-d})$ and $w_{-d}$ is the number of units in $\mathbb{Q}(\sqrt{-d})$ so that $w_{-d}=2$ for $d>4, w_{-3}=6$, and $w_{-4}=4$. Thus the size of $L\left(1, \chi_{-d}\right)$ encodes information on the size of the class group, and the formula shows that $L\left(1, \chi_{-d}\right)>0$.

Theorem 3.9.5 (Littlewood 1928). Assume the generalized Riemann hypothesis for all real primitive characters $L\left(s, \chi_{-d}\right)$, where $-d$ is the discriminant of an imaginary quadratic field $\mathbb{Q}(\sqrt{-d})$. Then:

(1) The maximal order of $L\left(1, \chi_{-d}\right)$ is at most $\log \log d$, with

$$
\limsup _{d \rightarrow \infty} \frac{L\left(1, \chi_{-d}\right)}{\log \log d} \leq 2 e^{\gamma} .
$$

(2) The maximal order of $\frac{1}{L\left(1, \chi_{-d}\right)}$ is at most $\log \log d$, with

$$
\limsup _{d \rightarrow \infty} \frac{1}{L\left(1, \chi_{-d}\right) \log \log d} \leq \frac{2}{\zeta(2)} e^{\gamma} .
$$

Proof. The bounds (1) and (2) are the content of [207, Theorem 1].

There are corresponding unconditional lower bounds that differ from these by a factor of 2, as noted by Littlewood in 1928 for the bound (1) and completed by a result of Chowla [58] for the bound (2). 
Theorem 3.9.6 (Littlewood 1928, Chowla 1949). The following bounds hold unconditionally as $d \rightarrow \infty$ with $-d$ a fundamental discriminant.

(1) The maximal order of $L\left(1, \chi_{-d}\right)$ is at least $\log \log d$, with

$$
\limsup _{d \rightarrow \infty} \frac{L\left(1, \chi_{-d}\right)}{\log \log d} \geq e^{\gamma}
$$

(2) The maximal order of $\frac{1}{L\left(1, \chi_{-d}\right)}$ is at least $\log \log d$, with

$$
\limsup _{d \rightarrow \infty} \frac{1}{L\left(1, \chi_{-d}\right) \log \log d} \geq \frac{1}{\zeta(2)} e^{\gamma} .
$$

Proof. The bound (1) is due to Littlewood [207. The bound (2) is due to Chowla [58, Theorem 2].

There is again a factor of 2 difference between the unconditional lower bound and the conditional upper bound. In 1999 Montgomery and Vaughan 222 formulated a model for the general distribution of sizes of Dirichlet character values at $s=1$, and based on it they advanced very precise conjectures in favor of the lower bounds being the correct answer, as follows.

Conjecture 3.9.7 (Montgomery and Vaughan 1999). (1) For each $\epsilon>0$, for all $D \geq D(\epsilon)$ there holds

$$
\max _{d \leq D} L\left(1, \chi_{-d}\right) \leq e^{\gamma} \log \log D+(1+\epsilon) \log \log \log D .
$$

(2) For each $\epsilon>0$, for all $D \geq D(\epsilon)$ there holds

$$
\max _{d \leq D} \frac{1}{L\left(1, \chi_{-d}\right)} \leq \frac{1}{\zeta(2)} e^{\gamma} \log \log D+O\left(\frac{1}{(\log \log D)(\log \log \log D)}\right) .
$$

In 2003 Granville and Soundararajan 142 obtained detailed probabilistic estimates for the number of characters having extreme values $e^{\gamma} \tau$. Their results imply unconditionally that there are infinitely many $d$ with

$$
L\left(1, \chi_{-d}\right) \geq e^{\gamma}(\log \log d+\log \log \log d-\log \log \log \log d-10) .
$$

In their later paper [143, Theorem 3] they sketched a proof that for any fixed $A \geq 10$ for all sufficiently large primes $q$ there are at least $q^{1-\frac{1}{A}}$ Dirichlet characters $\chi(\bmod q)$ such that

$$
|L(1, \chi)| \geq e^{\gamma}(\log \log d+\log \log \log d-\log \log \log \log d-\log A+O(1)) .
$$

Finally we remark on a related problem, concerning the distribution of the phase $\arg (\zeta(1+i t))$. In 1972 Pavlov and Faddeev [243] observed that the phase of $\zeta(1+i t)$ appears in the scattering matrix data for the hyperbolic Laplacian operator acting on the modular surface $X(1)=P S L(2, \mathbb{Z}) \backslash \mathbb{H}$, where $\mathbb{H}=\{z=$ $x+i y: y=\operatorname{Im}(z)>0\}$ denotes the upper half-plane. Then in 1980 Lax and Phillips [199, Section Theorem 7.19] treated this case as an example of their version of scattering theory for automorphic functions (given in [198]). Recently Y. Lamzouri [194] obtained interesting results on the joint distribution of the modulus and phase $(|\zeta(1+i t)|, \arg (\zeta(1+i t)))$. 
3.10. Euler's constant and random permutations: cycle structure. Let $S_{N}$ denote the symmetric group of all permutations on $[1, N]:=\{1,2,3, \ldots, N\}$. By random permutation we mean an element $\sigma \in S_{N}$ drawn with the uniform distribution, picked with probability $\frac{1}{N !}$. We view $\sigma$ as a product of distinct cycles and let $c_{j}(\sigma)$ count the number of cycles of length $j$ in $\sigma$. Three interesting statistics on the cycle structure of a permutation $\sigma \in S_{N}$ are its total number of cycles

$$
n(\sigma):=\sum_{j=1}^{n} c_{j}
$$

the length of its longest cycle

$$
M(\sigma):=\max \left\{j: c_{j}>0\right\},
$$

and the length of its shortest cycle

$$
m(\sigma):=\min \left\{j: c_{j}>0\right\} .
$$

Euler's constant $\gamma$ appears in connection with the the distributions of each of these statistics, viewed as random variables on $S_{N}$.

In 1939 J. Touchard [301, p. 247] expressed the distribution of cycle lengths using exponential generating function methods. In 1944 Goncharov [135, Trans. pp. 3134] (announced 1942 [134]) also gave generating functions, derived in a probabilistic context. He showed that if one lets $c(N, k)$ denote the number of permutations in $S_{N}$ having exactly $k$ cycles, then one has the generating function

$$
\sum_{k=1}^{N} c(N, k) x^{k}=x(x+1)(x+2) \cdots(x+N-1) .
$$

Goncharov computed the mean and variance of the number of cycles $n(\sigma)$, as follows.

Theorem 3.10.1 (Touchard 1939, Goncharov 1944). Draw a random permutation $\sigma$ from the symmetric group $S_{N}$ on $N$ elements with the uniform distribution.

(1) The expected value of the number of cycles in $\sigma$ is

$$
E[n(\sigma)]=H_{N}=\sum_{j=1}^{N} \frac{1}{j} .
$$

In particular one has the estimate

$$
E[n(\sigma)]=\log N+\gamma+O\left(\frac{1}{N}\right) .
$$

(2) The variance of the number of cycles in $\sigma$ is

$$
\operatorname{Var}[n(\sigma)]:=E\left[(n(\sigma)-E[n(\sigma)])^{2}\right]=H_{N}-H_{N, 2}=\sum_{j=1}^{N} \frac{1}{j}-\sum_{j=1}^{N} \frac{1}{j^{2}} .
$$

In particular one has the estimate

$$
\sqrt{\operatorname{Var}[n(\sigma)]}=\sqrt{\log N}+\left(\frac{\gamma}{2}-\frac{\pi^{2}}{12}\right) \frac{1}{\sqrt{\log N}}+O\left((\log N)^{-\frac{3}{2}}\right) .
$$


Proof. In 1939 Touchard [301, p. 291] obtained the formula $E[n(\sigma)]=H_{N}$. In 1944 Goncharov [135, Section 15] derived both (1) and (2), using (3.10.1). A similar proof was given in 1953 by Greenwood [144, p. 404]. Note also that for all $J \geq 1$, the $J$ th moment of $n(\sigma)$ is given by

$$
E\left[n(\sigma)^{J}\right]=\left.\frac{1}{N !}\left(x \frac{d}{d x}\right)^{J} M_{N}(x)\right|_{x=1} .
$$

It follows using (3.10.1) that the $J$ th moment can be expressed as a polynomial in the $m$-harmonic numbers $H_{N, m}$ for $1 \leq m \leq J$.

Goncharov [135, Section 16] used the moments to show furthermore that the normalized random variables $\bar{n}(\sigma):=\frac{n(\sigma)-E[n(\sigma)]}{\sqrt{\operatorname{Var}[n(\sigma)]}}$ satisfy a central limit theorem as $N \rightarrow \infty$, i.e. they converge in distribution to standard unit normal distribution.

Goncharov 135 also obtained information on the distribution of the maximum cycle $M(\sigma)$ as well. Define the scaled random variable $L_{1}^{(N)}:=\frac{1}{N} M(\sigma)$. Then, as $N \rightarrow \infty$, Goncharov showed these random variables have a limiting distribution, with the convergence in distribution $L_{1}^{(N)} \rightarrow_{d} \mathbb{L}_{1}$, in which $\mathbb{L}_{1}$ is the probability distribution supported on $[0,1]$ whose cumulative distribution function

$$
F_{1}(\alpha):=\operatorname{Prob}\left[0 \leq \mathbb{L}_{1} \leq \alpha\right]
$$

is given for $0 \leq \alpha \leq 1$ by

$$
F_{1}(\alpha)=1+\sum_{k=1}^{\left\lfloor\frac{1}{\alpha}\right\rfloor} \frac{(-1)^{k}}{k !} \int_{\substack{t_{1}+\cdots+t_{k} \leq \frac{1}{\alpha} \\ t_{1}, \ldots, t_{k} \geq 1}} \frac{d t_{1}}{t_{1}} \frac{d t_{2}}{t_{2}} \cdots \frac{d t_{n}}{t_{n}},
$$

with $F_{1}(\alpha)=1$ for $\alpha \geq 1$. Much later in 1976 Knuth and Trabb-Pardo [180, Section 10] showed that this distribution is connected to the Dickman function $\rho(u)$ in Section 3.5 by

$$
F_{1}(\alpha)=\rho\left(\frac{1}{\alpha}\right), 0 \leq \alpha \leq 1,
$$

and this yields the previously given formula (3.5.3). From this connection we deduce that this distribution has a continuous density $f_{1}(\alpha) d \alpha$ given by

$$
f_{1}(\alpha)=-\rho^{\prime}\left(\frac{1}{\alpha}\right) \frac{1}{\alpha^{2}}=\rho\left(\frac{1-\alpha}{\alpha}\right) \frac{1}{\alpha},
$$

with the last equality deduced using the differential-difference equation (2) for $\rho(u)$.

In 1966 Shepp and Lloyd 268, obtained detailed information on the distribution of both $m(\sigma)$ and $M(\sigma)$ and also of the $r$ th longest and $r$ th shortest cycles. Euler's constant appears in the distributions of longest and shortest cycles, as follows.

Theorem 3.10.2 (Shepp and Lloyd 1966). Pick a random permutation $\sigma$ on $N$ elements with the uniform distribution.

(1) Let $M_{r}(\sigma)$ denote the length of the rth longest cycle under iteration of the permutation $\sigma \in S_{N}$. Then the kth moment $E\left[M_{r}(\sigma)^{k}\right]$ satisfies, for $k \geq 1$ and $r \geq 1$, as $N \rightarrow \infty$,

$$
\lim _{N \rightarrow \infty} \frac{E\left[M_{r}(\sigma)^{k}\right]}{N^{k}}=G_{r, k}
$$

for positive limiting constants $G_{r, k}$. These constants are explicitly given by

$$
G_{r, k}=\int_{0}^{\infty} \frac{x^{k-1}}{k !} \frac{E i(-x)^{r-1}}{(r-1) !} e^{-E i(-x)} e^{-x} d x,
$$


in which $\operatorname{Ei}(-x)=\int_{x}^{\infty} \frac{e^{-t}}{t} d t$ is the exponential integral. They satisfy, for fixed $k$, as $r \rightarrow \infty$,

in which $\gamma$ is Euler's constant.

$$
\lim _{r \rightarrow \infty}(k+1)^{r} G_{r, k}=\frac{1}{k !} e^{-k \gamma},
$$

(2) Let $m_{r}(\sigma)$ denote the length of the rth shortest cycle of the permutation $\sigma \in S_{N}$. Then the expected value $E\left[m_{r}(\sigma)\right]$ satisfies, as $N \rightarrow \infty$,

$$
\lim _{N \rightarrow \infty} \frac{E\left[m_{r}(\sigma)\right]}{(\log N)^{r}}=\frac{1}{r !} e^{-\gamma}
$$

Furthermore the kth moment $E\left[m_{r}(\sigma)^{k}\right]$ for $k \geq 2$ and $r \geq 1$ satisfies, as $N \rightarrow \infty$,

$$
\lim _{N \rightarrow \infty} \frac{E\left[m_{r}(\sigma)^{k}\right]}{N^{k-1}(\log N)^{r-1}}=\frac{1}{(r-1) !} \int_{0}^{\infty} \frac{x^{k-1}}{(k-1) !} e^{E i(-x)} e^{-x} d x .
$$

Proof. The exponential integral Ei(x), defined for $x<0$ is given in (2.5.13). Here (1) appears in Shepp and Lloyd [268, (13) and (14) ff., p. 347]. Here (2) appears as [268, (22), p. 352].

Shepp and Lloyd 268, also obtained the limiting distribution of the $r$ th longest cycle as $N \rightarrow \infty$. In terms of the scaled variables $L_{r}^{(N)}:=\frac{1}{N} M_{r}(\sigma)$, they deduced the convergence in distribution

$$
L_{r}^{(N)} \longrightarrow_{d} \mathbb{L}_{r},
$$

in which $\mathbb{L}_{r}$ is a distribution supported on $\left[0, \frac{1}{r}\right]$ whose cumulative distribution function

is given by

$$
F_{r}(\alpha):=\operatorname{Prob}\left[0 \leq \mathbb{L}_{1} \leq \alpha\right], \quad 0 \leq \alpha \leq \frac{1}{r}
$$

$$
F_{r}(\alpha)=1+\sum_{k=r}^{\left\lfloor\frac{1}{\alpha}\right\rfloor} \frac{(-1)^{k-r+1}}{(r-1) !(k-r) ! k} \int_{\substack{t_{1}+\cdots+t_{k} \leq \frac{1}{\alpha} \\ t_{1}, \ldots, t_{k} \geq 1}} \frac{d t_{1}}{t_{1}} \frac{d t_{2}}{t_{2}} \cdots \frac{d t_{n}}{t_{n}},
$$

and we set $F_{r}(\alpha)=1$ for $\frac{1}{r} \leq \alpha<\infty$. This extends to general $r$ the case $r=1$ treated by Goncharov [135]. In 1976 Knuth and Trabb-Pardo [180, Section 4] defined $\rho_{r}(u)$ by $\rho_{r}(u):=F_{r}\left(\frac{1}{u}\right)$, so that $\rho_{1}(u)=\rho(u)$ by (3.10.3). The functions $\rho_{r}(u)$ for $r \geq 1$ are uniquely determined by the following conditions.

(1) Initial condition. For $0 \leq u \leq 1$, it satisfies

$$
\rho_{r}(u)=1 \text {. }
$$

(2) Differential-difference equation. For $u>1$,

$$
u \rho_{r}^{\prime}(u)=-\rho_{r}(u-1)+\rho_{r-1}(u-1),
$$

with the convention $\rho_{0}(u) \equiv 0$.

Knuth and Trabb-Pardo formulated these conditions in the integral equation form

$$
\rho_{r}(u)=1-\int_{1}^{u}\left(\rho_{r}(t-1)-\rho_{r-1}(t-1)\right) \frac{d t}{t} .
$$

One may directly check that these equations imply $\rho_{r}(u)=1$ for $0 \leq u \leq r$.

Associated to the longest cycle distribution is another interesting constant

$$
\lambda:=G_{1,1}=\lim _{N \rightarrow \infty} \frac{E[M(\sigma)]}{N} .
$$


The formula (3.10.4) gives

$$
\lambda=\int_{0}^{\infty} e^{-x-E i(-x)} d x=0.6243299885 \cdots .
$$

This constant $\lambda$ is now named the Golomb-Dickman constant in Finch [116, Section 5.4], for the following reasons. It was first encountered in 1959 work of Golomb, Welch, and Goldstein [129] in a study of the asymptotics of statistics associated to shift register sequences. Their paper expressed this constant by an integral involving a solution to a differential-difference equation, described later in Golomb [131, p. 91, (33)]. In 1964 Golomb [130] asked for a closed form for this constant, and Shepp and Lloyd's work answered it with (3.10.7) above. It was evaluated to 53 places by Mitchell [221] in 1968. In 1976 Knuth and Trabb-Pardo [180, Section 10] showed that

$$
\lambda=1-\int_{1}^{\infty} \frac{\rho(u)}{u^{2}} d u,
$$

where $\rho(x)$ is the Dickman function discussed in Section 3.5. This associates the name of Dickman with this constant. In fact the constant already appears in de Bruijn's 1951 work [51, (5.2)], where he showed

$$
\lambda=\int_{0}^{\infty} \frac{\rho(u)}{(u+1)^{2}} d u,
$$

as noted Wheeler [316, p. 516]. According to Golomb [131, p. 192] another integral formula for this constant, found by Nathan Fine, is

$$
\lambda=\int_{0}^{1} e^{L i(x)} d x
$$

in which $\operatorname{Li}(x):=\int_{0}^{x} \frac{d t}{\log t}$ for $0 \leq x<1$. In 1990 Wheeler [316, pp. 516-517] established the formula

$$
\lambda=\int_{0}^{\infty} \frac{\rho(u)}{u+2} d u
$$

noted empirically earlier by Knuth and Trabb-Pardo, and also the formula

$$
\lambda=e^{\gamma} \int_{0}^{\infty} e^{-\operatorname{Ein}(t)-2 t} d t
$$

in which $\operatorname{Ein}(t)$ is given in 3.5 .5 . The Golomb-Dickman constant $\lambda$ is not known to be related to either Euler's constant $\gamma$ or the Euler-Gompertz constant $\delta$, and it is not known whether it is irrational or transcendental.

The Golomb-Dickman constant $\lambda$ appears as a basic statistic in the limit distribution $\mathbb{L}_{1}$ for the scaled longest cycle, whose expected value is given by

$$
E\left[\mathbb{L}_{1}\right]=\int_{0}^{1} \alpha d F_{1}(\alpha)=-\int_{0}^{1} \frac{1}{\alpha} \rho^{\prime}\left(\frac{1}{\alpha}\right) d \alpha .
$$

Letting $x=\frac{1}{\alpha}$, we obtain

$$
E\left[\mathbb{L}_{1}\right]=-\int_{1}^{\infty} \frac{\rho^{\prime}(x)}{x} d x=\int_{1}^{\infty} \rho(x-1) \frac{d x}{x^{2}}=\lambda ;
$$

see [180, (9.2)].

In 1996 Gourdon [138, Chapter VII, Théorème 2] determined a complete asymptotic expansion of the expected values $E[M(\sigma)]$ as $N \rightarrow \infty$ in powers of $\frac{1}{N}$, in 
which both the Golomb-Dickman constant and Euler's constant appear, with the initial terms being

$$
E[M(\sigma)]=\lambda N+\frac{1}{2} \lambda-\frac{e^{\gamma}}{24} \frac{1}{N}+O\left(\frac{1}{N^{2}}\right) .
$$

The omitted higher order terms for $k \geq 2$ in Gourdon's asymptotic expansion are of the form $\frac{P_{k}(N)}{N^{k}}$, in which each $P_{k}(N)$ is an oscillatory function of $N$ which is periodic with an integer period $p_{k}$ that grows with $k$ as $k \rightarrow \infty$.

A result in a different direction relates Euler's constant to the probability of all cycles having distinct lengths as $N \rightarrow \infty$. This result follows from work of $\mathrm{D}$. $\mathrm{H}$. Lehmer 200].

Theorem 3.10.3 (Lehmer 1972). Draw a random permutation $\sigma$ on $N$ elements with the uniform distribution. Let $P_{d}(N)$ denotes the probability that the cycles of $\sigma$ have distinct lengths, then

$$
\lim _{N \rightarrow \infty} P_{d}(N)=e^{-\gamma} .
$$

Proof. The cycle lengths of a permutation $\sigma$ form a partition $\lambda=\left(1^{c_{1}}, 2^{c_{2}}, \ldots, N^{c_{N}}\right)$ of $N$ ( written $\lambda \vdash N$ ), having $n(\lambda)=c_{1}+c_{2}+\cdots+c_{N}$ cycles with cycle length $j$ occurring $c_{j}$ times. The number of permutations $N(\lambda)$ giving rise to a given partition $\lambda$ is

$$
N(\lambda)=\frac{N !}{c_{1} ! c_{2} ! \cdots c_{N} ! 1^{c_{1}} 2^{c_{2}} \cdots N^{c_{N}}},
$$

and by definition we have

$$
\frac{1}{N !} \sum_{\lambda \vdash N} N(\lambda)=1
$$

In the case that all cycles are of distinct lengths, then all $c_{i}=0$ or 1 , and the formula for $N(\lambda)$ becomes

$$
N(\lambda)=\frac{N !}{a_{1} a_{2} \cdots a_{n}},
$$

in which $a_{1}<a_{2}<\cdots<a_{n}$ with $n=n(\lambda)$ are the lengths of the cycles. (The division by $a_{i}$ reflects the fact that a cyclic shift of a cycle is the same cycle.) Lehmer 200] assigned weights $\left(1^{c_{1}} 2^{c_{2}} \cdots N^{c_{N}}\right)^{-1}$ to each partition, and counted different sets of such weighted partitions. Theorem 2 counted partitions with distinct summands, in his notation

$$
W_{N}^{*}:=\sum_{\lambda \vdash N}^{\prime} \frac{1}{a_{1} a_{2} \cdots a_{n}},
$$

with the prime indicating the sum runs over partitions having distinct parts. and showed that $W_{N}^{*} \rightarrow e^{-\gamma}$ as $N \rightarrow \infty$. (Lehmer's weights agree with the weights assigned to partitions from the uniform distribution on $S_{N}$ only for those partitions having distinct parts.)

3.11. Euler's constant and random permutations: shortest cycle. There are striking parallels between the distribution of cycles of random permutations of $S_{N}$ and the distributions of factorizations of random numbers in an interval $[1, n]$, particularly concerning the number of these having factorizations of restricted types, allowing either factorizations with only small factors (treated in Section 3.5) or having only large prime factors (treated in Section 3.6) where we take $N$ to be on the order of $\log n$. This relation was first observed by Knuth and Trabb-Pardo 
[180, whose 1976 work (already mentioned in Section 3.5] and Section 3.10) was done to analyze the performance of an algorithm for factoring integers by trial division. They observed in [180, Section 10, p. 344]:

Therefore, if we are factoring the digits of a random $m$-digit number, the distribution of the number of digits in its prime factors is approximately the same as the distribution of the cycle lengths in a random permutation on $m$ elements! (Note that there are approximately $\ln m$ factors, and $\ln m$ cycles.)

They uncovered this connection after noticing that the Shepp-Lloyd formula for Golomb's constant $\lambda$ in (3.10.7), as evaluated by Mitchell [221], agreed to ten places with their calculation of Dickman's constant, as defined by (3.10.8). A comparison of the $k$ th longest cycle formulas of Shepp and Lloyd in Theorem 3.10.2 for cycles of length $\alpha N$ then revealed matching relatives $\rho_{k}(x)$ of the Dickman function (defined in Section 3.5). These parallels extend to the appearance of the Buchstab function in the distribution of random permutations having no short cycle, as we describe below.

Concerning the shortest cycle, recall first that a special case of Theorem 3.10 .2 (2) shows that the expected length of the shortest cycle is

$$
E[m(\sigma)]=e^{-\gamma} \log N(1+o(1)) \text { as } N \rightarrow \infty,
$$

It is possible to get exact combinatorial formulas for the probability

$$
P(N, m):=\operatorname{Prob}\left[m(\sigma) \geq m: \sigma \in S_{N}\right]
$$

that a permutation has no cycle of length shorter than $m$. There are several limiting behaviors of this probability as $N \rightarrow \infty$, depending on the way $m=m(N)$ grows as $N \rightarrow \infty$. There are three regimes, first, where $m$ is constant, second where $m(N)=\alpha N$ grows proportionally to $N$, with $0<\alpha \leq 1$ (i.e. only long cycles occur) and third, the intermediate regime, where $m(N) \rightarrow \infty$ but $\frac{m(N)}{N} \rightarrow 0$ as $N \rightarrow \infty$.

First, suppose that $m(N)=m$ is constant. This case has a long history. The derangement problem (problème des ménages), concerns the probability that a permutation has no fixed point, which is the case $m=2$. It was raised by Rémond de Montmort [256] in 1708 and solved by Nicholas Bernoulli in 1713; see [257, pp. 301303]. The derangement problem was also solved by Euler in 1753 [102, E201], who was unaware of the earlier work. The well known answer is that

$$
P(N, 2)=\sum_{j=0}^{N} \frac{(-1)^{j}}{j !},
$$

and this exact formula yields the result

$$
\lim _{N \rightarrow \infty} P(N, 2)=\frac{1}{e} .
$$

In 1952 Gruder [147] gave a generalization for permutations having no cycle shorter than a fixed $m$, as follows.

Theorem 3.11.1 (Gruder 1952). For fixed $m \geq 1$ there holds

$$
\lim _{N \rightarrow \infty} \operatorname{Prob}\left[m(\sigma) \geq m: \sigma \in S_{N}\right]=e^{-H_{m-1}},
$$

in which $H_{m}$ denotes the mth harmonic number. 
Proof. Let $P_{N}(m, k)$ count the number of permutations of $N$ having exactly $k$ cycles, each of length at least $m$, and set $P_{N}(m)=\sum_{k=1}^{N} P_{N}(m, k)$, so that $P(N, m)=\frac{P_{N}(m)}{N !}$. These are given in the exponential generating function

$$
\begin{aligned}
\sum_{N=0}^{\infty} \frac{z^{N}}{N !}\left(\sum_{k=1}^{N} P_{N}(m, k) u^{k}\right) & =\exp \left[u\left(\log \frac{1}{1-x}-\sum_{j=1}^{m-1} \frac{x^{j}}{j}\right)\right] \\
& =\frac{\exp \left[-u\left(x+\frac{x^{2}}{2}+\cdots+\frac{x^{m-1}}{m-1}\right)\right]}{(1-x)^{u}} .
\end{aligned}
$$

Gruder [147, Section 8, (85)] derives from the case $u=1$,

$$
\sum_{N=0}^{\infty} P_{N}(m) \frac{x^{N}}{N !}=\frac{\exp \left(-x-\frac{x^{2}}{2}-\cdots-\frac{x^{m-1}}{m-1}\right)}{1-x} .
$$

Using this fact, Gruder [147, Section 9, (94)] derives $\lim _{N \rightarrow \infty} \frac{N !}{P_{N}(m)}=e^{H_{m-1}}$, giving the result.

Gruder also observes that one obtains Euler's constant from these values via the scaling limit

$$
\lim _{m \rightarrow \infty} \lim _{N \rightarrow \infty} m P(N, m)=e^{-\gamma} .
$$

An asymptotic expansion concerning convergence to the limit 3.11.1) for constant $m$ is now available in general circumstances. Panario and Richmond [242, Theorem $3,(9)$ ], give such an asymptotic expansion for the $r$ th largest cycle being $\geq m$ for a large class of probability models.

Second, for the intermediate range where $m=m(N) \rightarrow \infty$ with $\frac{m}{N} \rightarrow 0$ as $N \rightarrow \infty$, there is the following striking universal limit estimate, in which $e^{-\gamma}$ appears.

Theorem 3.11.2 (Panario and Richmond 2001). Consider permutations on $N$ letters whose shortest cycle $m(\sigma) \geq m$. Suppose that $m=m(N)$ depends on $N$ in such a way that $m(N) \rightarrow \infty$ and $\frac{m(N)}{N} \rightarrow 0$ both hold as $N \rightarrow \infty$. Under these conditions we have

$$
\operatorname{Prob}\left[m(\sigma) \geq m(N): \sigma \in S_{N}\right] \sim \frac{e^{-\gamma}}{m}, \quad \text { as } N \rightarrow \infty .
$$

Proof. This result is deducible from a general asymptotic result of Panario and Richmond [242, Theorem 3, (11)], which applies to distribution of the $r$ th largest cycle of a logarithmic combinatorial structure.

Third, we consider the large range, concerning those permutations having shortest cycle of length at least a constant fraction $\alpha N$ of the size of the permutation. Here the resulting scaled density involves the Buchstab function $\omega(u)$ treated in Section 3.6. with $\alpha=\frac{1}{u}$, with $u>1$.

Theorem 3.11.3 (Panario and Richmond 2001). Consider permutations on $N$ letters whose shortest cycle $m(\sigma) \geq m$. Suppose that $m=m(N)$ depends on $N$ in such a way that $\frac{m(N)}{N} \rightarrow \alpha$ holds as $N \rightarrow \infty$, with $0<\alpha \leq 1$. Under these conditions we have, for fixed $0<\alpha \leq 1$, that

$$
\operatorname{Prob}\left[m(\sigma)>\alpha N: \sigma \in S_{N}\right] \sim \omega(1 / \alpha) \frac{1}{\alpha N}, \quad \text { as } N \rightarrow \infty,
$$

where $\omega(u)$ denotes the Buchstab function. 
Proof. This result follows from a general result of Panario and Richmond [242, Theorem 3, (10)], which gives an asymptotic expansion for the $r$ th largest cycle. The proof for this case uses in part methods of Flajolet and Odlyzko [120].

The matching of the large range estimate (3.11.2) as $\alpha \rightarrow 0$ with the intermediate range estimate follows from the limiting behavior of the Buchstab function $\omega(u) \rightarrow e^{-\gamma}$ as $u \rightarrow \infty$. One may compare Theorem 3.11 .2 with the sieving result in Theorem 3.6.1. They are quite parallel, and in both cases the factor $e^{-\gamma}$ arises from the limiting asymptotic behavior of the Buchstab function.

The appearance of the Buchstab function in the sieving models in Section 3.6 and the permutation cycle structure above is precisely accounted for in stochastic models developed by Arratia, Barbour, and Tavaré 17, 18 in the late 1990s, which they termed Poisson-Dirichlet processes (and which we do not define here). They showed ([17, [19, Sections 1.1, 1.2]) that (logarithmically scaled) factorizations of random integers, and (scaled) cycle structures of random permutations as $N \rightarrow \infty$ lead to identical limiting processes. To describe the scalings, one considers an ordered prime factorization of an random integer $m=\prod_{i=1}^{n} p_{i}$, with $2 \leq p_{1} \leq p_{2} \leq \cdots \leq p_{k}$. Let $k:=\Omega(m)$ count the number of prime factors of $m$ with multiplicity, one defines the logarithmically scaled quantities

$$
\bar{a}_{i}^{*}:=\frac{\log p_{i}}{\log m}, 1 \leq i \leq k .
$$

The random quantities drawn are $\left(\bar{a}_{1}^{*}, \bar{a}_{2}^{*}, \ldots, \bar{a}_{k}^{*}\right)$, where $k$ itself is also a random variable, and these quantities sum to 1 . To a random permutation $\sigma$ of $S_{N}$ let $a_{1} \leq a_{2} \leq \cdots \leq a_{n(\sigma)}$ represent the lengths of the cycles arranged in increasing order. Then associate to $\sigma$ the normalized cycle lengths

$$
\overline{a_{i}}:=\frac{a_{i}}{N}, 1 \leq i \leq n \text {. }
$$

Here $n=n(\sigma)$ is a random variable, and these quantities also sum to 1. Arratia, Barbour, and Tavaré [18] showed that in both cases, as $N \rightarrow \infty$ (resp. $n \rightarrow \infty$ ) these random variables converge to a limiting stochastic process, a Poisson-Dirichlet process of parameter 1 . They furthermore noted ([19, p. 34]) that both models required exactly the same normalizing constant: $e^{-\gamma}$. The existence of a normalizing constant for Poisson-Dirichlet processes was noted in 1977 by Vershik and Shmidt [311, who conjectured it to be $e^{-\gamma}$, and this was proved in 1982 by Ignatov [170].

The model for random cycles applies as well to random factorization of polynomials of degree $N$ over the finite field $G F(q)$, as $q \rightarrow \infty$; see Arratia, Barbour, and Tavaré [16].

3.12. Euler's constant and random finite functions. Euler's constant also appears in connection with the distribution of cycles in a random finite function $F:[1, N] \rightarrow[1, N]$. Iteration of a finite function on a given initial seed leads to iterates of an element having a preperiodic part, followed by arrival at a cycle of the function. Thus not every element of $N$ belongs to a cycle.

In 1968 Purdom and Williams 251] established results for random finite functions that are analogous to results for random permutations given in Section 3.10. They studied the length of the longest and shortest cycles for a random function; note however that the expected length of the longest cycle grows proportionally to $\sqrt{N}$, rather than proportional to $N$ as in the case of a random permutation. 
Theorem 3.12.1 (Purdom and Williams 1968). Pick a random function $F$ : $[1, N] \rightarrow[1, N]$ with the uniform distribution over all $N^{N}$ such functions.

(1) The length $M(F)$ of the longest cycle of $F$ under iteration satisfies

$$
\lim _{N \rightarrow \infty} \frac{E[M(F)]}{\sqrt{N}}=\lambda \sqrt{\frac{\pi}{2}},
$$

in which $\lambda$ is the Golomb-Dickman constant.

(2) Let $m(F)$ denote the length of the shortest cycle under iteration of the function $F$. Then the expected value $E[m(F)]$ satisfies

$$
\lim _{N \rightarrow \infty} \frac{E[m(F)]}{\log N}=\frac{1}{2} e^{-\gamma} .
$$

Proof. For fixed $N$, Purdom and Williams [251 use the notation $E[M(F)]=$ $E_{F_{N}, 1}(\ell):=E[M(F)]$ and $E_{F_{N}, 1}(s):=E[m(F)]$. The result (1) follows from [251, p. 550, second equation from bottom]. Purdom and Williams state a result related to (2) without detailed proof. Namely, the top equation on [251, p. 551] states

where

$$
E[m(F)]=S_{1,1} Q_{n}(1,1)+o\left(Q_{n}(1,1)\right)
$$

Here the values $S_{r, k}$ are taken from Shepp and Lloyd [268], where for $k=1$ they are given by the right-hand side of (3.10.5), and for $k \geq 2$ by the right-hand side of (3.10.6), and in particular $S_{1,1}=e^{-\gamma}$. To estimate $Q_{n}(1,1)$ given by (3.12.1) we use the identity $([251$, p. 550])

$$
Q_{n}(0)=\sum_{j=1}^{n} \frac{(n-1) ! j}{(n-j) ! n^{j}}=1 .
$$

We observe that $Q_{n}(1,1)$ is the expected value of the function $\log j$ with respect to the probability distribution given by the individual terms in $Q_{n}(0)$. One may deduce that $Q_{n}(1,1)=\frac{1}{2} \log n+o(\log n)$ by showing that this probability distribution is concentrated on values $j=\sqrt{n}+o(\sqrt{n})$. This gives (2).

The study of random finite functions is relevant for understanding the behavior of the Pollard [247] "rho" method of factoring large integers. The paper of Knuth and Trabb-Pardo [180] analyzed the performance of this algorithm, but their model used random permutations rather than random functions. An extensive study of limit theorems for statistics for random finite functions was made by V. F. Kolchin [182. A very nice asymptotic analysis of the major statistics for random finite functions is given in Flajolet and Odlyzko [119, Theorems 2 and 3].

3.13. Euler's constant as a Lyapunov exponent. Certain properties of Euler's constant have a formal resemblance to properties of a dynamical entropy. More precisely, the quantity $C=e^{\gamma}$ might be interpretable as the growth rate of some dynamical quantity, with $\gamma=\log C$ being the associated (topological or metric) entropy. Here we present results about the growth rates of the size of products of random matrices having normally distributed entires. These growth rates naturally involve $\gamma$, together with other known constants. 
Before stating results precisely, we remark that products of random matrices often have well defined growth rates, which can be stated either in terms of growth rates of matrix norms or in terms of Lyapunov exponents. Let $\{A(j): j \geq 1\}$ be a fixed sequence of $d \times d$ complex matrices, and let $M(n)=A(n) A(n-1) \cdots A(2) A(1)$ be product of $d \times d$ complex matrices. We define for an initial vector $\mathbf{v} \in \mathbb{C}^{d}$ and a vector norm $\|\cdot\|$, the logarithmic growth exponent of $\mathbf{v}$ to be

$$
\lambda(\mathbf{v}):=\limsup _{n \rightarrow \infty} \frac{1}{n} \log \frac{\|M(n) \mathbf{v}\|}{\|\mathbf{v}\|} .
$$

This value depends on $\mathbf{v}$ and on the particular sequence $\{A(j): j \geq 1\}$ but is independent of the choice of the vector norm. For a fixed infinite sequence $\{A(j): j \geq 1\}$ as $\mathbf{v}$ varies there will at most $d$ distinct values $\lambda_{1}^{*} \leq \lambda_{2}^{*} \leq \cdots \leq \lambda_{d}^{*}$ for this limit.

Now let the sequence $\{A(j): j \geq 1\}$ vary, with each $A(j)$ being drawn independently from a given probability distribution on the set of $d \times d$ matrices. In 1969 Furstenberg and Kesten [122] showed that for almost all vectors $\mathbf{v}$ (off a set of positive codimension) the limiting value $\lambda_{d}^{*}$ takes with probability one a constant value $\lambda_{d}$, provided the distribution satisfied the condition

$$
\int_{X} \log ^{+}\|A(x)\| d \mu(x)<\infty,
$$

where $(X, \Sigma, \mu)$ is the probability space, $\|\cdot\|$ is a fixed matrix norm, required to be submultiplicative, i.e. $\left\|M_{1} M_{2}\right\| \leq\left\|M_{1}|||| M_{2}\right\|$, and where $\log ^{+}|x|:=$ $\max (0, \log |x|)$. A corollary of the multiplicative ergodic theorem proved by Oseledec in 1968 (see [241, [252]) asserts that with probability one all these logarithmic exponents take constant values, provided the probability distribution on random matrices satisfies (3.13.2). These constant values $\lambda_{1} \leq \lambda_{2} \leq \cdots \leq \lambda_{d}$ are called the Lyapunov exponents of the random matrix product, with $\lambda_{d}$ being the maximal Lyapunov exponent. For almost all random products and almost all starting vectors $\mathbf{v}_{0}$, one will have $\lambda(\mathbf{v})=\lambda_{d}$; compare Pollicott [248]. The value $C_{d}:=\exp \left(\lambda_{d}\right)$ may be thought of as approximating the exponential growth rate of matrix norms $\left\|M(n)^{T} M(n)\right\|^{1 / 2}$, as $n \rightarrow \infty$.

In general the Lyapunov exponents are hard to determine. In 1984 J. Cohen and C. M. Newman [63, Theorem 2.5] obtained the following explicit result for normally distributed variables. Note that condition (3.13.2) holds for such variables.

Theorem 3.13.1 (Cohen and Newman 1984). Let $\left\{A(1)_{i j}: 1 \leq i, j \leq d\right\}$ be independent, identically distributed (i.i.d.) normal random variables with mean 0 and variance $s$, i.e. $A(1)_{i j} \sim N\left(0, s^{2}\right)$. Let $\mathbf{v}(0)$ be a nonzero vector in $\mathbb{R}^{d}$. Define for independent random draws

$$
\mathbf{v}(n)=A(n) A(n-1) \cdots A(1) \mathbf{v}(0), n=1,2,3, \ldots,
$$

and consider 31

$$
\lambda(\mathbf{v}(0)):=\lim _{n \rightarrow \infty} \frac{1}{n} \log \|\mathbf{v}(n)\|
$$

\footnotetext{
${ }^{31}$ Cohen and Newman write the left sides of 3.13.3 and 3.13.4 as $\log \lambda$, but here we replace these by the rescaled variables $\lambda\left(\mathbf{v}_{0}\right)$ and $\lambda$, in order to match the definition of Lyapunov exponent given in (3.13.1.
} 
using the Euclidean norm $\|\cdot\|$ on $\mathbb{R}^{d}$. Then with probability one the limit exists in (3.13.3), is nonrandom, is independent of $\mathbf{v}(0)$, and is given by

$$
\lambda=\frac{1}{2}\left[\log \left(s^{2}\right)+\log 2+\psi\left(\frac{d}{2}\right)\right]
$$

in which $\psi(x)$ is the digamma function. Moreover as $n \rightarrow \infty$ the random variables $\frac{1}{\sqrt{n}} \log \left(e^{-\lambda n}\|\mathbf{v}(n)\|\right)$ converge in distribution to $N\left(0, \sigma^{2}\right)$ with

$$
\sigma^{2}=\frac{1}{4} \psi^{\prime}\left(\frac{d}{2}\right)
$$

It is easy to deduce from Cohen and Newman's result the following consequence concerning $d \times d$ (noncommutative) random matrix products.

Theorem 3.13.2. Let $A(j)$ be $d \times d$ matrices with entries drawn as independent identically distributed (i.i.d.) normal random variables with variance 1, i.e. $N(0,1)$. Consider the random matrix product

$$
S(n)=A(n) A(n-1) \cdots A(1),
$$

and let $\|S\|_{F}=\sqrt{\sum_{i, j}\left|S_{i, j}\right|^{2}}$ be the Frobenius matrix norm. Then, with probability one, the growth rate is given by

$$
\lim _{n \rightarrow \infty}\left(\|S(n)\|_{F}^{2}\right)^{\frac{1}{n}}= \begin{cases}2 e^{-\gamma+H_{d / 2-1}} & \text { if } d \text { is even } \\ \frac{1}{2} e^{-\gamma+2 H_{d-1}-H_{(d-1) / 2}} & \text { if } d \text { is odd } .\end{cases}
$$

Here $H_{n}$ denotes the $n$th harmonic number, using the convention that $H_{0}=0$.

Proof. We use an exponentiated version of the formula (3.13.4) in Theorem 3.13.1, setting the variance $s=1$ and multiplying both sides of (3.13.4) by 2 before exponentiating. We obtain, for a nonzero vector $\mathbf{v}(0)$, that with probability 1 ,

$$
\lim _{n \rightarrow \infty}\left(\|S(n) \mathbf{v}(0)\|^{2}\right)^{\frac{1}{n}}=2 e^{\psi\left(\frac{d}{2}\right)},
$$

where $\|\cdot\|$ is the Euclidean norm on vectors. To obtain the Frobenius norm bound, we use the identity $\|S\|_{F}^{2}=\sum_{j=1}^{d}\left\|S \mathbf{e}_{j}\right\|^{2}$ where $\mathbf{e}_{i}$ is the standard orthonormal basis of column vectors. On taking vectors $\mathbf{v}_{j}(0)=\mathbf{e}_{j}$, we obtain

$$
\|S(n)\|_{F}^{2}=\sum_{j=1}^{d}\left\|S(n) \mathbf{v}_{j}(0)\right\|^{2}
$$

and using (3.13.6) and the fact that $\lim _{n \rightarrow \infty} d^{\frac{1}{n}}=1$, we obtain with probability 1 that

$$
\lim _{n \rightarrow \infty}\left(\|S(n)\|_{F}^{2}\right)^{\frac{1}{n}}=2 e^{\psi\left(\frac{d}{2}\right)} .
$$

On applying the digamma formulas for $\psi\left(\frac{d}{2}\right)$ given in Theorem 3.1.1, with the convention $H_{0}=0$, and noting for $d=2 m+1$ that

$$
2 e^{\psi(d / 2)}=\frac{1}{2} e^{-\gamma+2 H_{2 m-1}-H_{m-1}}=\frac{1}{2} e^{-\gamma+2 H_{2 m}-\frac{2}{2 m}-H_{m-1}}=\frac{1}{2} e^{-\gamma+2 H_{2 m}-H_{m}},
$$

we obtain (3.13.5). 
Rephrased in terms of Lyapunov exponents, Theorem 3.13.2 says that the corresponding $d \times d$ matrix system, for odd dimension $d$ has top Lyapunov exponent

$$
\lambda_{d}=\frac{1}{2}\left(-\gamma-\log 2+2 H_{d-1}-H_{\frac{d-1}{2}}\right),
$$

and for even dimension $d$ has top Lyapunov exponent

$$
\lambda_{d}=\frac{1}{2}\left(-\gamma+\log 2+H_{\frac{d}{2}-1}\right) .
$$

The simplest expressions occur in dimension $d=1$, giving $\lambda_{1}=\frac{1}{2}(-\gamma-\log 2)$, and in $d=2$, giving $\lambda_{2}=\frac{1}{2}(-\gamma+\log 2)$.

Note that any real number can be obtained as a maximal Lyapunov exponent in Theorem 3.13 .1 simply by adjusting the variance parameter $s$ properly in the normal distribution $N(0, s)$. It follows that the content of the specific formulas in Theorem 3.13 .2 is in part arithmetic, where one specifies the variance $s$ of the normal distributions to be a fixed rational number. Moreover, if we rescale $s$ to choose normal distributions $N(0, s)$ with variance $s=2$ in dimension 1 and with variance $s=\frac{1}{2}$ in dimension 2, then we obtain the Lyapunov exponents $\lambda_{d}=\frac{1}{2}(-\gamma)$ in these dimensions.

Returning to the theme of Euler's constant being (possibly) interpretable as a dynamical entropy, one approach to the Riemann hypothesis suggests that it may be related to an (as yet unknown) arithmetical dynamical system; cf. Deninger 67], [68, 69], 70]. There is a statistical mechanics interpretation of the Riemann zeta function as a partition function given in Bost and Connes [40, with subsequent developments described in Connes and Marcolli 64. For some other views on arithmetical dynamical systems related to zeta functions, see Lagarias 190, 192 and Lapidus [197, Chapter 5]. Here we note the coincidence that in formulations of the Riemann hypothesis given earlier in Theorems 3.7.1 and 3.7.3, a "growth rate" $e^{ \pm \gamma}$ naturally appears.

3.14. Euler's constant and periods. In 2001 M. Kontsevich and D. Zagier [186] defined a period to be a complex number whose real and imaginary parts are values of absolutely convergent integrals of rational functions with rational coefficients, over domains in $\mathbb{R}^{n}$ given by polynomial inequalities with rational coefficients. More conceptually these are values of integrals of an algebraic differential form with algebraic coefficients, integrated over an algebraic cycle; see Kontsevich [185, Section 4.3]. We will call such an integral a period integral. They observed that for the real part one can reduce such integrals to the case where all coefficients are rational numbers, all differentials are top degree, and integrals are taken over divisors with normal crossings defined over $\mathbb{Q}$. In particular they introduced a ring $\mathcal{P}$ of effective periods, which includes the field of all algebraic numbers $\overline{\mathbb{Q}}$ as a subring, as well as a ring of extended periods $\hat{\mathcal{P}}$ obtained by adjoining to $\mathcal{P}$ the constant $\frac{1}{2 \pi i}$, which conjecturally is not a period.

One has, for $n \geq 2$ the representation

$$
\zeta(n)=\int_{0<t_{1}<t_{2}<\cdots<t_{n}<1} \frac{d t_{1}}{1-t_{1}} \frac{d t_{2}}{t_{2}} \cdots \frac{d t_{n}}{t_{n}}
$$


as a period integral. Thus convergent positive integer zeta values are periods. More generally, one can represent all (convergent) multiple zeta values

$$
\zeta\left(n_{1}, n_{2}, \ldots, n_{k}\right):=\sum_{m_{1}>m_{2}>\cdots>m_{k}>0} \frac{1}{m_{1}^{n_{1}} m_{2}^{n_{2}} \cdots m_{k}^{n_{k}}}
$$

at positive integer arguments as period integrals. Other period integrals are associated with special values of $L$-functions, a direction formulated by Deligne [65. An overview paper of Zagier 320 gives a tour of many places that multiple zeta values appear.

Some other examples of periods given by Kontsevich and Zagier are the values $\log \alpha$ for $\alpha$ a positive real algebraic number. In addition the values $\left(\Gamma\left(\frac{p}{q}\right)\right)^{q}$ where $\frac{p}{q}$ is a positive rational number are periods; see Kontsevich and Zagier [186, p. 775] and André [9, Chapter 24]. This can be deduced using Euler's Beta integral (2.3.1). Another well known example is $([20,(5.11)])$

$$
\int_{0}^{1} \frac{d t}{\sqrt{1-t^{4}}}=\frac{\left(\Gamma\left(\frac{1}{4}\right)\right)^{2}}{\sqrt{32 \pi}}
$$

giving a period of an elliptic curve with complex multiplication.

Periods arise in many places. They arise in evaluating Feynman integrals in quantum field theory calculations. For more work on periods and zeta values in this context, see Belkale and Brosnan [28. Periods appear as multiple zeta values in Drinfeld's associator; cf. Drinfeld [76, [77, [78. Periods appear in Vassiliev's knot invariants, due to the connection found by Kontsevich [184]; see Bar-Natan [25]. They appear in expansions of Selberg integrals; cf. Terasoma 296. They appear in the theory of motives as periods of mixed Tate motives; cf. Terasoma [297, [298] and Deligne and Goncharov [66]. Other constants that are periods are values of polylogarithms at integers. Algebraic multiples of beta values $B\left(\frac{r}{n}, \frac{s}{n}\right)$ occur as periods of differentials on abelian varieties; cf. Gross [146, Rohrlich appendix]. In 2002 P. Cartier [55] gave a Bourbaki seminar exposé surveying multiple zeta values, polylogarithms, and related quantities.

There is a good deal known about the irrationality or transcendence of specific periods. The transcendence of $\zeta(2 n)$ for $n \geq 1$ is immediate from their expression as powers of $\pi$. For odd zeta values, in 1978 Apéry ([10, [11]) established that $\zeta(3)$ is irrational; see van der Poorten [249] and Section 3.15] for more detail. In 1979 F. Beukers 31] gave an elegant proof of the irrationality of $\zeta(3)$ suggested by the form of Apéry's proof, showing that certain integer linear combinations of period integrals defined for integer $r, s \geq 0$ by

$$
I_{r, s}:=\int_{0}^{1} \int_{0}^{1} \frac{-\log x y}{1-x y} x^{r} y^{s} d x d y=\int_{0}^{1} \int_{0}^{1} \int_{0}^{1} \frac{x^{r} y^{s}}{1-(1-x y) z} d z d x d y
$$

were very small. He evaluated

$$
I_{0,0}=\int_{0}^{1} \int_{0}^{1} \frac{-\log x y}{1-x y} d x d y=2 \zeta(3)
$$

and for $r \geq 1$,

$$
I_{r, r}=2\left(\zeta(3)-\frac{1}{1^{3}}-\frac{1}{2^{3}}-\cdots-\frac{1}{r^{3}}\right)=2\left(\zeta(3)-H_{n, 3}\right) .
$$

As a necessary part of the analysis he also showed for $r>s$ that each $I_{r, s}=I_{s, r}$ is a rational number with denominator dividing $D_{r}^{3}$ where $D_{r}$ is the least common 
multiple $[1,2, \ldots, r]$. It is now known that infinitely many odd zeta values are irrational, without determining which ones (Rivoal [260, Ball and Rivoal [24]). In particular Zudilin 321] showed that at least one of $\zeta(5), \zeta(7), \zeta(9), \zeta(11)$ is irrational. See Fischler [117] for a survey of recent developments on irrationality of zeta values. The state of the art for showing transcendence of periods is surveyed by Waldschmidt 312 .

There is currently no effective way known to decide whether a given number is a period. One can certify a number is a period by directly exhibiting it as a $\overline{\mathbb{Q}}$-linear combination of values of known period integrals. However no properties of periods are currently known which would be useful in distinguishing them from nonperiods. Kontsevich and Zagier [186, Problem 3] raise the problem of exhibiting a single specific number that is provably not a period.

As mentioned in the Introduction it is conjectured that Euler's constant is not a period. The conjectures that $e$ and $\gamma$ are not periods appear in [186, Section 1.1]. We have already seen a number of different integral representations for Euler's constant, which however involve exponentials and/or logarithms, in addition to rational functions. These integrals do not resolve the question of whether $\gamma$ is a period.

Kontsevich and Zagier [186, Section 4.3] also suggest enlarging the class of periods to allow exponential functions in the integrals. They define an exponential period to be an absolutely convergent (multiple) integral of the product of an algebraic function with the exponential of an algebraic function, taken over a real semi-algebraic set, where all polynomials entering the definition of the algebraic functions and the semi-algebraic set have algebraic coefficients 32 The set of all such values forms a ring $\mathcal{E P}$, which we call the ring of exponential periods, and which contains the field $\overline{\mathbb{Q}}$ of all algebraic integers. This ring is countable, and is known to include the constant $e$, all values $\Gamma\left(\frac{p}{q}\right)$ at rational arguments, namely $\frac{p}{q} \in \mathbb{Q} \backslash \mathbb{Z}_{\leq 0}$, and the constant

$$
\sqrt{\pi}=\int_{-\infty}^{\infty} e^{-t^{2}} d t
$$

It also includes various values of period determinants for confluent hypergeometric functions studied by Terasoma [295, Theorem 2.3.3] and by Bloch and Esnault [35. Proposition 5.4]. Here we observe that:

(1) Euler's constant $\gamma \in \mathcal{E} \mathcal{P}$.

(2) The Euler-Gompertz constant $\delta \in \mathcal{E P}$.

M. Kontsevich observes that an integral representation certifying that $\gamma \in \mathcal{E P}$ is obtainable from (2.2.8) by substituting $-\log x=\int_{x}^{1} \frac{d y}{y}$ for $0<x<1$ and $\log x=\int_{1}^{x} \frac{d y}{y}$ for $x>1$, yielding

$$
\gamma=\int_{0}^{1} \int_{x}^{1} \frac{e^{-x}}{y} d y d x-\int_{1}^{\infty} \int_{1}^{x} \frac{e^{-x}}{y} d y d x .
$$

An integral representation certifying that $\delta \in \mathcal{E P}$ is given by (2.5.10), stating that

$$
\delta=\int_{0}^{\infty} \frac{e^{-t}}{1+t} d t
$$

It is not known whether $\delta$ is a period.

\footnotetext{
${ }^{32}$ The semi-algebraic set is cut out by a system of (real) polynomial inequalities $f_{j}\left(x_{1}, \ldots, x_{n}\right) \geq 0$, and it is supposed that the coefficients of the $f_{j}$ are all real algebraic numbers.
} 
3.15. Diophantine approximations to Euler's constant. Is Euler's constant rational or irrational? This is unknown.

One can empirically test this possibility by obtaining good rational approximations to Euler's constant. The early calculations of Euler's constant were based on Euler-Maclaurin summation. In 1872 Glaisher [128 reviewed earlier work on Euler's constant, which included his own 1871 calculation accurate to 100 places ([127]) and uncovered a mistake in an earlier calculation of Shanks. In 1878 the astronomer J. C. Adams 2 determined $\gamma$ to 263 places, again using Euler-Maclaurin summation, an enormous labor done by hand. In 1952 J. W. Wrench, Jr. 318, using a desk calculator, obtained $\gamma$ to 328 places. In 1962 Knuth [179] automated this approach and obtained Euler's constant to 1272 places with an electronic computer. However, this record did not last for long. In 1963 D. W. Sweeney 292 introduced a new method to compute Euler's constant, based on the integral representation

$$
\gamma=\sum_{k=1}^{\infty} \frac{(-1)^{k-1} n^{k}}{k ! k}-\int_{n}^{\infty} \frac{e^{-u}}{u} d u-\log n .
$$

By making a suitable choice of $n$ in this formula, Sweeney obtained 3566 digits of $\gamma$. The following year Beyer and Waterman 37. used a variant of this method to compute $\gamma$ to 7114 places, however only the initial 4879 digits of these turned out to be correct, as determined by later computations. In 1977 Brent 42 used a modification of this method to compute $\gamma$ and $e^{\gamma}$ to 20700 places. In 1980 Brent and McMillan [44] formulated new algorithms based on the identity

$$
\gamma=\frac{U(n)}{V(n)}-\frac{K_{0}(2 n)}{I_{0}(2 n)}
$$

whose right-hand side contains for $\nu=0$ the modified Bessel functions

$$
I_{\nu}(z):=\sum_{k=0}^{\infty} \frac{\left(\frac{z}{2}\right)^{\nu+2 k}}{k ! \Gamma(\nu+k+1)} \text { and } K_{0}(z):=-\left.\frac{\partial}{\partial \nu} I_{\nu}(z)\right|_{\nu=0},
$$

and

$$
\begin{aligned}
U(n) & :=\sum_{k=0}^{\infty}\left(\frac{n^{k}}{k !}\right)^{2}\left(H_{k}-\log n\right), \\
V(n) & :=I_{0}(2 n)=\sum_{k=0}^{\infty}\left(\frac{n^{k}}{k !}\right)^{2} .
\end{aligned}
$$

The second term $0<K_{0}(2 n) / I_{0}(2 n)<\pi e^{-4 n}$ can be made small by choosing $n$ large, hence $\gamma$ is approximated by $\frac{U(n)}{V(n)}$, choosing $n$ suitably (their Algorithm B1). With this algorithm they computed both $\gamma$ and $e^{\gamma}$ to 30000 places. They also determined the initial part of the ordinary continued fraction expansions of both $\gamma$ and $e^{\gamma}$, and established the following result.

Theorem 3.15.1 (Brent and McMillan 1980). (1) If $\gamma=\frac{m_{0}}{n_{0}}$ is rational, with $m_{0}, n_{0} \in \mathbb{Z}_{>0}$, then its denominator $n_{0}>10^{15000}$.

(2) If $e^{\gamma}=\frac{m_{1}}{n_{1}}$ is rational, with $m_{1}, n_{1} \in \mathbb{Z}_{>0}$, then its denominator $n_{1}>10^{15000}$.

Proof. We follow Brent [42. For any real number $\theta$, Theorem 17 of Khinchin [174] states, if $\frac{p_{n}}{q_{n}}$ is an ordinary continued fraction convergent for $\theta$, then $\left|q_{n} \theta-p_{n}\right| \leq$ $|q \theta-p|$ for all integers $p$ and $q$ with $0<|q| \leq q_{n}$. It therefore suffices to find a 
convergent $\frac{p_{n}}{q_{n}}$ with $q_{n}>10^{15000}$ such that $\theta \neq \frac{p_{n}}{q_{n}}$, and the latter inequality holds if $q_{n+1}$ exists.

The continued fraction expansion of $\gamma$ begins $\gamma=[0 ; 1,1,2,1,2,1,4,3,13,5,1,1,8,1,2,4,1,1,40,1,11,3,7,1,7,1,1,5,1,49,4, \ldots] ;$ see [274, Sequence A002852]. Brent and McMillan also computed various statistics for the initial part of this continued fraction, concerning the number of partial quotients of different sizes of the initial continued fractions of $\gamma$ and of $e^{\gamma}$, in order to test whether $\gamma$ behaves like a "random" real number. They found good agreement with the predicted limiting distribution of partial quotients for a random real number $\theta=\left[a_{0}, a_{1}, a_{2}, \ldots\right]$. It is a theorem of Kuz'min that the distribution of the $j$ th partial quotient $\operatorname{Prob}\left[a_{j}=k\right]$ rapidly approaches the Gauss-Kuz'min distribution

$$
p(k):=\log _{2}\left(1+\frac{1}{k}\right)-\log _{2}\left(1+\frac{1}{k+1}\right),
$$

as $j \rightarrow \infty$; see Khinchin [174, III.15].

Diophantine approximations to $\gamma$ can be extracted from various series expansions for $\gamma$ involving rational numbers. In 1910 Vacca 309] found the expansion

$$
\begin{aligned}
\gamma & =\sum_{k=1}^{\infty}(-1)^{k} \frac{\left\lfloor\log _{2} k\right\rfloor}{k} \\
& =\left(\frac{1}{2}-\frac{1}{3}\right)+2\left(\frac{1}{4}-\frac{1}{5}+\frac{1}{6}-\frac{1}{7}\right)+3\left(\frac{1}{8}-\frac{1}{9}+\cdots-\frac{1}{15}\right)+\cdots .
\end{aligned}
$$

Truncating this expansion at $k=2 m-1$ gives approximations $\frac{p_{m}}{q_{m}}$ to $\gamma$ satisfying

$$
0<\gamma-\frac{p_{m}}{q_{m}} \leq \frac{4(\log m+1)}{m}
$$

In 2010 Sondow 279 gave refinements of this expansion yielding approximations with a convergence rate $O\left(\frac{\log m}{m^{r}}\right)$ for an arbitrary but fixed $r \geq 2$. However approximations of these types fall far short of establishing irrationality of $\gamma$, since the denominators $q_{m}$ grow exponentially with $m$.

Much recent work on Diophantine approximation of explicit constants $\theta$ has focused on finding a series of rational approximations $\frac{u_{n}}{v_{n}}$ with the properties:

(1) Each sequence $u_{n}, v_{n}$ separately satisfies the same linear recurrence with coefficients that are polynomials in the ring $\mathbb{Z}[n]$, where $n$ is the recurrence parameter.

(2) The initial conditions for the recurrences $u_{n}, v_{n}$ are rational, so that both sequences consist of rational numbers.

(3) One has $\frac{u_{n}}{v_{n}} \rightarrow \theta$ as $n \rightarrow \infty$.

We give examples of such recurrences below; e.g. (3.15.1). For such sequences the power series $U(z)=\sum_{n=1}^{\infty} u_{n} z^{n}$ (resp. $V(z)=\sum_{n=1}^{\infty} v_{n} z^{n}$ ) will each (formally) satisfy a homogeneous linear differential equation $D F(z)=0$ in the $z$-variable, whose coefficients are polynomials in $\mathbb{Q}[z]$; i.e. the operator $D$ has the form

$$
D=\sum_{j=0}^{n} R_{j}(z) \frac{d^{j}}{d z^{j}} .
$$

We will refer to such a sequence of approximations satisfying (1), (2) as being of $H$-type and will call any real number obtainable as a limit of such approximations 
an elementary $H$-period. More generally, the ring $\mathcal{H} \mathcal{P}$ of $H$-periods will be the ring generated over $\overline{\mathbb{Q}}$ by elementary $H$-periods 33 The ring $\mathcal{H} \mathcal{P}$ is clearly countable, and its possible relations to rings of periods $\mathcal{P}$ or exponential periods $\mathcal{E} \mathcal{P}$ discussed in Section 3.14 remain to be determined.

It turns out to be useful to single out a subclass of such numbers, which are the set of such approximations for which the linear differential operators $D$ have a special property:

$G$-operator property. At some algebraic point $z_{0} \in \overline{\mathbb{Q}}$ the linear differential operator with coefficients in $\mathbb{C}[z]$ has a full rank set of a $G$-function solutions.

The notion of $G$-function is presented in detail in Bombieri [38, André [4], 8], and Dwork, Gerotto, and Sullivan [80. We give a definition of a subclass of $G$ functions obtained by specializing to the rational number case. (The general case allows algebraic number coefficients all drawn from a fixed number field $K$.) It is a function given by a power series $F(z)=\sum_{n=0}^{\infty} a_{n} z^{n}$, with rational $a_{n}$ such that

(1) There is a constant $C>0$ such that $\left|a_{n}\right| \leq C^{n}$, so that the power series $F(z)$ has a nonzero radius of convergence.

(2) There is a constant $C^{\prime}>0$ such that the least common multiple of the denominators of $a_{1}, \ldots, a_{n}$ is at most $\left(C^{\prime}\right)^{n}$.

(3) The $a_{n}=\frac{p_{n}}{q_{n}}$ are solutions of a linear recurrence having coefficients that are polynomials in the variable $n$ with integer coefficients.

The notion of a $G$-operator was formulated in André [4, IV. 5], [ㅁ, Section 3]. André's definition of a $G$-operator is given in another form, in terms of it satisfying the Galochkin condition (from Galochkin [123, also see Bombieri [38]), but this class of operators is known to coincide with those given by the definition above. The minimal differential operator satisfied by a $G$-function is known to be a $G$-operator in André's sense [6, Theorem 3.2], by a result of D. and G. Chudnovsky. Conversely, any $G$-operator in André's sense has a full rank set of $G$-function solutions at any algebraic point not a singular point of the operator ([6, Theorem 3.5]).

André [6, p. 719] has shown that $G$-operators, viewed in the complex domain, are of a very restricted type. They must be Fuchsian on the whole Riemann sphere $\mathbb{P}^{1}(\mathbb{C})$; i.e. they have only regular singular points, including the point $\infty$ (see also [8, Theorem 3.4.1]). A conjecture formulated in André [4, [6, p. 718] is that all $G$-operators should come from (arithmetical algebraic) "geometry", i.e. they each should be a product of factors of Picard-Fuchs operators, controlling the variation of cohomology in a parametrized family of algebraic varieties defined over $\overline{\mathbb{Q}}$.

We will say that a real number $\theta$ that is a limit of a convergent sequence of rational approximations $\frac{u_{n}}{v_{n}}$ with $u_{n}, v_{n}$ coefficients in the power series expansions of two (rational) $G$-functions is an elementary $G$-period. As above we obtain a ring $\mathcal{G P}$ of $G$-periods, defined as the ring generated over $\overline{\mathbb{Q}}$ by elementary $G$-periods. This ring has recently been shown in Fischler and Rivoal [118, Theorem 3] to coincide with the field $\operatorname{Frac}(\mathbf{G})$, in which $\operatorname{Frac}(\mathbf{G})$ is the fraction field of a certain ring $\mathbf{G}$ constructed from $G$-function values. The $\operatorname{ring} \mathbf{G}$ is defined to be the set of complex numbers $f(\alpha)$, where $\alpha \in \overline{\mathbb{Q}}$ and $f(z)$ is any branch of a (multivalued) analytic continuation of a $G$-function with coefficients defined over some number field which is nonsingular at $z=\alpha$. Fischler and Rivoal [118, Theorem 1] show that

\footnotetext{
${ }^{33}$ The name $H$-period is proposed by analogy with the more studied class of $G$-periods given below.
} 
the ring $\mathbf{G}$ coincides with the set of all complex numbers whose real and imaginary parts can each be written as $f(1)$, with $f(z)$ being some $G$-function with rational coefficients (as in the definition above) and whose power series expansion has radius of convergence exceeding 1 . Their result [118, Theorem 3] also establishes that the set of elementary $G$-periods is itself a ring containing all real algebraic numbers, which coincides with $\operatorname{Frac}(\mathbf{G}) \cap \mathbb{R}$. Again, possible relations of the field $\mathcal{G} \mathcal{P}$ with the Kontsevich-Zagier rings of periods $\mathcal{P}$ and extended periods $\mathcal{E} \mathcal{P}$ remain to be determined 34

As already mentioned in Section 3.14, a famous result of Apéry ([10], [11]) in 1979 established that the period $\zeta(3)$ is irrational. Apéry's result was obtained by finding rational approximations $\frac{p_{n}}{q_{n}}$ to $\zeta(3)$ which were generated by two different solutions $\left\{p_{n}, q_{n}: n \geq 1\right\}$ to the second order linear recurrence with polynomial coefficients:

$$
n^{3} u_{n}=\left(34 n^{3}-51 n^{2}+27 n-5\right) u_{n-1}-(n-1)^{3} u_{n-2}
$$

with initial conditions $p_{0}=0, p_{1}=6$ and $q_{0}=1, q_{1}=5$, respectively. Here $q_{n}$ are now called Apéry numbers, and are given by

$$
q_{n}=\sum_{k=0}^{n}\left(\begin{array}{c}
n+k \\
k
\end{array}\right)^{2}\left(\begin{array}{l}
n \\
k
\end{array}\right)^{2}
$$

while $p_{n}$ are given by

$$
p_{n}=\sum_{k=0}^{n}\left(\begin{array}{c}
n+k \\
k
\end{array}\right)^{2}\left(\begin{array}{l}
n \\
k
\end{array}\right)^{2}\left(\sum_{m=1}^{n} \frac{1}{m^{3}}+\sum_{m=1}^{k} \frac{(-1)^{m-1}}{2 m^{3}\left(\begin{array}{c}
n \\
m
\end{array}\right)\left(\begin{array}{c}
n+m \\
m
\end{array}\right)}\right) ;
$$

see Fischler [117, Section 1.2]). These approximations are closely spaced enough and good enough to imply that, for any $\epsilon>0$, and all $q \geq q(\epsilon)$ one has

$$
\left|\zeta(3)-\frac{p}{q}\right|>q^{-\theta+\epsilon} \text { for all } p \in \mathbb{Z},
$$

with exponent $\theta=13.41782$, which implies that $\zeta(3)$ is irrational. The method permits deriving families of approximations given by related recurrences for $\zeta(\mathrm{m})$ for all $m \geq 2$ (cf. Cohen [61]), but only for $m=2$ and 3 are they are good enough to prove irrationality. For each $m \geq 2$ the associated power series

$$
f(z)=\sum_{n \geq 0} p_{n} z^{n}, \quad g(z)=\sum_{n=0}^{\infty} q_{n} z^{n}
$$

will be solutions to a linear differential equation with polynomial coefficients, and are of $H$-type. For $m=2,3$ these approximations are known to be of $G$-type. A conceptually new proof of irrationality of $\zeta(3)$ using the same approximations, but connecting them with modular forms on the congruence subgroup $\Gamma_{1}(6)$ of $S L(2, \mathbb{Z})$ was presented in 1987 by Beukers [33.

Apéry's discovery was based on series acceleration transformations of the series $\zeta(3)=\sum_{n=1}^{\infty} \frac{1}{n^{3}}$. An initial example of Apéry of such accelerations is the identity

$$
\zeta(3)=\frac{5}{2} \sum_{n=1}^{\infty} \frac{(-1)^{n-1}}{n^{3}\left(\begin{array}{c}
2 n \\
n
\end{array}\right)}
$$

\footnotetext{
${ }^{34}$ The paper [118, Section 2.2] discusses the possibility that the equality $\mathbf{G}=\hat{\mathcal{P}}=\mathcal{P}\left[\frac{1}{\pi}\right]$ might hold, and it sketches an argument due to a reviewer that supports the conjecture that $\mathbf{G} \subseteq \hat{\mathcal{P}}$.
} 
see [249, Section 3]. The partial sums of this series converge at a rate $O\left(4^{-n}\right)$ to $\frac{2}{5} \zeta(3)$, but are by themselves insufficient to prove irrationality. Apéry's proof used further series acceleration transformations, which are described in [249, Section 4]. It is interesting to note that the identity 3.15.4) is a special case of identities in an 1890 memoir of A. A. Markoff [212 on series acceleration, as is explained in Kondratieva and Sadov [183]. In 1976 Gosper ([136], 137]) noted related identities, also found by series acceleration techniques, including

$$
\zeta(3)=\sum_{n=1}^{\infty} \frac{30 n-11}{16(2 n-1) n^{3}\left(\begin{array}{c}
2 n-1 \\
n
\end{array}\right)^{2}} .
$$

A new approach to Apéry's irrationality proof of $\zeta(3)$ was found by Nesterenko 231] in 1996, which used a relation of the Apéry approximations $p_{n}, q_{n}$ to Padé approximations to polylogarithms. Nesterenko also found a new continued fraction for $2 \zeta(3)$,

$$
2 \zeta(3)=2+\frac{1}{2+\frac{2}{4+\frac{1}{2+\frac{2}{4+\frac{6}{6+\frac{4}{5+\frac{9}{4+\cdots}}}}}}}
$$

Writing this as $2 \zeta(3)=b_{0}+\frac{a_{1}}{b_{1}+\cdots}$, the numerators $a_{n}$ grow quadratically in $n$ while the denominators $b_{n}$ grow linearly with $n$, and are given for $k \geq 0$ (excluding $\left.a_{1}\right)$ by

$$
\begin{array}{llll}
a_{4 k+1}=k(k+1), & b_{4 k+1}=2 k+2, & a_{4 k+2}=(k+1)(k+2), & b_{4 k+2}=2 k+4, \\
a_{4 k+3}=(k+1)^{2}, & b_{4 k+3}=2 k+3, & a_{4 k+4}=(k+2)^{2}, & b_{4 k+4}=2 k+2 .
\end{array}
$$

This result was obtained starting from Padé type simultaneous polynomial approximations to the logarithm, dilogarithm, and trilogarithm found by Gutnik [152]; see also the survey of Beukers [32. When specialized at the point $x=1$ these Padé approximations yield the sequences $q_{n},-2 p_{n}$. The continued fraction (3.15.5) was obtained from a Mellin-Barnes type integral for a special case of the Meijer $G$ function [216], a generalized hypergeometric function (see [87, Chapter V]). A survey of methods using Padé approximations to polylogarithms is given in Nesterenko 232. This approach has led to further results, including a new proof of irrationality of $\zeta(3)$ of Nesterenko 233 .

A natural question concerns whether $\gamma$ has rational approximations of $H$-type. A family of such rational approximations to $\gamma$ were found in 2007 through the combined efforts of A. I. Aptekarev, A. I. Bogolyubskii, D. V. Khristoforov, V. G. Lysov, and D. N. Tulyakov in the seven papers in the volume [12. Their result is stated in Aptekarev and Tulyakov [15] in the following slightly improved form. 
Theorem 3.15.2 (Aptekarev, Bogolyubskii, Khristoforov, Lysov, Tulyakov 2007). Euler's constant is approximated by a ratio of two rational solutions of the thirdorder recurrence relation with polynomial coefficients

$$
\begin{aligned}
(16 n-15) q_{n+1}= & \left(128 n^{3}+40 n^{2}-82 n-45\right) q_{n} \\
& -n^{2}\left(256 n^{3}-240 n^{2}+64 n-7\right) q_{n-1} \\
& +(16 n+1) n^{2}(n-1)^{2} q_{n-2} .
\end{aligned}
$$

The two solutions $\left\{p_{n}: n \geq 0\right\}$ and $\left\{q_{n}: n \geq 0\right\}$ are determined by the initial conditions

$$
p_{0}:=0, p_{1}:=2, p_{2}:=31
$$

and

$$
q_{0}:=1, q_{1}:=3, q_{2}:=50 .
$$

They have the following properties:

(1) Integrality. For $n \geq 0$,

$$
p_{n} \in \mathbb{Z}, q_{n} \in \mathbb{Z}
$$

(2) Denominator growth rate. For $n \geq 1$,

$$
q_{n}=(2 n) ! \frac{e^{\sqrt{2 n}}}{\sqrt[4]{n}}\left(\frac{1}{\sqrt{\pi}(4 e)^{3 / 8}}+O\left(\frac{1}{\sqrt{n}}\right)\right) .
$$

(3) Euler's constant approximation. For $n \geq 1$,

$$
\gamma-\frac{p_{n}}{q_{n}}=-2 \pi e^{-2 \sqrt{2 n}}\left(1+O\left(\frac{1}{\sqrt{n}}\right)\right) .
$$

The original result showed in place of (1) the weaker result that $q_{n} \in \mathbb{Z}$ and $D_{n} p_{n} \in$ $\mathbb{Z}$ with $D_{n}=$ l.c.m. $[1,2, \ldots, n]$. The integrality result for $p_{n}$ follows from a later result of Tulyakov 305, which finds a more complicated system of recurrences that these sequences satisfy, in terms of which the integrality of $p_{n}$ is manifest. These approximants clearly are of $H$-type.

The results in Theorem 3.15.2 are based on formulas derived from a family of multiple orthogonal polynomials in the sense of Aptekarev, Branquinho, and van Assche [14. These lead to exact integral formulas

$$
q_{n}=\int_{0}^{\infty} Q_{n}(x) e^{-x} d x
$$

and

$$
p_{n}-\gamma q_{n}=\int_{0}^{\infty} Q_{n}(x) e^{-x} \log x d x,
$$

in which $Q_{n}(x)$ are the family of polynomials (of degree $2 n$ ) given by

$$
Q_{n}(x)=\frac{1}{(n !)^{2}} \frac{e^{x}}{x-1}\left(\frac{d}{d x}\right)^{n} x^{n}\left(\frac{d}{d x}\right)^{n}(x-1)^{2 n+1} x^{n} e^{-x} .
$$

Note for $Q_{0}(x)=1$ that (3.15.6) becomes the integral formula (2.2.8) for $\Gamma^{\prime}(1)$. Also in 2009 Rivoal [261] found an alternative construction of these approximants that makes use of Padé acceleration methods for series similar to Euler's divergent series (2.5.1). His method applies more generally to numbers $\gamma+\log x$, where $x>0$ 
is rational. In 2010 Kh. and T. Hessami Pilehrood [162, Corollary 4] found closed forms for these approximants,

$$
q_{n}=\sum_{k=0}^{n}\left(\begin{array}{l}
n \\
k
\end{array}\right)^{2}(n+k) !, \quad p_{n}=\sum_{k=0}^{n}\left(\begin{array}{l}
n \\
k
\end{array}\right)^{2}(n+k) !\left(H_{n+k}+2 H_{n-k}-2 H_{k}\right),
$$

where the $H_{k}$ are harmonic numbers. These rational approximations $\frac{p_{n}}{q_{n}}$ thus are of a kind similar in appearance to Apéry's approximation sequence (3.15.2), (3.15.3) to $\zeta(3)$, and also satisfy a third order recurrence.

Recently, Kh. and T. Hessami Pilehrood [163, Theorem 1, Corollary 1] constructed an elegant sequence of rational approximations converging to Euler's constant, also analogous to the Apéry approximations to $\zeta(3)$, with slightly better convergence properties than those given in Theorem 3.15.2, and having a surprising connection with certain approximations to the Euler-Gompertz constant. They set

$$
q_{n}=\sum_{k=0}^{n}\left(\begin{array}{l}
n \\
k
\end{array}\right)^{2} k !, \quad p_{n}=\sum_{k=0}^{n}\left(\begin{array}{l}
n \\
k
\end{array}\right)^{2} k !\left(2 H_{n-k}-H_{k}\right) .
$$

The sequence $q_{n}$ satisfies the homogeneous second order recurrence

$$
q_{n+2}=2(n+2) q_{n+1}-(n+1)^{2} q_{n}
$$

and initial conditions $q_{0}=1, q_{1}=2$. The sequence $p_{n}$ satisfies the inhomogeneous second order recurrence

$$
p_{n+2}=2(n+2) p_{n+1}-(n+1)^{2} p_{n}-\frac{n}{n+2},
$$

with initial conditions $p_{0}=0, p_{1}=1$, and it satisfies a homogeneous third order recurrence.

Theorem 3.15.3 (Kh. and T. Hessami Pilehrood 2013). Let $\left(q_{n}\right)_{n \geq 0},\left(p_{n}\right)_{n \geq 0}$ be defined as above. Then $q_{n} \in \mathbb{Z}$ and $D_{n} p_{n} \in \mathbb{Z}$, where $D_{n}=1 . c . m .[1,2, \ldots, n]$, and for all $n \geq 1$,

$$
\gamma-\frac{p_{n}}{q_{n}}=-e^{-4 \sqrt{n}}\left(2 \pi+O\left(\frac{1}{\sqrt{n}}\right)\right) .
$$

Here the growth rate of the sequence $q_{n}$ is, for all $n \geq 1$,

$$
q_{n}=n ! \frac{e^{2 \sqrt{n}}}{\sqrt[4]{n}}\left(\frac{1}{2 \sqrt{\pi e}}+O\left(\frac{1}{\sqrt{n}}\right)\right) .
$$

These approximations converge to $\gamma$ from one side. The sequence $D_{n}$ needed to clear the denominator of $p_{n}$ is well known to have growth rate

$$
D_{n}=e^{n(1+o(1))},
$$

an asymptotic result that is equivalent in strength to the prime number theorem.

The Euler-Gompertz constant $\delta$ also has a convergent series of rational approximations with the same denominator sequence $q_{n}$ as the Euler constant approximations above. The new numerators $s_{n}$ satisfy the same recurrence (3.15.7) as the denominators $q_{n}$, i.e.

$$
s_{n+2}=2(n+2) s_{n+1}-(n+1)^{2} s_{n},
$$


but with initial conditions $s_{0}=0, s_{1}=1$. The $s_{n}$ are integers and are also given by the expression

$$
s_{n}=\sum_{k=1}^{n} a(k-1)(k+1)\left(\begin{array}{l}
n \\
k
\end{array}\right) \frac{(n-1) !}{(k-1) !},
$$

in which the function values $a(m)$ are

$$
a(m)=\sum_{k=0}^{m}(-1)^{k} k !
$$

see [274, Sequence A002793]. Here the $a(m)$ are the partial sums of Euler's divergent series discussed in Section 2.5. These approximations $\frac{s_{n}}{q_{n}}$ are exactly the partial quotients $\frac{s_{n}}{q_{n}}$ of the Laguerre continued fraction (2.5.19) for the Euler-Gompertz constant.

One has the following rate of convergence estimate for this sequence of rational approximations ([163, Theorem 2]).

Theorem 3.15.4 (Kh. and T. Hessami Pilehrood 2013). Let $\left(q_{n}\right)_{n \geq 0},\left(s_{n}\right)_{n \geq 0}$ be defined as above. Then $q_{n}, s_{n} \in \mathbb{Z}$, and for all $n \geq 1$, the approximations $s_{n} / q_{n}$ to the Euler-Gompertz constant satisfy

$$
\delta-\frac{s_{n}}{q_{n}}=e^{-4 \sqrt{n}}\left(2 \pi e+O\left(\frac{1}{\sqrt{n}}\right)\right) .
$$

The proof of Theorem 3.15 .4 relates these approximations to values of a MellinBarnes type integral

$$
I_{n}=(n !)^{2} \frac{1}{2 \pi i} \int_{c-i \infty}^{c+i \infty} \frac{\Gamma(s-n)^{2}}{\Gamma(s+1)} d s,
$$

where the vertical line of integration has $c>n$. The integral is expressed in terms of Whittaker function $W_{\kappa, \nu}(z)$ (a confluent hypergeometric function). The proof displays some identities involving the Euler-Gompertz constant and Whittaker function values, namely

$$
\delta=\frac{W_{-1 / 2,0}(1)}{W_{1 / 2,0}(1)}
$$

using

$$
W_{-1 / 2,0}(1)=\frac{\delta}{\sqrt{e}}, \quad W_{1 / 2,0}(1)=\frac{1}{\sqrt{e}} .
$$

Table 2 presents data on $\left(q_{n}, p_{n}, s_{n}\right)$ for small $n$ providing good approximations to $\gamma$ and $\delta$. Recall that $p_{n}$ are rational numbers, while $q_{n}, D_{n} p_{n}, s_{n}$ are integers, with $D_{n}=$ l.c.m. $[1,2, \ldots, n]$.

The approximations given in Theorems 3.15.3 and 3.15.4, certify that both $\gamma$ and $\delta$ are elementary $H$-periods, so that both $\gamma, \delta \in \mathcal{H P}$. These approximations are of insufficient quality to imply the irrationality of either Euler's constant or of the Euler-Gompertz constant. It remains a challenge to find a sequence of Diophantine approximations to $\gamma($ resp. $\delta$ ) that approach them sufficiently fast to certify irrationality.

One may ask whether $\gamma$ has Diophantine approximations of $G$-type. This is unknown. The current expectation is that this is not possible, i.e. that $\gamma \notin \mathcal{G P}$. Based on the observation of Rivoal and Fischler that $\mathcal{G P}=\operatorname{Frac}(\mathbf{G})$, and the 
TABLE 2. Approximants $p_{n} / q_{n}$ and $s_{n} / q_{n}$ to $\gamma$ and $\delta$, with $D_{n}=$ l.c.m. $[1,2, \ldots, n]$.

\begin{tabular}{|r|r|r|r|r|}
\hline$n$ & \multicolumn{1}{|r|}{$q_{n}$} & \multicolumn{1}{|c|}{$D_{n} p_{n}$} & \multicolumn{1}{|c|}{$D_{n}$} & \multicolumn{1}{|c|}{$s_{n}$} \\
\hline 0 & 1 & 0 & 1 & 0 \\
1 & 2 & 1 & 1 & 1 \\
2 & 7 & 8 & 2 & 4 \\
3 & 34 & 118 & 6 & 20 \\
4 & 209 & 1450 & 12 & 124 \\
5 & 1546 & 53584 & 60 & 920 \\
6 & 13327 & 461718 & 60 & 7940 \\
7 & 130922 & 31744896 & 420 & 78040 \\
8 & 1441729 & 699097494 & 840 & 859580 \\
9 & 17572114 & 25561222652 & 2520 & 10477880 \\
10 & 234662231 & 341343759982 & 2520 & 139931620 \\
\hline
\end{tabular}

the belief ([118, Section 2.2]) that the ring $\mathbf{G}$ may coincide with the KontsevichZagier ring $\hat{\mathcal{P}}$ of extended periods, this expectation amounts to a stronger form of Conjecture 1.0.2.

We conclude this section with recent results concerning $G$-type and $H$-type approximations to other constants considered by Euler. In 2010 Rivoal 263] showed that the periods $\Gamma\left(\frac{k}{n}\right)^{n}$, where $\frac{k}{n}>0$, can be approximated by sequences $\frac{p_{n}}{q_{n}}$ of rational numbers of the $G$-type, so are elementary $G$-periods. In another paper Rivoal 262 finds sequences of rational approximations showing that the individual numbers $\Gamma\left(\frac{k}{n}\right)$, which are not known to be periods for $0<k<n$, are elementary $H$-periods.

Finally we note that various authors have suggested other approaches to establishing the irrationality of Euler's constant; e.g. Sondow [276], 278] and Sondow and Zudilin [282.

3.16. Transcendence results related to Euler's constant. The first transcendence results for numbers involving Euler's constant came from the breakthrough of Shidlovskii [269], 270] in the 1950s and 1960s on transcendental values of $E$ functions, which is detailed in his book [271. The class of $E$-functions was introduced in 1929 by Siegel [272, p. 223], consisting of those analytic functions $F(z)=\sum_{n=0}^{\infty} c_{n} \frac{z^{n}}{n !}$ whose power series expansions have the following properties:

(1) The coefficients $c_{n}$ belong to a fixed algebraic number field $K$ (a finite extension of $\mathbb{Q}$ ). For each $\epsilon>0$, the maximum of the absolute values of the algebraic conjugates of $c_{n}$ is bounded by $O\left(n^{n \epsilon}\right)$ as $n \rightarrow \infty$.

(2) For each $\epsilon>0$ such that there is a sequence of integers $q_{0}, q_{1}, q_{2}, \ldots$ such that $q_{n} c_{n}$ is an algebraic integer, with $q_{n}=O\left(n^{n \epsilon}\right)$ as $n \rightarrow \infty$.

(3) The function $F(z)$ satisfies a (nontrivial) linear differential equation

$$
\sum_{j=0}^{n} R_{j}(z) \frac{d^{j}}{d z^{j}} y(z)=0,
$$

whose coefficients are polynomials $R_{j}(z) \in K_{1}[z]$, where $K_{1}$ is some algebraic number field 35

\footnotetext{
${ }^{35}$ This condition on $K_{1}$ could be weakened to require only that the coefficients are in $\mathbb{C}[z]$.
} 
Each $E$-function is an entire function of the complex variable $z$. The set of all $E$ functions forms a ring $\mathbf{E}$ (under $(+, \times)$ ) which is also closed under differentiation, and this ring is an algebra over $\overline{\mathbb{Q}}$.

We also define a subclass of $E$-functions called $E^{*}$-functions in parallel with the class of $G$-functions, following Shidlovskii [271, Chapter 13]. An analytic function $F(z)=\sum_{n=1}^{\infty} c_{n} \frac{z^{n}}{n !}$ is an $E^{*}$-function if it has the following properties:

(1) $F(z)$ is an $E$-function.

(2) There is some constant $C>0$ such that the maximum of the absolute values of the algebraic conjugates of $c_{n}$ is bounded by $O\left(C^{n}\right)$ as $n \rightarrow \infty$.

(3) There is some constant $C^{\prime}>0$ such that there is a sequence of integers $q_{0}, q_{1}, q_{2}, \ldots$ such that each $q_{n} c_{n}$ is an algebraic integer, and with $q_{n}=$ $O\left(\left(C^{\prime}\right)^{n}\right)$ as $n \rightarrow \infty$.

This definition narrows the growth rate conditions on the coefficients compared to Siegel's conditions. The set of all $E^{*}$-functions forms a ring $\mathbf{E}^{*}$ closed under differentiation which is also an algebra over $\overline{\mathbb{Q}}$. Certainly $\mathbf{E}^{*} \subseteq \mathbf{E}$, and it is conjectured that the two rings are equal [271, p. 407]. Results of André ([6], 7]) apply to the class $\mathbf{E}^{*}$.

Siegel $[272$, Teil I] originally applied his method to prove transcendence of algebraic values of the Bessel function $J_{0}(\alpha)$ for algebraic $\alpha$ where $J_{0}(\alpha) \neq 0$. In formalizing his method in 1949, Siegel [273] first converted an $n$th order differential operator to a linear system of first order linear differential equations

$$
\frac{d y_{i}}{d x}=\sum_{j=1}^{n} R_{i j}(x) y_{j}, 1 \leq i \leq n,
$$

where the functions $R_{i, j}(x)$ are rational functions. In order for his proofs to apply, he required this system to satisfy an extra condition which he called normal. Siegel was able to verify normality in a few cases, including the exponential function and certain Bessel functions. A great breakthrough of Shidlovskii [269] in 1959 permitted the method to prove transcendence of solution values for all cases where the obvious necessary conditions are satisfied. The Siegel-Shidlovskii theorem states that if the $E_{i}(x)$ are algebraically independent functions over the rational function field $K(x)$, then at any nonzero algebraic number not a pole of any function $R_{i j}(x)$, the values $E_{j}(\alpha)$ are algebraically independent. For treatments of these results, see Shidlovskii [271] and Feldman and Nesterenko [114, Chapter 5].

More recent progress on $E$-functions includes a study in 1988 of the Siegel normality condition by Beukers, Brownawell, and Heckman [34] which obtains effective measures of algebraic independence in some cases. In 2000 André [6, Theorem 4.3] (see also [8]) established that for each $E^{*}$-function the minimal order linear differential equation with $\mathbb{C}(z)$ coefficients that it satisfies has singular points only at $z=0$ and $z=\infty$; that is, it has a basis of $n$ independent holomorphic solutions at all other points. Furthermore $z=0$ is necessarily a regular singular point and $\infty$ is an irregular singular point of a restricted type. This result goes a long way towards explaining why all the known $E^{*}$-functions are of hypergeometric type. André 7 ] applied this characterization to give a new proof of the Siegel-Shidlovskii theorem. Finally, we remark that although transcendence results are usually associated to $E$-functions and irrationality results to $G$-functions, in 1996 André [5] obtained transcendence results for certain $G$-function values. 
Returning to Euler's constant, in 1968 Mahler [209] applied Shidlovskii's methods to certain Bessel functions. For example

$$
J_{0}(z)=\sum_{n=0}^{\infty} \frac{(-1)^{n}}{(n !)^{2}}\left(\frac{z}{2}\right)^{2 n}
$$

is a Bessel function of the first kind, and

$$
Y_{0}(z)=\frac{2}{\pi}\left(\log \left(\frac{z}{2}\right)+\gamma\right) J_{0}(z)+\frac{2}{\pi}\left(\sum_{n=1}^{\infty}(-1)^{n-1} \frac{H_{n}}{(n !)^{2}}\left(\frac{z^{2}}{4}\right)^{n}\right)
$$

is a Bessel function of the second kind; see [1, (9.1.13)]. Here $J_{0}(z)$ is an $E^{*}$ function, but $Y_{0}(z)$, which contains Euler's constant in its expansion (3.16.1), is not, because it has a logarithmic singularity at $x=0$. However the modified function

$$
\tilde{Y}_{0}(z):=\frac{\pi}{2} Y_{0}(z)-\left(\log \left(\frac{z}{2}\right)+\gamma\right) J_{0}(z)=\sum_{n=1}^{\infty}(-1)^{n-1} \frac{H_{n}}{(n !)^{2}}\left(\frac{z^{2}}{4}\right)^{n}
$$

is an $E^{*}$-function, and Mahler uses this fact. He noted the following special case of his general results.

Theorem 3.16.1 (Mahler 1968). The number

$$
\frac{\pi}{2} \frac{Y_{0}(2)}{J_{0}(2)}-\gamma
$$

is transcendental.

We have $J_{0}(2)=\sum_{n=0}^{\infty} \frac{(-1)^{n}}{(n !)^{2}}$ and $\frac{\pi}{2} Y_{0}(2)=\gamma J_{0}(2)+\sum_{n=1}^{\infty}(-1)^{n-1} \frac{H_{n}}{(n !)^{2}}$, from which it follows that Mahler's transcendental number is

$$
\frac{\pi}{2} \frac{Y_{0}(2)}{J_{0}(2)}-\gamma=\frac{\sum_{n=1}^{\infty} \frac{(-1)^{n-1} H_{n}}{(n !)^{2}}}{\sum_{n=0}^{\infty} \frac{(-1)^{n}}{(n !)^{2}}}
$$

More recent transcendence results apply to Euler's constant along with other constants. In 2012 Rivoal 264 proved a result which, as a special case, implies the transcendence of at least one of the Euler constant $\gamma$ and the Euler-Gompertz constant $\delta$. It uses the Shidlovskii method and improves on an observation of Aptekarev [13] that at least one of $\gamma$ and $\delta$ must be irrational. This transcendence of at least one of $\gamma$ and $\delta$ was also established about the same time by Kh. and T. Hessami Pilehrood [163, Corollary 3].

Theorem 3.16.2 (Rivoal 2012). Euler's constant $\gamma$ and the Euler-Gompertz constant

together have the following properties:

$$
\delta:=\int_{0}^{\infty} \frac{e^{-w}}{1+w} d w=\int_{0}^{1} \frac{d v}{1-\log v}
$$

(1) Simultaneous Diophantine approximation. One cannot approximate the pair $(\gamma, \delta)$ very well with rationals $\left(\frac{p}{q}, \frac{r}{q}\right)$ having the same denominator. For each $\epsilon>0$ there is a constant $C(\epsilon)>0$ such that for all integers $p, q, r$ with $q \neq 0$, there holds

$$
\left|\gamma-\frac{p}{q}\right|+\left|\delta-\frac{r}{q}\right| \geq \frac{C(\epsilon)}{H^{3+\epsilon}}
$$

where $H=\max (|p|,|q|,|r|)$. 
(2) Transcendence. The transcendence degree of the field $\mathbb{Q}(e, \gamma, \delta)$ generated by $e, \gamma$, and $\delta$ over the rational numbers $\mathbb{Q}$ is at least two.

Proof. This follows from a much more general result of Rivoal [264, Theorem 1], which concerns values of the function

$$
\mathcal{G}_{\alpha}(z):=z^{-\alpha} \int_{0}^{\infty}(t+z)^{\alpha-1} e^{-t} d t .
$$

Here we specialize to the case $\alpha=0$, and on taking $z=1$ we have

$$
\mathcal{G}_{0}(1)=\int_{0}^{\infty} \frac{e^{-t}}{t+1} d t=\delta
$$

using Hardy's integral (2.5.10). The function $\mathcal{G}_{0}(z)$ satisfies a linear differential equation, but is not an $E$-function in the sense of transcendence theory. Rivoal makes use of the identity

$$
\gamma+\log z=-e^{-z} \mathcal{G}_{0}(z)-\mathcal{E}(-z),
$$

valid for $z \in \mathbb{C} \backslash \mathbb{R}_{\leq 0}$, where the entire function

$$
\mathcal{E}(z):=\sum_{n=1}^{\infty} \frac{z^{n}}{n \cdot n !},
$$

is an $E^{*}$-function.

Result (i) follows from the assertion of Theorem 1 (ii) of Rivoal 264, specialized to parameter values $\alpha=0$ and $z=1$. This result uses explicit Hermite-Padé approximants to the $E^{*}$-functions $1, e^{z}, \mathcal{E}_{\alpha}(z)$, where $\mathcal{E}_{\alpha}(z)=\sum_{m=0}^{\infty} \frac{z^{m}}{m !(m+\alpha+1)}$, with $\alpha \neq-1,-2,-3, \ldots$ Here $\mathcal{E}(z)$ corresponds to $\alpha=-1$ with the divergent constant term dropped from the power series expansion.

Result (ii) follows from the assertion of Theorem 2 (ii) of Rivoal [264, which asserts for (complex) algebraic numbers $z \notin(-\infty, 0]$ that the field generated over $\mathbb{Q}$ by the three numbers $e^{z}, \gamma+\log z, \mathcal{G}_{0}(z)$ has transcendence degree at least two. It makes use of (3.16.2), which can be rewritten as the integral identity

$$
-\gamma=\log z+z \int_{0}^{1} e^{-t z} \log t d t+e^{-z} \int_{0}^{\infty} \frac{e^{-t}}{t+z} d t .
$$

It uses the specialization $z=1$, which essentially gives Hardy's identity (2.5.11) divided by $e$, which we may rewrite as

$$
-\mathcal{E}(-1)=\gamma+\frac{\delta}{e}
$$

The result then follows using Shidlovskii's Second Fundamental Theorem 271, p. 123].

Rivoal 264 remarks that the transcendence result Theorem 3.16.2(2) is implicit in the approach that Mahler [209] formulated in 1968. At the end of his paper Mahler [209, p. 173] states without details that his method extends to show that for rational $\nu_{0}>0$ and nonzero algebraic numbers $\alpha$, the integrals

$$
I_{k}\left(\nu_{0}, \alpha\right):=\int_{0}^{1} x^{\nu_{0}-1}(\log x)^{k} e^{-\alpha x} d x, k=0,1,2, \ldots
$$


are algebraically independent transcendental numbers. Choosing $\nu_{0}=1, \alpha=1$ and $k=0,1$, we have

$$
I_{0}(1,1):=\int_{0}^{1} e^{-x} d x=1-\frac{1}{e}
$$

and, using (2.5.9),

$I_{1}(1,1)=\int_{0}^{1}(\log x) e^{-x} d x=\int_{0}^{\infty}(\log x) e^{-x} d x-\frac{1}{e} \int_{0}^{\infty} \log (x+1) e^{-x} d x=-\gamma-\frac{\delta}{e}$.

Mahler's statement asserts that these are algebraically independent transcendental numbers. This assertion implies that $\mathbb{Q}(\gamma, \delta, e)$ has transcendence degree at least 2 over $\mathbb{Q}$.

We next present recent results which concern the transcendence of collections of generalized Euler constants discussed in Section 3.8. These results are applications of Baker's results on linear forms in logarithms and allow the possibility of one exceptional algebraic value. Recall that the Euler-Lehmer constants, for $0 \leq h<k$ studied by Lehmer [201, are defined by

$$
\gamma(h, k):=\lim _{x \rightarrow \infty}\left(\sum_{\substack{0 \leq n<x \\ n \equiv h(\bmod k)}} \frac{1}{n}-\frac{\log x}{k}\right) .
$$

In 2010 M. R. Murty and N. Saradha [228, Theorem 1] obtained the following result.

Theorem 3.16.3 (Murty and Saradha 2010). In the infinite list of Euler-Lehmer constants

$$
\{\gamma(h, k): 1 \leq h<k, \text { for all } k \geq 2\},
$$

at most one value is an algebraic number. If Euler's constant $\gamma$ is an algebraic number, then only the number $\gamma(2,4)=\frac{1}{4} \gamma$ in the above list is algebraic.

Their proof uses the fact that a large set of linear combinations of $\gamma(h, k)$ are equal to logarithms of integers. Then Baker's bounds for linear forms in logarithms of algebraic numbers are applied to derive a contradiction from the assumption that the set contains at least two algebraic numbers. An immediate consequence of Theorem 3.16.3 is the fact that over all rational numbers $0<x \leq 1$ at least one of $\Gamma(x), \Gamma^{\prime}(x)$ is transcendental, with at most one possible exceptional $x$ [228, Corollary]; see also Murty and Saradha [227.

In a related direction Murty and Zaytseva [229, Theorem 4] obtained a corresponding transcendence result for the generalized Euler constants $\gamma(\Omega)$ associated to a finite set $\Omega$ of primes that were introduced by Diamond and Ford [71]. Recall from Section 3.8 that these constants are obtained by setting $\zeta_{\Omega}(s)=\prod_{p \in \Omega}\left(1-p^{-s}\right) \zeta(s)$ and defining $\gamma(\Omega)$ as the constant term in its Laurent expansion around $s=1$, writing

$$
\zeta_{\Omega}(s)=\frac{D(\Omega)}{s-1}+\gamma(\Omega)+\sum_{n=1}^{\infty} \gamma_{n}(\Omega)(s-1)^{n} .
$$

In particular $\gamma(\emptyset)=\gamma$.

Theorem 3.16.4 (Murty and Zaytseva 2013). In the infinite list of Diamond-Ford generalized Euler constants $\gamma(\Omega)$, where $\Omega$ runs over all finite subsets of primes (including the empty set), all numbers are transcendental with at most one exception. 
The constants $\gamma(\Omega)$ can be expressed as finite linear combinations of EulerLehmer numbers (cf. (3.8.1) ) so this result is closely related to the previous theorem. This result is also proved by contradiction, using Baker's results on linear forms in logarithms. If $\gamma$ were algebraic, then $\gamma(\emptyset)$ would be the unique exceptional value. However one expects there to be no algebraic value in the list.

\section{Concluding REmarks}

Euler's constant appears sui generis. Despite three centuries of effort, it seems unrelated to other constants in any simple way.

A first consequence arising from this is the appearance of Euler's constant in ostensibly unrelated fields of mathematics may signal some hidden relationship between these fields. A striking example is the analogy between results concerning the structure of factorizations of random integers and cycle structure of random permutations. These results are given for restricted factorizations in Sections 3.5 and [3.6, and for restricted cycle structure of permutations in Sections 3.10 and 3.11. respectively. Results in these two fields were initially proved separately and were later unified in limiting probability models developed by Arratia, Barbour, and Tavaré [17. These models account not only for the main parts of the probability distribution but also for tail distributions, where there are large deviations results in which Euler's constant appears. On a technical level an explanation for the parallel results in the two subjects was offered by Panario and Richmond 242 at the level of asymptotic analysis of generating functions of a particular form. This research development is an example of one of Euler's research methods detailed in Section 2.6. that of looking for a general scheme uniting two special problems.

In a second direction, the study of Euler's constant and multiple zeta values can now be viewed in a larger context of rings of periods connected with algebraic integrals. The sui generis nature of $\gamma$ is sharply formulated in the conjecture that it is not a Kontsevich-Zagier period. On the other hand, it is known to belong to the larger ring of exponential periods $\mathcal{E P}$, as described in Section 3.14. This research development illustrates another of Euler's research methods, to keep searching for a fuller understanding of any problem already solved.

In a third direction, one may ask for an explanation of the appearance of Euler's constant in various sieve method formulas. Here Friedlander and Iwaniec [121, p. 239] comment that these appearances stem from two distinct sources. The first is through Mertens's product theorem (Theorem 3.3.2). The second is via differentialdifference equations of the types treated in Sections 3.5 and 3.6.

In a fourth direction, there remain mysteries about the appearance of Euler's constant in number theory in the gamma and zeta function.

Observation. Euler's constant $\gamma$ appears in the Laurent expansion of the Riemann zeta function at $s=1$; here the zeta function is a function encoding the properties of the finite primes. Euler's constant also appears in the Taylor expansion of the gamma function at $s=1$; here the gamma function encodes properties of the (so-called) "infinite prime" or "real prime" (Archimedean place).

The analogy between the real prime and the finite primes traces back to Ostrowski's work in the period 1913-1917 which classified all absolute values on $\mathbb{Q}$ (Ostrowski's Theorem). Such analogies have been pursued for a century; a comprehensive treatment of such analogies is given in Haran [153. 
A mild mystery is to give a conceptual explanation for this occurrence of Euler's constant in two apparently different contexts: one associated to the finite primes and the other associated to the real prime (Archimedean place). There is a known connection between these two contexts, given by the product formula for rational numbers over all places, finite and infinite. It says

$$
\prod_{p \leq \infty}|r|_{p}=1, \quad r \in \mathbb{Q}
$$

in which $|\cdot|_{p}$ denotes the $p$-adic valuation, normalized with $|p|_{p}=\frac{1}{p}$ and $|x|_{\infty}$ being the real absolute value. Does this relation explain the common appearance? On closer examination there appears to be a mismatch between these two occurrences, visible in the functional equation of the Riemann zeta function. The functional equation can be written

$$
\hat{\zeta}(s)=\hat{\zeta}(1-s)
$$

in which appears the completed zeta function

$$
\hat{\zeta}(s):=\pi^{-\frac{s}{2}} \Gamma\left(\frac{s}{2}\right) \zeta(s)=\pi^{-\frac{s}{2}} \Gamma\left(\frac{s}{2}\right) \prod_{p}\left(1-\frac{1}{p^{s}}\right)^{-1} .
$$

The completed zeta function is given as an Euler product over all "primes", both the finite primes and the Archimedean Euler factor $\pi^{-\frac{s}{2}} \Gamma\left(\frac{s}{2}\right)$. In this formula evaluation at the point $s=1$ for the Riemann zeta function corresponds to evaluation of the gamma function at the shifted point $s^{\prime}=\frac{s}{2}=\frac{1}{2}$. The mismatch is that for the gamma function, Euler's constant most naturally appears in connection with its derivative at the point $s^{\prime}=1$, rather than at the point $s^{\prime}=\frac{1}{2}$, cf (3.1.1). Is there a conceptual explanation of this mismatch? Theorem 3.1.1 associates Euler's constant with the digamma function at $s=\frac{1}{2}$, but in that case an extra factor of $\log 2$ appears. Perhaps this mismatch can be resolved by considering an asymmetric functional equation for the function $\Gamma(s) \zeta(s)$. Here one has, valid for $\operatorname{Re}(s)>1$, the integral formula

$$
\Gamma(s) \zeta(s)=\int_{0}^{\infty} \frac{1}{e^{x}-1} x^{s-1} d x .
$$

One of Riemann's proofs of the functional equation for $\zeta(s)$ is based on this integral.

Another mystery concerns whether there may exist an interesting arithmetical dynamical interpretation of Euler's constant, different from that given in Section 3.12 The logarithmic derivative of the Archimedean factor $\pi^{-\frac{s}{2}} \Gamma\left(\frac{s}{2}\right)$ at the value $s=2$ (that is, $s^{\prime}=1$ ) produces the value $-g / 2$, where

$$
g:=\gamma+\log \pi .
$$

The constant $g$ appears elsewhere in the "explicit formulas" of prime number theory, as a contribution at the (special) point $x=1$ in the Fourier test function space; cf. [39, p. 280]. Perhaps one should search for a dynamical system whose entropy is the constant $-g:=-\gamma-\log \pi \approx-1.721945$.

In a fifth direction, Euler's constant can be viewed as a value emerging from regularizing the zeta function at $s=1$, identified with the constant term in its Laurent series expansion at $s=1$. There are a number of different proposals for "renormalizing" multiple zeta values, including the value of " $\zeta(1)$ ", with the intent to preserve other algebraic structures. This is an active area of current research. Various alternatives are discussed in Ihara, Kaneko, and Zagier 171, Guo and 
Zhang [150, 151, Guo, Paycha, Xie, and Zhang [149, and Manchon and Paycha 211. Some of these authors (for example [171]) prefer to assign the renormalized value 0 to $\zeta(1)$, to be obtained by further subtracting off Euler's constant.

This direction generalizes to algebraic number fields. For background we refer to Neukirch [235, Chapter VII. Section 5]. The Dedekind zeta function $\zeta_{K}(s)$ of a number field $K$ is given by

$$
\zeta_{K}(s)=\sum_{A} \frac{1}{N_{K / \mathbb{Q}}(A)^{s}},
$$

where $A$ runs over all the ideals in the ring of integers $O_{K}$ of the number field, and $N_{K / \mathbb{Q}}$ is the norm function. This function analytically continues to a meromorphic function on $\mathbb{C}$ which has a simple pole at $s=1$, with Laurent expansion

$$
\zeta_{K}(s)=\frac{\alpha_{-1}(K)}{s-1}+\alpha_{0}(K)+\sum_{j=1}^{\infty} \alpha_{j}(K)(s-1)^{j} .
$$

The term $\alpha_{-1}(K)$ encodes arithmetic information about the field $K$, given in the analytic class number formula

$$
\operatorname{Re}_{s=1}\left(\zeta_{K}(s)\right):=\alpha_{-1}(K)=\frac{h_{K} R_{K}}{e^{g_{K}}},
$$

in which $h_{K}$ is the (narrow) class number of $K, R_{K}$ is the regulator of $K$ (the log-covolume of the unit group of $K$ ), and $g_{K}$ is the genus of $K$, given by

$$
g_{K}:=\log \frac{w_{K}\left|d_{K}\right|^{1 / 2}}{2^{r_{1}}(2 \pi)^{r_{2}}},
$$

in which $w_{K}$ is the number of roots of unity in $K, d_{K}$ is the absolute discriminant of $K, r_{1}$ and $r_{2}$ denote the number of real (resp. complex) Galois conjugate fields to $K$, inside $\overline{\mathbb{Q}}$. The constant term $\alpha_{0}(K)$ is then an analogue of Euler's constant for the number field $K$, since $\alpha_{0}(\mathbb{Q})=\gamma$. A refined notion studies the constant term of a zeta function attached to an individual ideal class $\mathcal{C}$ of a number field $K$. Such a partial zeta function has the form

$$
\zeta(\mathcal{C}, s):=\sum_{A \in \mathcal{C}} \frac{1}{N_{K / \mathbb{Q}}(A)^{s}},
$$

where the sum runs over all integral ideals $A$ of the number field belonging to the ideal class $\mathcal{C}$. The Dedekind zeta function is the sum of ideal class zeta functions over all $h_{K}$ ideal classes. These partial zeta functions $\zeta(\mathcal{C}, s)$ extend to meromorphic functions of $s$ on $\mathbb{C}$ with only singularity a simple pole at $s=1$ having a residue term that is independent of $\mathcal{C}$; i.e. $\alpha_{-1}(\mathcal{C}, K)=\frac{1}{h_{K}} \alpha_{-1}(K)$ 235, Theorem VII (5.9)].

For imaginary quadratic fields the constant term $a_{0}(\mathcal{C}, K)$ in the Laurent expansion at $s=1$ of an ideal class zeta function can be expressed in terms of a modular form using the first Kronecker limit formula; cf. Kronecker [187]. This limit formula concerns the real-analytic Eisenstein series, for $\tau=x+i y$ with $y>0$ and $s \in \mathbb{C}$

$$
E(\tau, s)=\sum_{(m, n) \neq(0,0)} \frac{y^{s}}{|m \tau+n|^{2 s}},
$$


which for fixed $\tau$ analytically continues to a meromorphic function of $s \in \mathbb{C}$ and has a simple pole at $s=1$ with residue $\pi$ and Laurent expansion

$$
E(\tau, s)=\frac{\pi}{s-1}+c_{0}(\tau)+\sum_{j=1}^{\infty} c_{n}(\tau)(s-1)^{n} .
$$

The first Kronecker limit formula expresses the constant term $c_{0}(\tau)$ as

$$
c_{0}(\tau)=2 \pi(\gamma-\log 2)-\log \left(\sqrt{y}|\eta(\tau)|^{2}\right),
$$

in which $\eta(\tau)$ is the Dedekind eta function, a modular form given by

$$
\eta(\tau)=q^{1 / 24} \prod_{n=1}^{\infty}\left(1-q^{n}\right), \text { with } q=e^{2 \pi i \tau} ;
$$

see 319, Section 2]. In 1923 Herglotz 160 found an analogous formula for the constant term in the Laurent series expansion at $s=1$ of $\zeta(\mathcal{C}, s)$ for an ideal class $\mathcal{C}$ of a real quadratic field, which involved Dedekind sums and an auxiliary transcendental function. In 1975 by Zagier 319] obtained another formula for this case which is expressed using continued fractions and an auxiliary function.

In 2006 Ihara [172] studied another quantity extracted from the Laurent expansion (4.1) of the Dedekind zeta function of a number field at $s=1$, given by

$$
\gamma(K):=\frac{\alpha_{0}(K)}{\alpha_{-1}(K)}
$$

which he termed the Euler-Kronecker constant of the number field $K$. Here $\gamma(K)$ represents a scaled form of the constant term, and it generalizes Euler's constant since $\gamma(\mathbb{Q})=\gamma$. Motivated by the function field case, Ihara hopes that $\gamma(K)$ encodes interesting information of a different type on the field $K$, especially as $K$ varies. Under the assumption of the generalized Riemann hypothesis, Ihara obtained upper and lower bounds

$$
-c_{1} \log D_{K} \leq \gamma(K) \leq c_{2} \log \log D_{K}
$$

where $D_{K}$ is the (absolute value of the) discriminant of $K$ and $c_{1}, c_{2}>0$ are absolute constants. Further unconditional upper bounds on $\gamma(K)$ were obtained by Murty [230] and lower bounds were given by Badzyan [22].

Finally, ongoing attempts to place Euler's constant in a larger context have continued in many directions. These include studies of new special functions involving Euler's constant, such as the generalized Euler-constant function $\gamma(z)$ proposed by Sondow and Hadjicostas [280]. Two other striking formulas, found in 2005 by Sondow [277], are the double integrals

$$
\int_{0}^{1} \int_{0}^{1} \frac{1-x}{(1-x y)(-\log x y)} d x d y=\gamma
$$

and

$$
\int_{0}^{1} \int_{0}^{1} \frac{1-x}{(1+x y)(-\log x y)} d x d y=\log \frac{4}{\pi}
$$

Guillera and Sondow [148 then found a common integral generalization with a complex parameter that unifies these formulas with double integrals of Beukers 31 . for $\zeta(2)$ and $\zeta(3)$; see (3.14.1).

Many other generalizations and variants of Euler's constant have been proposed. These include a $p$-adic analogue of Euler's constant introduced by J. Diamond [72] in 1977 (see also Koblitz [181] and Cohen [62, Section 11.5]) and $q$-analogues of 
Euler's constant formulated by Kurokawa and Wakayama [188] in 2004. A topic of perennial interest is the search for new expansions and representations for Euler's constant. For example 1917 work of Ramanujan 254] was extended in 1994 by Brent [43. Some new expansions involve accelerated convergence of known expansions; e.g. Elsner [85, K. and T. Hessami Pilehrood [161, and Prévost [250]. Recent developments of the Dickman function led to the study of new constants involving Euler's constant and polylogarithms; cf. Broadhurst [48] and Soundararajan [284].

All these studies, investigations of irrationality, connections with the Riemann hypothesis, possible interpretation as a (non-)period, and formulating generalizations of Euler's constant, may together lead to a better understanding of the place of Euler's constant $\gamma$ in mathematics.

\section{ACKNOWLEDGMEnTS}

This paper started with a talk on Euler's constant given in 2009 in the "What is...?" seminar run by Li-zhen Ji at the University of Michigan. I am grateful to Jordan Bell for providing Latin translations of Euler's work in Sections 2.1-2.3 and for pointing out relevant Euler papers E55, E125, E629, and I thank David Pengelley for corrections and critical comments on Euler's work in Section 2. I thank Sylvie Paycha for comments on dimensional regularization in Section 3.2; Doug Hensley, Pieter Moree, K. Soundararajan, and Gerald Tenenbaum for remarks on Dickman's function in Section 3.5; Andrew Granville for extensive comments on the anatomy of integers ([141]), affecting Sections 3.5, 3.6, and 3.9-3.11; Tony Bloch for pointing out work of Cohen and Newman described in Section 3.13; Maxim Kontsevich for comments on rings of periods in Section 3.14; and Stephane Fischler, Tanguy Rivoal, and Wadim Zudilin for comments on Sections 3.15 and 3.16. I thank Daniel Fiorilli, Yusheng Luo, and Rob Rhoades for corrections. I am especially grateful to Juan Arias de Reyna for detailed checking, detecting many misprints and errors, and supplying additional references. Finally, I thank the reviewers for many historical and mathematical suggestions and corrections.

\section{About the Author}

Jeffrey C. Lagarias completed his doctorate in 1974 at MIT under the supervision of Harold M. Stark, with a thesis in algebraic number theory. He worked at Bell Laboratories and its descendants until 2003, before joining the faculty at the University of Michigan.

\section{REFERENCES}

[1] M. Abramowitz and I. Stegun, Eds., Handbook of Mathematical Functions with Formulas, Graphs and Mathematical Tables, U.S. Govt. Printing Office, June 1964. (Reprint: Dover.) $[2.5,3.1 .3 .16]$

[2] J. C. Adams, Note on the Value of Euler's Constant; likewise on the Values of the Napierian Logarithms of 2,3,5,7 and 10, and of the Modulus of common Logarithms, all carried to 260 places of Decimals, Proc. Roy. Soc. London, 27 (1878) 88-94. [3.15]

[3] L. Alaoglu and P. Erdős, On highly composite and similar numbers, Trans. Amer. Math. Soc. 56 (1944), 448-469. MR0011087 (6,117c) [3.4, 3.7]

[4] Y. André, G-Functions and Geometry, Friedr. Viehweg \& Sohn: Braunschweig 1989. [3.15]

[5] Yves André, G-fonctions et transcendance, J. Reine Angew. Math. 476 (1996), 95-125, DOI 10.1515/crll.1996.476.95 (French). MR 1401697 (98a:11097) [3.16] 
[6] Yves André, Séries Gevrey de type arithmétique. I. Théorèmes de pureté et de dualité, Ann. of Math. (2) 151 (2000), no. 2, 705-740, DOI 10.2307/121045 (French). MR.1765707 (2001m:11117) [3.15, 3.16]

[7] Yves André, Séries Gevrey de type arithmétique. II. Transcendance sans transcendance, Ann. of Math. (2) 151 (2000), no. 2, 741-756, DOI 10.2307/121046 (French). MR.1765708 (2001m:11118) [3.16]

[8] Yves André, Arithmetic Gevrey series and transcendence. A survey, J. Théor. Nombres Bordeaux 15 (2003), no. 1, 1-10 (English, with English and French summaries). Les XXIIèmes Journées Arithmetiques (Lille, 2001). MR2018997 (2004m:11115)] [3.16]

[9] Yves André, Une introduction aux motifs (motifs purs, motifs mixtes, périodes), Panoramas et Synthèses [Panoramas and Syntheses], vol. 17, Société Mathématique de France, Paris, 2004 (French, with English and French summaries). MR2115000 (2005k:14041) [3.14]

[10] R. Apéry, Irrationalité de $\zeta 2$ et $\zeta 3$, Astérisque No. 61 (1979), 11-13. [3.14, 3.15]

[11] R. Apéry, Interpolation de fractions continues et irrationalité de certaines constantes., Mathematics, pp. 37-53, CTHS: Bull. Sec. Sci., III, Bib. Nat., Paris 1981. [3.14, 3.15]

[12] A. I. Aptekarev (Editor), Rational approximants for Euler's constant and recurrence relations, Collected papers, Sovrem. Probl. Mat. ("Current Problems in Mathematics") Vol. 9, MIAN (Steklov Institute), Moscow (2007), 84pp (Russian) [3.16]

[13] A. I. Aptekarev, On linear forms containing the Euler constant, preprint arXiv.0902.1768. $[1.0,3.15]$

[14] A. I. Aptekarev, A. Branquinho, and W. Van Assche, Multiple orthogonal polynomials for classical weights, Trans. Amer. Math. Soc. 355 (2003), no. 10, 3887-3914, DOI 10.1090/S0002-9947-03-03330-0. MR1990569 (2004g:33014)] [3.15]

[15] A. I. Aptekarev and D. N. Tulyakov, On the determinant of an integral lattice generated by rational approximants of the Euler constant, Tr. Mosk. Mat. Obs. 70 (2009), 329-345 (Russian, with Russian summary); English transl., Trans. Moscow Math. Soc. (2009), 237249. MR2573642 (2010k:11107) [3.15]

[16] Richard Arratia, A. D. Barbour, and Simon Tavaré, On random polynomials over finite fields, Math. Proc. Cambridge Philos. Soc. 114 (1993), no. 2, 347-368, DOI 10.1017/S0305004100071620. MR1230136 (95a:60011) [3.11]

[17] Richard Arratia, A. D. Barbour, and Simon Tavaré, Random combinatorial structures and prime factorizations, Notices Amer. Math. Soc. 44 (1997), no. 8, 903-910. MR.1467654 (98i:60007) [3.11, 4.0]

[18] Richard Arratia, A. D. Barbour, and Simon Tavaré, The Poisson-Dirichlet distribution and the scale-invariant Poisson process, Combin. Probab. Comput. 8 (1999), no. 5, 407-416, DOI 10.1017/S0963548399003910. MR 1731976 (2001b:60023)] [3.11]

[19] Richard Arratia, A. D. Barbour, and Simon Tavaré, Logarithmic combinatorial structures: a probabilistic approach, EMS Monographs in Mathematics, European Mathematical Society (EMS), Zürich, 2003. MR2032426 (2004m:60004) [3.11]

[20] Emil Artin, The gamma function, Translated by Michael Butler. Athena Series: Selected Topics in Mathematics, Holt, Rinehart and Winston, New York, 1964. MR.0165148 (29 \#2437) [3.14]

[21] Raymond Ayoub, Euler and the zeta function, Amer. Math. Monthly 81 (1974), 1067-1086. MR0360116 (50 \#12566) [2.0, 2.4]

[22] A. I. Badzyan, The Euler-Kronecker constant, Mat. Zametki 87 (2010), no. 1, 35-47, DOI 10.1134/S0001434610010050 (Russian, with Russian summary); English transl., Math. Notes 87 (2010), no. 1-2, 31-42. MR2730381 [4.1]

[23] B. Baillaud, H. Bourget (Eds.), Correspondance d'Hermite et de Stieltjes, Tomes I, II. (With a preface by E. Picard and an appendix titled: Lettres de Stieltjes à Mittag-Leffler sur la fonction $\zeta(s)$ de Riemann), Gauthier-Villars, Paris, 1905. [3.1, 3.2]

[24] Keith Ball and Tanguy Rivoal, Irrationalité d'une infinité de valeurs de la fonction zêta aux entiers impairs, Invent. Math. 146 (2001), no. 1, 193-207, DOI 10.1007/s002220100168 (French). MR.1859021 (2003a:11086) [3.14]

[25] Dror Bar-Natan, On the Vassiliev knot invariants, Topology 34 (1995), no. 2, 423-472, DOI 10.1016/0040-9383(95)93237-2. MR 1318886 (97d:57004) [3.14]

[26] E. J. Barbeau and P. J. Leah, Euler's 1760 paper on divergent series, Historia Math. 3 (1976), no. 2, 141-160 (English, with French summary). MR0504847 (58 \#21162a) [2.5] 
[27] E. J. Barbeau, Euler subdues a very obstreperous series, Amer. Math. Monthly 86 (1979), no. 5, 356-372, DOI 10.2307/2321095. MR528791 (80i:01007) [2.5]

[28] Prakash Belkale and Patrick Brosnan, Periods and Igusa local zeta functions, Int. Math. Res. Not. 49 (2003), 2655-2670, DOI 10.1155/S107379280313142X. MR.2012522 (2004h:11097) [3.14]

[29] Jacob Bernoulli, Positione arithmeticarum de seriebus infinitis, earumque summa finita, quas auctero praeside Jac. Benoulli pro. virili tueri conabitur F. Jun 7, 1689. Basileae. [Basel 1689] [2.2, 2.4]

[30] Jacob Bernoulli, Tractatus de seriebus, pp. 242-306 in: Ars Conjectandi, opus posthumum, Accedit Tractatus de seriebus infinitis, et Epistola Gallicè scripta De ludo pilae reticularis, Basilae Impensis Thurnisiorum, Fratrum, 1713 [Basel 1713] [2.2, 2.4]

[31] F. Beukers, A note on the irrationality of $\zeta(2)$ and $\zeta(3)$, Bull. London Math. Soc. 11 (1979), no. 3, 268-272, DOI 10.1112/blms/11.3.268. MR554391 (81j:10045) [3.14, 4.0]

[32] F. Beukers, Padé-approximations in number theory, Padé approximation and its applications, Amsterdam 1980 (Amsterdam, 1980), Lecture Notes in Math., vol. 888, Springer, Berlin, 1981, pp. 90-99. MR649087 (83c:10049) [3.15]

[33] F. Beukers, Irrationality proofs using modular forms, Astérisque 147-148 (1987), 271-283, 345. Journées arithmétiques de Besançon (Besançon, 1985). MR891433 (88m:11055)] [3.15]

[34] Frits Beukers, W. Dale Brownawell, and Gert Heckman, Siegel normality, Ann. of Math. (2) 127 (1988), no. 2, 279-308, DOI 10.2307/2007054. MR932298 (90e:11106)] [3.16]

[35] Spencer Bloch and Hélène Esnault, Gauß-Manin determinant connections and periods for irregular connections, Geom. Funct. Anal. Special Volume (2000), 1-31, DOI 10.1007/9783-0346-0422-2_1. GAFA 2000 (Tel Aviv, 1999). MR1826247 (2002j:14012) [3.14]

[36] Jan Bohman and Carl-Erik Fröberg, The Stieltjes function-definition and properties, Math. Comp. 51 (1988), no. 183, 281-289, DOI 10.2307/2008591. MR942155(89i:11095) [3.2]

[37] W. A. Beyer and M. S. Waterman, Error analysis of a computation of Euler's constant, Math. Comp. 28 (1974), 599-604. MR0341809 (49 \#6555) [3.15]

[38] E. Bombieri, On G-functions, Recent progress in analytic number theory, Vol. 2 (Durham, 1979), Academic Press, London, 1981, pp. 1-67. MR637359 (83c:10050) [3.15]

[39] Enrico Bombieri and Jeffrey C. Lagarias, Complements to Li's criterion for the Riemann hypothesis, J. Number Theory 77 (1999), no. 2, 274-287, DOI 10.1006/jnth.1999.2392. MR 1702145 (2000h:11092) [3.2, 4.0]

[40] J.-B. Bost and A. Connes, Hecke algebras, type III factors and phase transitions with spontaneous symmetry breaking in number theory, Selecta Math. (N.S.) 1 (1995), no. 3, 411-457, DOI 10.1007/BF01589495. MR.1366621 (96m:46112)] [3.13]

[41] Robert E. Bradley and C. Edward Sandifer, Eds., Leonard Euler: Life, Work and Legacy, Studies in the History and Philosophy of Mathematics, Vol. 5, Elsevier, Amsterdam, 2007. $[2.0]$

[42] Richard P. Brent, Computation of the regular continued fraction for Euler's constant, Math. Comp. 31 (1977), no. 139, 771-777. MR0436547 (55 \#9490) [3.15]

[43] R. P. Brent, "Ramanujan and Euler's constant", in Mathematics of Computation 19431993: a half-century of computational mathematics (Vancouver, BC, 1993), pp. 541-545. Proc. Symp. Applied Math., Vol. 48, AMS: Providence, RI 1994. [4.0]

[44] Richard P. Brent and Edwin M. McMillan, Some new algorithms for high-precision computation of Euler's constant, Math. Comp. 34 (1980), no. 149, 305-312, DOI 10.2307/2006237. MR.551307 (82g:10002) [3.15]

[45] C. A. Bretschneider, Theoriae logarithmi integralis lineamenta nova, J. Reine Angew. Math. 17 (1837), 257-285. [2.6]

[46] William E. Briggs, Some constants associated with the Riemann zeta-function, Michigan Math. J. 3 (1955), 117-121. MR0076858 (17,955c)] [3.2]

[47] W. E. Briggs, The irrationality of $\gamma$ or of sets of similar constants, Norske Vid. Selsk. Forh. (Trondheim) 34 (1961), 25-28. MR0139579 (25 \#3011) [3.8]

[48] D. Broadhurst, Dickman polylogarithms and their constants, preprint arXiv:1004.0519 v1. $[4.0]$

[49] N. G. De Bruijn, On the number of uncancelled elements in the sieve of Eratosthenes, Nederl. Akad. Wetensch., Proc. 53 (1950), 803-812 = Indagationes Math. 12, 247-256 (1950). MR0035785(12,11d) [3.6] 
[50] N. G. de Bruijn, The asymptotic behaviour of a function occurring in the theory of primes, J. Indian Math. Soc. (N.S.) 15 (1951), 25-32. MR0043838(13,326f) [3.5]

[51] N. G. de Bruijn, On the number of positive integers $\leq x$ and free of prime factors $>y$, Nederl. Acad. Wetensch. Proc. Ser. A. 54 (1951), 50-60. MR0046375 (13,724e)] [3.5, 3.10]

[52] A. A. Buchstab, Asymptotic estimates of a general number-theoretic function (Russian. German summary), Math. Sbornik 2 (1937), no. 6, 1239-1246. [3.6]

[53] A. A. Buchstab, New improvements in the method of the sieve of Eratosthenes (Russian. German summary), Mat. Sbornik 4 (1938), no. 2, 375-387. [3.6]

[54] Ronald S. Calinger, "Leonard Euler: life and thought," in Leonard Euler: Life, Work and Legacy (Robert E. Bradley and C. Edward Sandifer, Eds.), Studies in the History and Philosophy of Mathematics, Vol. 5, pp. 5-60, Elsevier, Amsterdam, 2007. [2.0]

[55] Pierre Cartier, Fonctions polylogarithmes, nombres polyzêtas et groupes pro-unipotents, Astérisque 282 (2002), Exp. No. 885, viii, 137-173 (French, with French summary). Séminaire Bourbaki, Vol. 2000/2001. MR1975178 (2004i:19005)] [3.14]

[56] Jean-Marie-François Chamayou, A probabilistic approach to a differential-difference equation arising in analytic number theory, Math. Comp. 27 (1973), 197-203. MR.0336952 (49 \#1725) [3.5]

[57] A. Y. Cheer and D. A. Goldston, A differential delay equation arising from the sieve of Eratosthenes, Math. Comp. 55 (1990), no. 191, 129-141, DOI 10.2307/2008795. MR.1023043 $(90 \mathrm{j}: 11091)$ [3.6]

[58] S. Chowla, Improvement of a theorem of Linnik and Walfisz, Proc. London Math. Soc. (2) 50 (1949), 423-429. MR0027302 (10,285d)] [3.9]

[59] Mark W. Coffey, New results on the Stieltjes constants: asymptotic and exact evaluation, J. Math. Anal. Appl. 317 (2006), no. 2, 603-612, DOI 10.1016/j.jmaa.2005.06.048. MR2209581 (2007g:11106) [3.2]

[60] Mark W. Coffey, Addison-type representations for the Stieltjes constants, J. Number Theory 130 (2010), no. 9, 2049-2064. MR2653214(2011f:11111)] [3.2]

[61] Henri Cohen, Généralisation d'une construction de R. Apéry, Bull. Soc. Math. France 109 (1981), no. 3, 269-281 (French). MR680278 (84a:10036) [3.15]

[62] Henri Cohen, Number Theory-Volume II: Analytic and Modern Tools, Graduate Texts in Mathematics Vol. 240: Springer-Verlag: New York 2007. [3.2, 4.0]

[63] Joel E. Cohen and Charles M. Newman, The stability of large random matrices and their products, Ann. Probab. 12 (1984), no. 2, 283-310. MR735839 (86a:60013) [3.13]

[64] A. Connes and M. Marcolli, Non-commutative geometry, quantum fields and motives, Colloquium Publication no. 55, Amer. Math. Soc., Providence, RI 2008. [3.13]

[65] P. Deligne, Valeurs de fonctions $L$ et périodes d'intégrales, With an appendix by N. Koblitz and A. Ogus. Automorphic forms, representations and $L$-functions (Proc. Sympos. Pure Math., Oregon State Univ., Corvallis, Ore., 1977), Part 2, Proc. Sympos. Pure Math., XXXIII, Amer. Math. Soc., Providence, R.I., 1979, pp. 313-346 (French). MR546622 (81d:12009) [3.14]

[66] Pierre Deligne and Alexander B. Goncharov, Groupes fondamentaux motiviques de Tate mixte, Ann. Sci. École Norm. Sup. (4) 38 (2005), no. 1, 1-56, DOI 10.1016/j.ansens.2004.11.001 (French, with English and French summaries). MR2136480 (2006b:11066) [3.14]

[67] Christopher Deninger, Evidence for a cohomological approach to analytic number theory, First European Congress of Mathematics, Vol. I (Paris, 1992), Progr. Math., vol. 119, Birkhäuser, Basel, 1994, pp. 491-510. MR1341834 (96m:11075) [3.13]

[68] Christopher Deninger, Lefschetz trace formulas and explicit formulas in analytic number theory, J. Reine Angew. Math. 441 (1993), 1-15, DOI 10.1515/crll.1993.441.1. MR.1228608 (95d:11110) [3.13]

[69] Christopher Deninger, Some analogies between number theory and dynamical systems on foliated spaces, Proceedings of the International Congress of Mathematicians, Vol. I (Berlin, 1998), 1998, pp. 163-186 (electronic). MR.1648030 (99g:11084) [3.13]

[70] Christopher Deninger, On dynamical systems and their possible significance for arithmetic geometry, Regulators in Analysis, Geometry and Number Theory, Progr. Math., vol. 171, Birkhäuser Boston, Boston, MA, 2000, pp. 29-87. MR1724887 (2001g:11102) [3.13] 
[71] Harold G. Diamond and Kevin Ford, Generalized Euler constants, Math. Proc. Cambridge Philos. Soc. 145 (2008), no. 1, 27-41, DOI 10.1017/S0305004108001187. MR2431637 (2009g:11125) [3.8, 3.16]

[72] Jack Diamond, The p-adic log gamma function and p-adic Euler constants, Trans. Amer. Math. Soc. 233 (1977), 321-337. MR0498503 (58 \#16610) [4.0]

[73] K. Dickman, On the frequency of numbers containing prime factors of a certain relative magnitude, Arkiv för Mathematik, Astronomi och Fysik 22A (1930), 1-14. [3.5]

[74] Karl Dilcher, Generalized Euler constants for arithmetical progressions, Math. Comp. 59 (1992), no. 199, 259-282, S21-S24, DOI 10.2307/2152996. MR.1134726 (92k:11145) [3.8]

[75] G. L. Dirichlet Über die bestimmung der mittleren werthe in der Zahlentheorie, Abh. Akademie Berlin (1849), 69-83. [p. L. Dirichlet, Werke, Vol. II, 49-66. [3.4]

[76] V. G. Drinfel'd, Quasi-Hopf algebras, Algebra i Analiz 1 (1989), no. 6, 114-148 (Russian); English transl., Leningrad Math. J. 1 (1990), no. 6, 1419-1457. MR1047964 (91b:17016) $[3.14]$

[77] V. G. Drinfel'd, On quasitriangular quasi-Hopf algebras and on a group that is closely connected with $\operatorname{Gal}(\overline{\mathbf{Q}} / \mathbf{Q})$, Algebra i Analiz 2 (1990), no. 4, 149-181 (Russian); English transl., Leningrad Math. J. 2 (1991), no. 4, 829-860. MR.1080203 (92f:16047)] [3.14]

[78] V. G. Drinfel'd, On the structure of quasitriangular quasi-Hopf algebras, Funktsional. Anal. i Prilozhen. 26 (1992), no. 1, 78-80, DOI 10.1007/BF01077082 (Russian); English transl., Funct. Anal. Appl. 26 (1992), no. 1, 63-65. MR.1163026 (93i:16052)] [3.14]

[79] Jacques Dutka, The early history of the factorial function, Arch. Hist. Exact Sci. 43 (1991), no. 3, 225-249, DOI 10.1007/BF00389433. MR1171521 (93g:01034) [2.2, 2.3, 3.1]

[80] Bernard Dwork, Giovanni Gerotto, and Francis J. Sullivan, An introduction to G-functions, Annals of Mathematics Studies, vol. 133, Princeton University Press, Princeton, NJ, 1994. MR.1274045 (96c:12009) [3.15]

[81] H. M. Edwards, Riemann's Zeta Function, Academic Press: New York 1974. [2.3, 3.2]

[82] Emilio Elizalde, Ten physical applications of spectral zeta functions, Lecture Notes in Physics. New Series: Monographs, vol. 35, Springer-Verlag, Berlin, 1995. MR.1448403 (99b:58236) [3.2]

[83] E. Elizalde, S. D. Odintsov, A. Romeo, A. A. Bytsenko, and S. Zerbini, Zeta regularization techniques with applications, World Scientific Publishing Co. Inc., River Edge, NJ, 1994. MR:1346490 (96m:81156) [3.2]

[84] Emilio Elizalde, Luciano Vanzo, and Sergio Zerbini, Zeta-function regularization, the multiplicative anomaly and the Wodzicki residue, Comm. Math. Phys. 194 (1998), no. 3, 613-630, DOI 10.1007/s002200050371. MR.1631485(99e:58202) [3.2]

[85] C. Elsner, On a sequence transformation with integral coefficients for Euler's constant, Proc. Amer. Math. Soc. 123 (1995), no. 5, 1537-1541, DOI 10.2307/2161145. MR.1233969 (95f:11111) [4.0]

[86] G. Eneström, Verzeichnis der Schriften Leonhard Eulers, Jahresbericht der Deutschen Mathematiker-Vereinigung, Band IV, Part I, pp. 1-208 , B. G. Teubner: Leipzig 1910; Part II, pp. 209-398, B. G. Teubner: Leipzig 1913. [English Translation by Greta Perl: available on the Euler Archive, also available online at http://www.math.dartmouth.edu/ euler/docs/ translations/enestrom/Enestrom-index.pdf] [2.0]

[87] H. Erdélyi, W. Magnus, F. Oberhettinger, and F. G. Tricomi, Higher Transcendental Functions, Vol. I, McGraw-Hill, New York 1953. [3.1, 3.15]

[88] The Euler Archive: The works of Leonhard Euler online, http://www.eulerarchive.org $[2.0]$

[89] L. Euler, De progressionibus transcendentibus seu quarum termini generales algebraice dari nequent, Comment. Acad. Sci. Petrop. 5 (1738), 36-57. (Presented Nov. 8, 1729) (Opera Omnia, Series 1, Vol. 14, pp. 1-24) [E19] [2.3]

[90] L. Euler, De summatione innumerabilium progressionum, Comment. Acad. Sci. Petrop. 5 (1738), 91-105. (presented March 5, 1731) (Opera Omnia, Series 1, Vol. 14, pp. 25-41). [E20] $[2.2,2.4]$

[91] L. Euler, Methodus generalis summandi progressiones, Comment. Acad. Sci. Petrop. 6 (1738), 68-97. (Presented June 20, 1732) (Opera Omnia, Series 1, Vol. 14, pp. 42-72) [E25] $[2.2,2.4]$

[92] L. Euler, De summis serierum reciprocarum, Comment. Acad. Sci. Petrop. 7 (1740), 123134. (Read Dec. 5, 1735). (Opera Omnia, Series 1, Vol. 14, pp. 73-86.) [E41] [2.4] 
[93] L. Euler, De progressionibus harmonicis observationes, Comment. Acad. Sci. Petrop. 7 (1740), 150-161. (Read March 11, 1734, not turned in). (Opera Omnia, Series 1, Vol. 14, pp. 87-100.) [E43] [2.2]

[94] L. Euler, Inventio summae cuiusque seriei ex dato termino generali, Comment. Acad. Sci. Petrop. 8 (1741), 9-22 (Presented Oct. 13, 1735). (Opera Omnia, Series I, Vol. 14, pp. 108123). [E47] [2.2, 2.4]

[95] L. Euler, De summis serierum reciprocarum ex potestatibus numerorum naturalium ortarum Dissertatio altera, in qua eadem summationes ex fonte maxime diverso derivantur, (Presented Sept. 6, 1742), Miscellanea Berolinensia 7 (1743), 172-192. (Opera Omnia, Series 1, Vol. 14, 138-155.) [E61] [2.4]

[96] L. Euler, Demonstration de la somme de cette suite $1+1 / 4+1 / 9+1 / 16 \ldots$, Journal Lit. d'Allemagne, de Suisse et du Nord, 2, no. 1 (1743), 115-127. (Opera Omnia, Series 1, Vol. 14, pp. 177-186.) [E63] [2.4]

[97] L. Euler, De fractionibus continuis dissertatio, Comment. Acad. Sci. Petrop. 9 (1744), 98137. (Opera Omnia, Series 1, Vol. 14, 187-216) [E71] [1.1, 2.3]

[98] Leonhard Euler, An essay on continued fractions, Translated from the Latin by B. F. Wyman and M. F. Wyman, Math. Systems Theory 18 (1985), no. 4, 295-328, DOI 10.1007/BF01699475. MR818419(87d:01011b) [1.1]

[99] L. Euler, Variae observations circa series infinitas, Comment. Acad. Sci. Petrop. 9 (1744), 160-188. (Presented April 25, 1737). (Opera Omnia, Series 1, Vol. 14, pp. 217-244.) [E72] $[2.4]$

[100] L. Euler, De fractionibus continuis observationes, Comm. Acad. Sci. Petrop. 11 (1750), pp. 32-81. (Presented January 22, 1739). (Opera Omnia, Series 1, Vol. 14, pp. 291-349.) [English translation in: Appendix to [Khr08], pp. 426-465.] [E123] [2.5]

[101] L. Euler, De seriebus quibusdam considerationes, Comment. Acad. Sci. Petrop. 12 (1750), 53-96. [Presented Oct. 22, 1739) (Opera Omnia, Series 1, Vol. 14, pp. 407-462.) [E130] [2.4]

[102] L. Euler, Calcul de la Probabilité dans le jeu de recontre, Mémoires de l'Académie des Sciences de Berlin 7 (1753), 255-270. (Opera Omni, Series 1, Volume 7, pp. 11-25.) [E201] [3.11]

[103] L. Euler, Institutiones calculi differentialis cum eius usu in analysi finitorum ac doctrina serierum, Imprinti Academiae Imperialis Scientarum Petropolitanae 1755. (Opera Omnia, Series 1, Volume 10.) [E212] [2.2, 2.4]

[104] L. Euler, De seriebus divergentibus, Novi. Commentii Acad. Sci. Petrop. 5 (1760), 205-237. (Opera Omnia, Series 1, Vol. 14, pp. 585-617.) [E247] [2.5]

[105] L. Euler, Remarques sur un beau rapport entre les séries des puissances tant directes qui réciproques, Mémoires de l'Académie des Sciences de Berlin 17 (1768), 83-106. (Presented (Opera Omnia, Series 1, Vol. 15, pp. 70-90.) [E352] [2.4]

[106] L. Euler, De curva hypegeometrica hac aequatione expressa $y=1.2 .3 \ldots \ldots x$, Novi Coment. Acad. Sci. Petrop. 13 (1769), 3-66. (Presented Dec. 19, 1765) (Opera Omnia, Series 1, Vol. 28, pp. 41-98.) [E368]. [2.2, 2.3, 2.4, 3.1]

[107] L. Euler, De summis serierum numeros Bernoullianos involventinum, Novi Comment. Acad. Sci. Petrop. 14 (1770), 129-167. (Presented Aug. 18, 1768) (Opera Omnia, Series I, Vol. 15, pp. 91-130) [E393] [2.2]

[108] L. Euler, Exercitationes Analyticae, Novi. Comment. Acad. Sci. Petrop. 17 (1773), 173-204. (Presented May 18, 1772)(Opera Omnia, Series 1, Vol. 15, 131-167.) [E432] [2.4]

[109] L. Euler, Meditationes circa singulare serierum genus, Novi. Comment. Acad. Sci. Petrop. 20 (1776), 140-186. (Presented July 4, 1771). (Opera Omnia, Series 1, Vol. 15, pp. 217-267.) [E477] [2.4]

[110] L. Euler, De numero memorabili in summatione progressionis harmonicae naturalis occurrente, Acta Acad. Scient. Imp. Petrop. 5 (1785), 45-75. (Presented Feb. 22, 1776). (Opera Omnia, Ser. 1, Vol. 15, pp. 569-603) [E583] [2.2]

[111] L. Euler, De seriebus potestatum reciprocis methodo nova et facillima summandis, Opuscula Analytica 2 (1785), 257-274. (Presented Oct. 2, 1775) (Opera Omnia, Ser. 1, Vol. 15, pp. 701-720) [E597] [2.4]

[112] L. Euler, Evolutio formulae integralis $\int d x\left(\frac{1}{1-x}+\frac{1}{l x}\right)$ a termino $x=0$ usque ad $x=1$ extensae, Nova Acta Acad. Scient. Imp. Petrop. 4 (1789), 3-16 (Presented Feb. 29, 1776). (Opera Omnia, Series I, Vol. 18, pp. 318-334) [E629] [2.2, 2.6] 
[113] L. Euler, De summatione serierum in hac forma contentarum $\frac{a}{1}+\frac{a^{2}}{4}+\frac{a^{3}}{9}+\frac{a^{4}}{16}+\frac{a^{5}}{25}+\frac{a^{6}}{36}+$ etc., Memoires de l'Academie des Sciences de St.-Petersbourg 3 (1811), 26-42. (Presented May 29, 1779) (Opera Omnia, Series 1, Vol. 16, pp. 117-138) [E736] [2.4]

[114] N. I. Feldman and Yu. V. Nesterenko, Transcendental Numbers. Translation of Number Theory 4. (Edited by A. N. Parshin and I. R. Shafarevich) Encyclopedia of Mathematical Sciences 44, Springer-Verlag: berlin 1998. [3.15]

[115] Giovanni Ferraro, The foundational aspects of Gauss's work on the hypergeometric, factorial and digamma functions, Arch. Hist. Exact Sci. 61 (2007), no. 5, 457-518, DOI 10.1007/s00407-007-0004-8. MR2329096 (2009d:33002)] [3.1]

[116] Steven R. Finch, Mathematical constants, Encyclopedia of Mathematics and its Applications, vol. 94, Cambridge University Press, Cambridge, 2003. MR 2003519 (2004i:00001) $[1.0,2.5,3.10,3.13]$

[117] Stéphane Fischler, Irrationalité de valeurs de zêta (d'après Apéry, Rivoal, ...), Astérisque 294 (2004), vii, 27-62 (French, with French summary). MR2111638(2005i:11091)] [3.14]

[118] S. Fischler and T. Rivoal, On the values of G-functions, Comm. Math. Helv., to appear.

[119] Philippe Flajolet and Andrew M. Odlyzko, Random mapping statistics, Advances in cryptology_EUROCRYPT '89 (Houthalen, 1989), Lecture Notes in Comput. Sci., vol. 434, Springer, Berlin, 1990, pp. 329-354, DOI 10.1007/3-540-46885-4_34. MR_1083961] [3.12]

[120] Philippe Flajolet and Andrew Odlyzko, Singularity analysis of generating functions, SIAM J. Discrete Math. 3 (1990), no. 2, 216-240, DOI 10.1137/0403019. MR.1039294 (90m:05012) [3.11]

[121] John Friedlander and Henryk Iwaniec, Opera de cribro, American Mathematical Society Colloquium Publications, vol. 57, American Mathematical Society, Providence, RI, 2010. MR 2647984 (2011d:11227) [3.6]

[122] H. Furstenberg and H. Kesten, Products of random matrices, Ann. Math. Statist. 31 (1960), 457-469. MR0121828 (22 \#12558)] [3.13]

[123] A. Galochkin, Estimates from below of polynomials in the values of analytic functions in a certain class, Math. USSR Sbornik 24 (1974), 385-407. (Russian original: Mat. Sbornik 95 (137) (1974), 396-417, 471.) [3.15]

[124] C. F. Gauss, Disquisitiones Generales circa seriem infinitam $1+\frac{\alpha \beta x}{1 \cdot \gamma}+\frac{\alpha(\alpha+1) \beta(\beta+1) x x}{1 \cdot 2 \gamma(\gamma+1)}+\cdots$, Commentationes Gottingensis 2 (1813). [Also pp. 123-162 in; Werke, Band III, Kóniglichen Geschellshaft der Wissenschaften, Gótiinger 1866.] [3.1]

[125] Walter Gautschi, Leonhard Euler: his life, the man, and his works, SIAM Rev. 50 (2008), no. 1, 3-33, DOI 10.1137/070702710. MR2403056 (2009d:01016) [2.0]

[126] E. Giusti, Le prime richerche di Pietro Mengoli. La somma della serie. In: Coen, S. Ed, Geometry and Complex Variables: Proceedings of an International Meeting on the Occasion of the IX Centennial of the University of Bologna, Marshall Decker: New York 1991, pp. 195213. [2.4]

[127] J. W. L. Glaisher, On the calculation of Euler's constant, Proc. Roy. Soc. London 19 (1871), 510-522. [3.15]

[128] J. W. L. Glaisher, On the history of Euler's constant, Messenger of Mathematics 1 (1872), 25-30. [3.15]

[129] S. W. Golomb, L. R. Welch and R. M. Goldstein, Cycles from nonlinear shift registers, Progress Report no. 20-389, Jet Propulsion Laboratory, California Institute of Technology, Pasadena, CA 1959. [3.10]

[130] S. W. Golomb, Research Problem 11: Random Permutations, Bull. Amer. Math. Soc. 70 (1964), no. 6, 747. [3.10]

[131] Solomon W. Golomb, Shift register sequences, With portions co-authored by Lloyd R. Welch, Richard M. Goldstein, and Alfred W. Hales, Holden-Day Inc., San Francisco, Calif., 1967. MR0242575 (39 \#3906) [3.10]

[132] Benjamin Gompertz, On the nature of the function expressive of the law of human mortality, and on a new mode of determining the value of life contingencies, Phil. Trans. Roy. Soc. London 115 (1825), 513-585. [2.5]

[133] Benjamin Gompertz, A supplement to Two Papers Published in the Transactions of the Royal Society, "On the Science Connected with Human Mortality;" the One Published in 1820 and the Other in 1825, Phil. Trans. Roy. Soc. London, 152 (1862), 511-559. [2.5]

[134] W. Gontcharoff, Sur la distribution des cycles dans les permutations, C. R. (Doklady) Acad. Sci. URSS (N.S.) 35 (1942), 267-269. MR0007207 (4,102g) [3.10] 
[135] V. Goncharov, Du domaine d'analyse combinatoire, Izv. Akad. Nauk SSSR 8 (1944), 3-48. [English Translation: Amer. Math. Soc. Transl. (2), Vol. 19 (1962), 1-46. [3.10]

[136] R. Wm. Gosper Jr., A calculus of series rearrangements, Algorithms and complexity (Proc. Sympos., Carnegie-Mellon Univ., Pittsburgh, Pa., 1976), Academic Press, New York, 1976, pp. 121-151. MR0451617 (56 \#9899)] [2.5, 2.15]

[137] R. W. Gosper, Strip mining in the abandoned ore fields of nineteenth century mathematics, in: Computers in Mathematics (Stanford, CA 1986), pp. 261-284, Lecture Notes in Pure and Appied Math., 125, Dekker, New York 1990. [3.15]

[138] X. Gourdon, Combinatoire, Algorithmiques et Géométrie des Polynômes, Ph. D. Thesis, École Polytechnique 1996. [3.10]

[139] Andrew Granville, Unexpected irregularities in the distribution of prime numbers, 2 (Zürich, 1994), Birkhäuser, Basel, 1995, pp. 388-399. MR.1403939 (97d:11139) [3.3]

[140] Andrew Granville, Smooth numbers: computational number theory and beyond, Algorithmic number theory: lattices, number fields, curves and cryptography, Math. Sci. Res. Inst. Publ., vol. 44, Cambridge Univ. Press, Cambridge, 2008, pp. 267-323. MR2467549 (2010g:11214) [3.5]

[141] A. Granville, The anatomy of integers and permutations, preprint. [Ack]

[142] A. Granville and K. Soundararajan, The distribution of values of $L\left(1, \chi_{d}\right)$, Geom. Funct. Anal. 13 (2003), no. 5, 992-1028, DOI 10.1007/s00039-003-0438-3. MR2024414 (2005d:11129) [3.9]

[143] Andrew Granville and K. Soundararajan, Extreme values of $|\zeta(1+i t)|$, The Riemann zeta function and related themes: papers in honour of Professor K. Ramachandra, Ramanujan Math. Soc. Lect. Notes Ser., vol. 2, Ramanujan Math. Soc., Mysore, 2006, pp. 65-80. MR2335187 (2008f:11091) [3.9]

[144] R. E. Greenwood, The number of cycles associated with the elements of a permutation group, Amer. Math. Monthly 60 (1953), 407-409. MR0054552 (14,939b) [3.10]

[145] T. H. Gronwall, Some asymptotic expressions in the theory of numbers, Trans. Amer. Math. Soc. 14 (1913), no. 1, 113-122, DOI 10.2307/1988773. MR 1500940 [3.4]

[146] Benedict H. Gross, On the periods of abelian integrals and a formula of Chowla and Selberg, Invent. Math. 45 (1978), no. 2, 193-211. With an appendix by David E. Rohrlich. MR:0480542 (58 \#701) [3.14]

[147] Osias Gruder, Zur Theorie der Zerlegung von Permutationen in Zyklen, Ark. Mat. 2 (1952), 385-414 (German). MR0051202 (14,442a) [3.11]

[148] Jesús Guillera and Jonathan Sondow, Double integrals and infinite products for some classical constants via analytic continuations of Lerch's transcendent, Ramanujan J. 16 (2008), no. 3, 247-270, DOI 10.1007/s11139-007-9102-0. MR2429900 (2009e:11239)] [4.0]

[149] Li Guo, Sylvie Paycha, Bingyong Xie, and Bin Zhang, Double shuffle relations and renormalization of multiple zeta values, The geometry of algebraic cycles, Clay Math. Proc., vol. 9, Amer. Math. Soc., Providence, RI, 2010, pp. 145-187. MR2648671 (2011h:11094) [4.0]

[150] Li Guo and Bin Zhang, Renormalization of multiple zeta values, J. Algebra 319 (2008), no. 9, 3770-3809, DOI 10.1016/j.jalgebra.2008.02.003. MR2407850(2009b:11155) [4.0]

[151] Li Guo and Bin Zhang, Differential algebraic Birkhoff decomposition and the renormalization of multiple zeta values, J. Number Theory 128 (2008), no. 8, 2318-2339, DOI 10.1016/j.jnt.2007.10.005. MR.2394823 (2009i:11109) [4.0]

[152] L. A. Gutnik, Irrationality of some quantities that contain $\zeta(3)$, Acta Arith. 42 (1983), no. 3, 255-264 (Russian). MR729735 (85j:11079) [3.15]

[153] M. J. Shai Haran, The mysteries of the real prime, London Mathematical Society Monographs. New Series, vol. 25, The Clarendon Press, Oxford University Press, New York, 2001. MR:1872029 (2003b:11085) [4.0]

[154] G. H. Hardy, Note on Dr. Vacca's series for $\gamma$, Quart. J. Pure. Appl. Math. (Oxford) 43 (1912), 215-216. [3.2]

[155] G. H. Hardy, On Dirichlet's Divisor Problem, Proc. London Math. Soc., Series 2, 15 (1916), no. 1, 1-25, DOI 10.1112/plms/s2-15.1.1. MR1576550 [3.4]

[156] G. H. Hardy, Divergent Series, Oxford, at the Clarendon Press, 1949. MR0030620 (11,25a) $[2.4,2.5]$

[157] G. H. Hardy and E. M. Wright, An introduction to the theory of numbers, With a foreword by Andrew Wiles, 6th ed., Oxford University Press, Oxford, 2008. Revised by D. R. HeathBrown and J. H. Silverman. MR2445243(2009i:11001)] [3.3] 
[158] Julian Havil, Gamma, Exploring Euler's constant; With a foreword by Freeman Dyson, Princeton University Press, Princeton, NJ, 2003. MR1968276 (2004k:11195)] [1.0, 2.6]

[159] S. W. Hawking, Zeta function regularization of path integrals in curved spacetime, Comm. Math. Phys. 55 (1977), no. 2, 133-148. MR0524257 (58 \#25823)] [3.2]

[160] G. Herglotz, Über die Kornecksche Grenzformel für reelle quadratische Körper I, II, Berichte über die Verhandl. d. Sächsischen Akad. der Wiss. zu Leipzig 75 (1923), 3-14, 31-37. [4.0]

[161] Khodabakhsh Hessami Pilehrood and Tatiana Hessami Pilehrood, Arithmetical properties of some series with logarithmic coefficients, Math. Z. 255 (2007), no. 1, 117-131, DOI 10.1007/s00209-006-0015-1. MR2262724(2008b:11081)] [4.0]

[162] Kh. Hessami Pilehrood and T. Hessami Pilehrood, Rational approximations for the quotient of gamma values, Indag. Math. (N.S.) 20 (2009), no. 4, 583-601, DOI 10.1016/S00193577(09)80027-X. MR2776901 (2012i:33005) [3.15]

[163] Kh. Hessami Pilehrood and T. Hessami Pilehrood, On a continued fraction expansion for Euler's constant, J. Number Theory 133 (2013), no. 2, 769-786, DOI 10.1016/j.jnt.2012.08.016. MR2994386 [3.15]

[164] Adolf Hildebrand, The asymptotic behavior of the solutions of a class of differentialdifference equations, J. London Math. Soc. (2) 42 (1990), no. 1, 11-31, DOI 10.1112/jlms/s242.1.11. MR:1078172 (92f:11123)] [3.6]

[165] Adolf Hildebrand and Gérald Tenenbaum, Integers without large prime factors, J. Théor. Nombres Bordeaux 5 (1993), no. 2, 411-484. MR.1265913 (95d:11116) [3.5]

[166] F. B. Hildebrand, Introduction to numerical analysis, McGraw-Hill Book Company, Inc., New York-Toronto-London, 1956. MR0075670 (17,788d) [2.4]

[167] Jos. E. Hofmann, Zur Entwicklungsgeschichte der Eulerschen Summenformel, Math. Z. 67 (1957), 139-146 (German). MR0085983 (19,108a) [2.0]

[168] M. N. Huxley, Exponential sums and lattice points. III, Proc. London Math. Soc. (3) 87 (2003), no. 3, 591-609, DOI 10.1112/S0024611503014485. MR2005876 (2004m:11127) [3.4]

[169] M. N. Huxley, Exponential sums and the Riemann zeta function. $V$, Proc. London Math. Soc. (3) 90 (2005), no. 1, 1-41, DOI 10.1112/S0024611504014959. MR2107036 (2005h:11180) [3.4]

[170] Ts. Ignatov, On a constant arising in the asymptotic theory of symmetric groups, and on Poisson-Dirichlet measures, Theor. Prob. Appl. 27 (1982), no. 1, 136-147. MR0645134 (83i:10072) [3.11]

[171] Kentaro Ihara, Masanobu Kaneko, and Don Zagier, Derivation and double shuffle relations for multiple zeta values, Compos. Math. 142 (2006), no. 2, 307-338, DOI 10.1112/S0010437X0500182X. MR2218898(2007e:11110) [4.0]

[172] Yasutaka Ihara, On the Euler-Kronecker constants of global fields and primes with small norms, Algebraic geometry and number theory, Progr. Math., vol. 253, Birkhäuser Boston, Boston, MA, 2006, pp. 407-451, DOI 10.1007/978-0-8176-4532-8_5. MR.2263195 (2007h:11127) [4.0]

[173] J. B. Keiper, Power series expansions of Riemann's $\xi$ function, Math. Comp. 58 (1992), no. 198, 765-773, DOI 10.2307/2153215. MR 1122072 (92f:11116) [3.2]

[174] A. Ya. Khinchin, Continued Fractions, Third Edition. (English Transl. p. Wynn), Nordhoff: Groningen 1963. [3.15]

[175] Sergey Khrushchev, Orthogonal polynomials and continued fractions, From Euler's point of view. Encyclopedia of Mathematics and its Applications, vol. 122, Cambridge University Press, Cambridge, 2008. MR2442472 (2010m:42001) [2.5]

[176] Charles Knessl and Mark W. Coffey, An effective asymptotic formula for the Stieltjes constants, Math. Comp. 80 (2011), no. 273, 379-386, DOI 10.1090/S0025-5718-2010-02390-7. MR2728984 (2011k:11180) [3.2]

[177] Charles Knessl and Mark W. Coffey, An asymptotic form for the Stieltjes constants $\gamma_{k}(a)$ and for a sum $S_{\gamma}(n)$ appearing under the Li criterion, Math. Comp. 80 (2011), no. 276, 2197-2217. MR2728984(2011k:11180) [3.2]

[178] Arthur Knoebel, Reinhard Laubenbacher, Jerry Lodder, and David Pengelley, Mathematical masterpieces, Further chronicles by the explorers; Undergraduate Texts in Mathematics, Springer, New York, 2007. MR2337639 [2.0]

[179] Donald E. Knuth, Euler's constant to 1271 places, Math. Comp. 16 (1962), 275-281. MR.0148255(26 \#5763)[3.15] 
[180] Donald E. Knuth and Luis Trabb-Pardo, Analysis of a simple factorization algorithm, Theoret. Comput. Sci. 3 (1976/77), no. 3, 321-348. MR0498355 (58 \#16485) [1.0, 3.5, 3.10, $3.11,3.12]$

[181] Neal Koblitz, Interpretation of the p-adic log gamma function and Euler constants using the Bernoulli measure, Trans. Amer. Math. Soc. 242 (1978), 261-269. MR0491622(58 \#10836) $[4.0]$

[182] Valentin F. Kolchin, Random mappings, With a foreword by S. R. S. Varadhan. Translation Series in Mathematics and Engineering, Optimization Software Inc. Publications Division, New York, 1986. Translated from the Russian. MR865130(88a:60022) [3.12]

[183] Margo Kondratieva and Sergey Sadov, Markov's transformation of series and the WZ method, Adv. in Appl. Math. 34 (2005), no. 2, 393-407, DOI 10.1016/j.aam.2004.06.003. MR2110559 (2005k:65006) [3.15]

[184] Maxim Kontsevich, Vassiliev's knot invariants, I. M. Gel'fand Seminar, Adv. Soviet Math., vol. 16, Amer. Math. Soc., Providence, RI, 1993, pp. 137-150. MR.1237836 (94k:57014) $[3.14]$

[185] Maxim Kontsevich, Operads and motives in deformation quantization, Moshé Flato (1937-1998), Lett. Math. Phys. 48 (1999), no. 1, 35-72, DOI 10.1023/A:1007555725247. MR 1718044 (2000j:53119) [3.14]

[186] Maxim Kontsevich and Don Zagier, Periods, Mathematics unlimited - 2001 and beyond, Springer, Berlin, 2001, pp. 771-808. MR1852188(2002i:11002) [1.0, 3.14]

[187] L. Kronecker, Zur Theorie der ellptische Modulfunktionen, Werke, Vol. 4, 347-395 and Vol. $5,1-132 .[4.0]$

[188] Nobushige Kurokawa and Masato Wakayama, On q-analogues of the Euler constant and Lerch's limit formula, Proc. Amer. Math. Soc. 132 (2004), no. 4, 935-943 (electronic), DOI 10.1090/S0002-9939-03-07025-4. MR2045407 (2005d:33022) [4.0]

[189] Ch.-J. de La Vallee Poussin, Sur les valeurs moyennes de certaines fonctions arithmétiques, Annales de la Societe Scientifique de Bruxelles 22 (1898), 84-90 [3.4]; [in Collected Works Oeuvres Scientifiques, Vol I., ed. p. Butzer, J. Mawhin, P.Vetro. Acad. Royale Belgique, Circ. Math. Palermo, 2000, pp. 559-564]. [3.4]

[190] Jeffrey C. Lagarias, Number theory zeta functions and dynamical zeta functions, Spectral problems in geometry and arithmetic (Iowa City, IA, 1997), Contemp. Math., vol. 237, Amer. Math. Soc., Providence, RI, 1999, pp. 45-86, DOI 10.1090/conm/237/1710789. MR/1710789 (2001a:11153) [3.13]

[191] Jeffrey C. Lagarias, An elementary problem equivalent to the Riemann hypothesis, Amer. Math. Monthly 109 (2002), no. 6, 534-543, DOI 10.2307/2695443. MR1908008 (2003d:11129) [3.7]

[192] Jeffrey C. Lagarias, The Riemann hypothesis: arithmetic and geometry, Surveys in noncommutative geometry, Clay Math. Proc., vol. 6, Amer. Math. Soc., Providence, RI, 2006, pp. 127-141. MR2277670 (2008g:11140) [3.13]

[193] E. Laguerre, Sur l'integrale $\int_{x}^{\infty} \frac{e^{-x} d x}{x}$, Bull. Soc. Math. France, Series 1, 7 (1879), $72-81$. [Also: C. Hermite, H. Poincaré, E. Rouché, Eds., Oeuvres de Laguerre, Tome I, GautiersVilliars, Paris 1905, pp. 428-437.] [2.5]

[194] Youness Lamzouri, The two-dimensional distribution of values of $\zeta(1+i t)$, Int. Math. Res. Not. IMRN, posted on 2008, Art. ID rnn 106, 48, DOI 10.1093/imrn/rnn106. MR2439537 (2010c:11104) [3.9]

[195] E. Landau, Uber den Verlauf der zahlentheoretischen Funktion $\varphi(x)$, Archiv der Mathematik und Physik, Ser. 3. 5 (1903), 86-91. [3.4]

[196] E. Landau, Handbuch der Lehre von der Verteilung der Primzahlen, Teubner: Leipzig 1909 (Reprinted by Chelsea Publ. Co.). [3.4]

[197] Michel L. Lapidus, In search of the Riemann zeros, Strings, fractal membranes and noncommutative spacetimes. American Mathematical Society, Providence, RI, 2008. MR2375028 (2009f:11110) [3.13]

[198] Peter D. Lax and Ralph S. Phillips, Scattering theory for automorphic functions, Annals of Mathematics Studies, No. 87. Princeton Univ. Press, Princeton, N.J., 1976. MR0562288 (58 \#27768) [3.9]

[199] Peter D. Lax and Ralph S. Phillips, Scattering theory for automorphic functions, Bull. Amer. Math. Soc. (N.S.) 2 (1980), no. 2, 261-295, DOI 10.1090/S0273-0979-1980-14735-7. MR 555264(81c:10037)] [3.9] 
[200] D. H. Lehmer, On reciprocally weighted partitions, Acta Arith. 21 (1972), 379-388. MR.0304302(46 \#3437) [3.10]

[201] D. H. Lehmer, Euler constants for arithmetical progressions, Acta Arith. 27 (1975), 125142. Collection of articles in memory of Juriǔ Vladimirovič Linnik. MR0369233 (51 \#5468) $[3.8,3.16]$

[202] George Leibbrandt, Introduction to the technique of dimensional regularization, Rev. Modern Phys. 47 (1975), no. 4, 849-876. MR0438957 (55 \#11859) [3.2]

[203] Francois Le Lionnais, Les nombres remarquables, Hermann, Paris 1983 [2.5]

[204] J. H. van Lint and H. E. Richert, Über die summe $\sum_{\substack{n \leq x \\ p(n)<y}} \frac{\mu^{2}(n)}{\phi(n)}$, Koninkl. Nederl. Akad. Wetenshaft Proc. Ser. A, 67 (1964), no. 5, 582-587. (Indag. Math. 26 (1964), no. 5, 582-587.) MR0171767 (30 \#1994) [3.5]

[205] J. E. Littlewood, On the Riemann Zeta-Function, Proc. London Math. Soc. S2-24, no. 1, 175, DOI 10.1112/plms/s2-24.1.175. MR1577156 [3.9]

[206] J. E. Littlewood, Mathematical Notes (5): On the function $\frac{1}{\zeta(1+t i)}$, Proc. London Math. Soc. 27 (1928), 349-357. [3.9]

[207] J. E. Littlewood, On the Class-Number of the Corpus P(surd-k), Proc. London Math. Soc. S2-27, no. 1, 358, DOI 10.1112/plms/s2-27.1.358. MR.1575396 [3.9]

[208] C. Maclaurin, A Treatise of Fluxions: In two books. T. W. and T. Ruddimans, Edinburgh, 1742,763 pp. [2.2]

[209] K. Mahler, Applications of a theorem by A. B. Shidlovski, Proc. Roy. Soc. Ser. A 305 (1968), 149-173. MR0225729 (37 \#1322) [3.16]

[210] Helmut Maier, Primes in short intervals, Michigan Math. J. 32 (1985), no. 2, 221-225, DOI $10.1307 / \mathrm{mmj} / 1029003189 . \mathrm{MR} 783576(86 \mathrm{i}: 11049)[3.3,3.6]$

[211] Dominique Manchon and Sylvie Paycha, Nested sums of symbols and renormalized multiple zeta values, Int. Math. Res. Not. IMRN 24 (2010), 4628-4697, DOI 10.1093/imrn/rnq027. MR2739806 (2012a:81184) [4.0]

[212] A. A. Markoff, Mémoire sur la transformation des séries peu convergentes et séries trés convergentes, Mém. Acad. Imp. Sci. St. Pétersbourg, 37 (9) (1890), 18. [3.15]

[213] L. Mascheroni, Adnotationes ad calculum integralem Euleri, in quibus nonullae problemata ab Eulero proposita resolvuntur, Galeiti, Ticini 1790. [2.6]

[214] L. Mascheroni, Adnotationes ad calculum integralem Euleri, in quibus nonullae formulae ab Eulero propositae evolvuntur, Galeiti, Ticini 1792. [2.6]

[215] Ma. Rosa Massa Esteve, Algebra and geometry in Pietro Mengoli (1625-1686), Historia Math. 33 (2006), no. 1, 82-112, DOI 10.1016/j.hm.2004.12.003 (English, with English and French summaries). MR2204951 (2006i:01013) [2.4]

[216] C. S. Meijer, Über Wittakersche Bezw. Besslsche Funktionen und deren Produkte, Nieuw. Archief Wisk. (2) 18 (1936), 10-39. [3.15]

[217] P. Mengoli, Novae Quadraturae Arithmeticae, seu De Additione Fractionum ... Bologna 1650. [2.4]

[218] Nicholas Mercator, Logarithmo-technia, sive Methodus construendi Logarithmos, Nova, accurata \& facilis, scripto Antehàc Communicata, Anno Sc. 1667, Nonis Augusti: Cui nunc accedit. Vera Quadratura Hyperbolae 65 Inventio summae Logarithmorum. Londini 1668 [London 1668] [2.2]

[219] F. Mertens, Ein Beitrag zur analytischen Zahlentheorie, J. Reine Angew. Math. 78 (1874), 46-62. [3.3]

[220] Stella Mills, The independent derivations by Leonhard Euler and Colin Maclaurin of the Euler-Maclaurin summation formula, Arch. Hist. Exact Sci. 33 (1985), no. 1-3, 1-13, DOI 10.1007/BF00328047. MR.795457 (86i:01025) [2.2]

[221] W. C. Mitchell, An evaluation of Golomb's constant, Math. Comp. 22 (1968), 411-415. $[3.10,3.11]$

[222] H. L. Montgomery and R. C. Vaughan, Extreme values of Dirichlet L-functions at 1, Number theory in progress, Vol. 2 (Zakopane-Kościelisko, 1997), de Gruyter, Berlin, 1999, pp. 10391052. MR 1689558 (2000m:11075) [3.9]

[223] Hugh L. Montgomery and Robert C. Vaughan, Multiplicative number theory. I. Classical theory, Cambridge Studies in Advanced Mathematics, vol. 97, Cambridge University Press, Cambridge, 2007. MR2378655 (2009b:11001) [3.6] 
[224] Pieter Moree, Psixyology and Diophantine equations, Rijksuniversiteit te Leiden, Leiden, 1993 (English, with Dutch summary). Dissertation, Rijksuniversiteit te Leiden, Leiden, 1993. MR 1349577 (96e:11114) [3.5]

[225] P. Moree, Nicolaas Govert de Bruijn, the enchanter of friable integers, Indagationes Math., to appear. $[3.5,3.6]$

[226] A. de Morgan, The Differential and Integral Calculus, Baldwin and Craddock: London, 1836-1842. [2.6]

[227] M. Ram Murty and N. Saradha, Transcendental values of the digamma function, J. Number Theory 125 (2007), no. 2, 298-318, DOI 10.1016/j.jnt.2006.09.017. MR.2332591 (2008g:11123) [3.16]

[228] M. Ram Murty and N. Saradha, Euler-Lehmer constants and a conjecture of Erdös, J. Number Theory 130 (2010), no. 12, 2671-2682, DOI 10.1016/j.jnt.2010.07.004. MR2684489 (2011h:11078) [3.8, 3.16]

[229] M. Ram Murty and Anastasia Zaytseva, Transcendence of generalized Euler constants, Amer. Math. Monthly 120 (2013), no. 1, 48-54. MR3007366 [3.16]

[230] V. Kumar Murty, The Euler-Kronecker constant of a cyclotomic field, Ann. Sci. Math. Québec 35 (2011), no. 2, 239-247 (English, with English and French summaries). MR 2917834[4.0]

[231] Yu. V. Nesterenko, Some remarks on ל(3), Mat. Zametki 59 (1996), no. 6, 865-880, 960, DOI 10.1007/BF02307212 (Russian, with Russian summary); English transl., Math. Notes 59 (1996), no. 5-6, 625-636. MR.1445472 (98b:11088)] [3.15]

[232] Yuri V. Nesterenko, Integral identities and constructions of approximations to zeta-values, J. Théor. Nombres Bordeaux 15 (2003), no. 2, 535-550 (English, with English and French summaries). MR2140866 (2006b:11080) [3.15]

[233] Yu. V. Nesterenko, An elementary proof of the irrationality of $\zeta(3)$, Vestnik Moskov. Univ. Ser. I Mat. Mekh. 4 (2009), 28-35, DOI 10.3103/S0027132209040056 (Russian, with English and Russian summaries); English transl., Moscow Univ. Math. Bull. 64 (2009), no. 4, 165171. MR2657274(2011c:11113) [3.15]

[234] I. Newton, Analysis per Quantitatum Series, Fluxiones ac Differentias: cum Enumerationes Lineare Tertii Ordinis, London, 1711. [2.5]

[235] Jürgen Neukirch, Algebraic number theory, With a foreword by G. Harder. Grundlehren der Mathematischen Wissenschaften [Fundamental Principles of Mathematical Sciences], vol. 322, Springer-Verlag, Berlin, 1999. Translated from the 1992 German original and with a note by Norbert Schappacher. MR1697859 (2000m:11104) [4.0]

[236] J.-L. Nicolas, Petites valeurs de la fonction d'Euler et hypothèse de Riemann, Seminar on number theory, Paris 1981-82 (Paris, 1981/1982), Progr. Math., vol. 38, Birkhäuser Boston, Boston, MA, 1983, pp. 207-218 (French). MR729170 (86b:11060) [3.7]

[237] Jean-Louis Nicolas, Petites valeurs de la fonction d'Euler, J. Number Theory 17 (1983), no. 3, 375-388, DOI 10.1016/0022-314X(83)90055-0 (French, with English summary). MR:724536 (85h:11053) [3.7]

[238] Jean-Louis Nicolas, Small values of the Euler function and the Riemann hypothesis, Acta Arith. 155 (2012), no. 3, 311-321, DOI 10.4064/aa155-3-7. MR2983456] [3.1]

[239] Niels Nielsen, Die Gammafunktion. Band I. Handbuch der Theorie der Gammafunktion. Band II. Theorie des Integrallogarithmus und verwandter Transzendenten, Chelsea Publishing Co., New York, 1965 (German). MR0185152 (32 \#2622) [3.1]

[240] Karl K. Norton, Numbers with small prime factors, and the least kth power non-residue, Memoirs of the American Mathematical Society, No. 106, American Mathematical Society, Providence, R.I., 1971. MR0286739 (44 \#3948) [3.5]

[241] V. I. Oseledec, A multiplicative ergodic theorem. Characteristic Ljapunov, exponents of dynamical systems, Trudy Moskov. Mat. Obšč 19 (1968), 179-210 (Russian). MR.0240280 (39 \#1629) [3.13]

[242] D. Panario and B. Richmond, Smallest components in decomposable structures: exp-log class, Algorithmica 29 (2001), no. 1-2, 205-226, DOI 10.1007/BF02679619. Average-case analysis of algorithms (Princeton, NJ, 1998). MR.1887304(2003a:05014) [3.11, 4.0]

[243] B. S. Pavlov and L. D. Faddeev, Scattering theory and automorphic functions, Boundary value problems of mathematical physics and related questions in the theory of functions, 6. Zap. Naučn. Sem. Leningrad. Otdel. Mat. Inst. Steklov (LOMI) 27 (1972), 161-193 (Russian). MR.0320781 (47 \#9315)][3.9] 
[244] D. Pengelley (Translator) Excerpts on the Euler-Maclaurin summation formula, from Institutiones Calculi Differentialis by Leonhard Euler, 28 pages, The Euler Archive http://www. math.dartmouth/ euler/, 2004. [2.4]

[245] David J. Pengelley, Dances between continuous and discrete: Euler's summation formula, Euler at 300, MAA Spectrum, Math. Assoc. America, Washington, DC, 2007, pp. 169-189. MR2349549 (2008j:01004) [2.4]

[246] Friedrich Pillichshammer, Euler's constant and averages of fractional parts, Amer. Math. Monthly 117 (2010), no. 1, 78-83, DOI 10.4169/000298910X475014. MR.2599470 [3.4]

[247] J. M. Pollard, A Monte Carlo method for factorization, Nordisk Tidskr. Informationsbehandling (BIT) 15 (1975), no. 3, 331-334. MR0392798 (52 \#13611) [3.12]

[248] Mark Pollicott, Maximal Lyapunov exponents for random matrix products, Invent. Math. 181 (2010), no. 1, 209-226, DOI 10.1007/s00222-010-0246-y. MR2651384 (2011g:37146) [3.13]

[249] Alfred van der Poorten, A proof that Euler missed ... Apéry's proof of the irrationality of $\zeta(3)$, Math. Intelligencer 1 (1978/79), no. 4, 195-203, DOI 10.1007/BF03028234. An informal report. MR547748 (80i:10054) [3.14]

[250] Marc Prévost, Legendre modified moments for Euler's constant, J. Comput. Appl. Math. 219 (2008), no. 2, 484-492, DOI 10.1016/j.cam.2007.09.015. MR2441241(2009f:11160) [4.0]

[251] P. W. Purdom and J. H. Williams, Cycle length in a random function, Trans. Amer. Math. Soc. 133 (1968), 547-551. MR0228032 (37 \#3616) [3.12]

[252] M. S. Raghunathan, A proof of Oseledec's multiplicative ergodic theorem, Israel J. Math. 32 (1979), no. 4, 356-362, DOI 10.1007/BF02760464. MR571089 (81f:60016) [3.13]

[253] S. Ramanujan, Highly composite numbers, Proc. London Math. Soc. 14 (1915), 347-407. (Also, S. Ramanujan, Collected Papers, Chelsea 1962, pp. 78-129, notes pp. 338-339.) [3.4, 3.7]

[254] S. Ramanujan, A series for Euler's constant $\gamma$, Messenger of Mathematics 46 (1917), 73-80. (Also, S. Ramanujan, Collected Papers, Chelsea 1962, pp. 163-168.) [4.0]

[255] Srinivasa Ramanujan, Highly composite numbers, Ramanujan J. 1 (1997), no. 2, 119-153, DOI 10.1023/A:1009764017495. Annotated and with a foreword by Jean-Louis Nicolas and Guy Robin. MR1606180 (99b:11112) [3.4. 3.7]

[256] p. Rémond de Montmort, Essay D'analyse sur les jeux de hazard, Jacque Quillau, Paris, 1708. [3.11]

[257] P. Rémond de Montmort, Essay d'analyse sur les jeux de hazard, Seconde Edition, Revue $\&$ augmentée de plusieurs Lettres, Jacque Quillau, Paris, 1713. Reprint: American Math. Soc.; Providence 2006. [3.11]

[258] H. J. J. te Riele, "On the history of the function $\frac{M(x)}{\sqrt{x}}$ since Stieltjes", in T. J. Stieltjes, Oeuvres Completes, Volume I, Edited by G. van Dijk, Spriner-Verlag, Berlin, 1993, pp. 6979. $[3.2]$

[259] B. Riemann, Über die Anzahl der Primzahlen unter einer gegebene Grösse, Monatsberichte der Berliner Akademie, Nov. 1859. [3.2]

[260] Tanguy Rivoal, La fonction zêta de Riemann prend une infinité de valeurs irrationnelles aux entiers impairs, C. R. Acad. Sci. Paris Sér. I Math. 331 (2000), no. 4, 267-270, DOI 10.1016/S0764-4442(00)01624-4 (French, with English and French summaries). MR.1787183 (2001k:11138) [3.14]

[261] Tanguy Rivoal, Rational approximations for values of derivatives of the gamma function, Trans. Amer. Math. Soc. 361 (2009), no. 11, 6115-6149, DOI 10.1090/S0002-9947-09-049058. MR2529926 (2011a:11134) [3.15]

[262] Tanguy Rivoal, Approximations rationnelles des valeurs de la fonction gamma aux rationnels, J. Number Theory 130 (2010), no. 4, 944-955, DOI 10.1016/j.jnt.2009.08.003 (French, with English summary). MR2600413 (2011i:11109) [3.15]

[263] Tanguy Rivoal, Approximations rationnelles des valeurs de la fonction gamma aux rationnels: le cas des puissances, Acta Arith. 142 (2010), no. 4, 347-365, DOI 10.4064/aa1424-4 (French). MR 2640064(2012c:11150) [3.15]

[264] T. Rivoal, On the arithmetic nature of the values of the gamma function, Euler's constant, and Gompertz's constant, Michigan Math. J. 61 (2012), no. 2, 239-254, DOI $10.1307 / \mathrm{mmj} / 1339011525 . \operatorname{MR} 2944478[2.5,3.16]$ 
[265] G. Robin, Grandes valeurs de la fonction somme des diviseurs et hypothèse de Riemann, J. Math. Pures Appl. (9) 63 (1984), no. 2, 187-213 (French, with English summary). MR.774171 (86f:11069) [3.7]

[266] E. Sandifer, The Early Mathematics of Leonhard Euler. MAA, Washington, DC 2007. [2.0]

[267] E. Sandifer, Euler's solution of the Basel problem - the longer story, in: Euler at 300, MAA Spectrum, pp. 105-117, Math Assoc. of America, Washington, DC, 2007. [2.0]

[268] L. A. Shepp and S. P. Lloyd, Ordered cycle lengths in a random permutation, Trans. Amer. Math. Soc. 121 (1966), 340-357. MR0195117 (33 \#3320) [3.10, 3.12]

[269] A. B. Šidlovskiǔ, Transcendence and algebraic independence of the values of entire functions of certain classes, Moskov. Gos. Univ. Uč. Zap. No. 186 (1959), 11-70 (Russian). MR 0136579 (25 \#49) [3.16]

[270] A. B. Sidlovskiı̌, Transcendency and algebraic independence of values of E-functions related by an arbitrary number of algebraic equations over the field of rational functions, Izv. Akad. Nauk SSSR Ser. Mat. 26 (1962), 877-910 (Russian). MR0144854 (26 \#2395)] [3.16]

[271] Andrei Borisovich Shidlovskii, Transcendental numbers, Translated from the Russian by Neal Koblitz; With a foreword by W. Dale Brownawell, de Gruyter Studies in Mathematics, vol. 12, Walter de Gruyter \& Co., Berlin, 1989. MR.1033015 (90j:11066) [3.16]

[272] C. L. Siegel, Über einige Andwendungen diophantischer Approximationen, Abh. Preuss. Akad. Wiss., Phys.-Math. Kl. 1 (1929), 1-70. (Carl Ludwig Siegel, Gesammelte Abhandlunden, Vol. I, Springer-Verlag, Berlin, 1966, pp. 209-266.) [3.16]

[273] Carl Ludwig Siegel, Transcendental Numbers, Annals of Mathematics Studies, no. 16, Princeton University Press, Princeton, N. J., 1949. MR0032684 (11,330c) [3.16]

[274] N. J. A. Sloane, The On-Line Encyclopedia of Integer Sequences, http://oeis/org. [3.15]

[275] J. G. von Soldner, Théorie et tables d'une nouvelle fonction transcendante, Lindauer, München, 1809. [2.6]

[276] Jonathan Sondow, Criteria for irrationality of Euler's constant, Proc. Amer. Math. Soc. 131 (2003), no. 11, 3335-3344, DOI 10.1090/S0002-9939-03-07081-3. MR1990621 (2004b:11102) [3.15]

[277] Jonathan Sondow, Double integrals for Euler's constant and $\ln \frac{4}{\pi}$ and an analog of Hadjicostas's formula, Amer. Math. Monthly 112 (2005), no. 1, 61-65, DOI 10.2307/30037385. MR2110113(2005i:11181) [4.0]

[278] Jonathan Sondow, A hypergeometric approach, via linear forms involving logarithms, to criteria for irrationality of Euler's constant, With an appendix by Sergey Zlobin, Math. Slovaca 59 (2009), no. 3, 307-314, DOI 10.2478/s12175-009-0127-2. MR2505810(2010i:11108) [3.15]

[279] Jonathan Sondow, New Vacca-type rational series for Euler's constant $\gamma$ and its "alternating" analog $\ln \frac{4}{\pi}$, Additive Number Theory, Springer, New York, 2010, pp. 331-340, DOI 10.1007/978-0-387-68361-4_23. MR2744766 (2012b:11202) [3.15]

[280] Jonathan Sondow and Petros Hadjicostas, The generalized-Euler-constant function $\gamma(z)$ and a generalization of Somos's quadratic recurrence constant, J. Math. Anal. Appl. 332 (2007), no. 1, 292-314, DOI 10.1016/j.jmaa.2006.09.081. MR.2319662(2008f:40013) [4.0]

[281] J. Sondow and S. A. Zlobin, Integrals over polytopes, multiple zeta values and polylogarithms, and Euler's constant, Mat. Zametki 84 (2008), 609-626. [3.15]

[282] Jonathan Sondow and Wadim Zudilin, Euler's constant, q-logarithms, and formulas of Ramanujan and Gosper, Ramanujan J. 12 (2006), no. 2, 225-244, DOI 10.1007/s11139-0060075-1. MR2286247(2007j:11182) [3.15]

[283] K. Soundararajan, Omega results for the divisor and circle problems, Int. Math. Res. Not. 36 (2003), 1987-1998, DOI 10.1155/S1073792803130309. MR1991181 (2004f:11105) [3.4]

[284] K. Soundararajan, An asymptotic expansion related to the Dickman function, Ramanujan J. 29 (2012), no. 1-3, 25-30, DOI 10.1007/s11139-011-9304-3. MR2994087 [4.0]

[285] P. Stäckel, Eine vergessene Abhandlung Leonhard Eulers über die Summe der reziproken Quadrate der natürlichen Zahlen, Bibliotheca Mathematica, 3rd series 8 (1907/08), 37-60. $[2.0]$

[286] T.J. Stieltjes, Sur un fonction uniforme, C. R. Acad. Sci. Paris. 101, 153-154, 13 Juillet 1885. [In: Oeuvres Compétes, Volume I, Paper XLIV. English Translation: "On a uniform function," p. 561.] [3.2]

[287] T.-J. Stieltjes, Recherches sur quelques séries semi-convergentes, Ann. Sci. École Norm. Sup. (3) 3 (1886), 201-258 (French). MR 1508781 [3.1] 
[288] T. J. Stieltjes, Sur le dévellopment de $\log \Gamma(a)$, J. Math. Pures Appl. (4) 5 (1889), 425-444. (In: Oeuvres Complétes, Volume II, Paper LXX). [3.1]

[289] T.-J. Stieltjes, Recherches sur les fractions continues, Ann. Fac. Sci. Toulouse Sci. Math. Sci. Phys. 8 (1894), no. 4, J1-J122 (French). MR1508159 [2.5]

[290] T. J. Stieltjes, Oeuvres Completes, Volumes I, II, Edited by G. van Dijk, Springer-Verlag: Berlin 1993. [3.2]

[291] J. Stirling, Methodus differentialis sive tractatus de summatione et interpolatione serierum infinitarum, Whiston and White, London, 1730. [English Translation: [Twe03]] [2.5]

[292] Dura W. Sweeney, On the computation of Euler's constant, Math. Comp. 17 (1963), 170178. MR0160308(28 \#3522) [3.15]

[293] Gérald Tenenbaum, Introduction to analytic and probabilistic number theory, Translated from the second French edition (1995) by C. B. Thomas, Cambridge Studies in Advanced Mathematics, vol. 46, Cambridge University Press, Cambridge, 1995. MR 1342300 $(97 \mathrm{e}: 11005 \mathrm{~b})[2.2,3.5,3.6]$

[294] Gérald Tenenbaum and Michel Mendès France, The prime numbers and their distribution, Translated from the 1997 French original by Philip G. Spain, Student Mathematical Library, vol. 6, American Mathematical Society, Providence, RI, 2000. MR.1756233 (2001j:11086) $[3.3]$

[295] Tomohide Terasoma, Confluent hypergeometric functions and wild ramification, J. Algebra 185 (1996), no. 1, 1-18, DOI 10.1006/jabr.1996.0309. MR1409971 (98k:11080) [3.14]

[296] Tomohide Terasoma, Selberg integrals and multiple zeta values, Compositio Math. 133 (2002), no. 1, 1-24, DOI 10.1023/A:1016377828316. MR1918286(2003k:11142) [3.14]

[297] Tomohide Terasoma, Mixed Tate motives and multiple zeta values, Invent. Math. 149 (2002), no. 2, 339-369, DOI 10.1007/s002220200218. MR.1918675(2003h:11073)] [3.14]

[298] Tomohide Terasoma, Geometry of multiple zeta values, International Congress of Mathematicians. Vol. II, Eur. Math. Soc., Zürich, 2006, pp. 627-635. MR2275614 (2008e:14009) [3.14]

[299] E. C. Titchmarsh, On the function $\frac{1}{|\zeta(1+i t)|}$, Quarterly J. Math. (Oxford) 4 (1933), 60-70. $[3.9]$

[300] E. C. Titchmarsh, The theory of the Riemann zeta-function, 2nd ed., Edited and with a preface by D. R. Heath-Brown. The Clarendon Press Oxford University Press, New York, 1986. MR882550 (88c:11049) [3.9]

[301] Jacques Touchard, Sur les cycles des substitutions, Acta Math. 70 (1939), no. 1, 243-297, DOI 10.1007/BF02547349 (French). MR1555449 [3.10]

[302] Kai-Man Tsang, Recent progress on the Dirichlet divisor problem and the mean square of the Riemann zeta-function, Sci. China Math. 53 (2010), no. 9, 2561-2572, DOI 10.1007/s11425010-4068-6. MR2718848(2011m:11196) [3.4]

[303] C. Truesdell, An idiot's fugitive essays on science, Methods, criticism, training, circumstances. Springer-Verlag, New York, 1984. MR769106 (86g:01060) [2.6]

[304] C. Truesdell, Great scientists of old as heretics in "the scientific method", University Press of Virginia, Charlottesville, VA, 1987. MR915762 (88m:01038) [2.6]

[305] D. N. Tulyakov, A system of recurrence relations for rational approximations of the Euler constant, Mat. Zametki 85 (2009), no. 5, 782-787, DOI 10.1134/S0001434609050150 (Russian, with Russian summary); English transl., Math. Notes 85 (2009), no. 5-6, 746750. MR2572868(2011d:11029) [3.15]

[306] I. Tweddle, James Stirling "This about series and such things", Scottish Academic Press, Edinburgh, 1988. [2.5]

[307] Ian Tweddle, James Stirling's early work on acceleration of convergence, Arch. Hist. Exact Sci. 45 (1992), no. 2, 105-125, DOI 10.1007/BF00374250. MR1201631 (94d:01020) [2.5]

[308] I. Tweddle, James Stirling's Methodus Differentialis. An Annotated Translation of Stirling's Text. Springer: New York 2003. [2.5]

[309] G. Vacca, A new series for the Eulerian constant, Quart. J. Pure Appl. Math. (Oxford) 41 (1910), 363-368. [3.15]

[310] V. S. Varadarajan, Euler through time: a new look at old themes, American Mathematical Society, Providence, RI, 2006. MR2219954 (2007h:01009) [2.0]

[311] A. M. Vershik and A. A. Shmidt, Limit measures arising in the asymptotic theory of symmetric groups, II, Theor. Prob. Appl. 23 (1978), no. 1, 36-49. MR0483019 (58\#3049)[3.11] 
[312] Michel Waldschmidt, Transcendence of periods: the state of the art, Pure Appl. Math. Q. 2 (2006), no. 2, 435-463. MR2251476(2007d:11083)] [3.14]

[313] H. S. Wall, Analytic Theory of Continued Fractions, D. Van Nostrand Company, Inc., New York, N. Y., 1948. MR0025596(10,32d) [2.5]

[314] J. Wallis [Johannes Wallis], Arithmetica Infinitorum, sive Nova Methodus Inquirendi in Curvilineorum Quadraturam; aliaq; difficiliora Matheseos Problemata. [Arithmetic of Infinities], Oxon: Tho. Robinson, Lichfield 1656. [2.5]

[315] A. Weil, Number Theory: An approach through history from Hammurapi to Legendre, Birkhäuser-Boston, Inc., Boston, 1984. [2.0, 2.4]

[316] Ferrell S. Wheeler, Two differential-difference equations arising in number theory, Trans. Amer. Math. Soc. 318 (1990), no. 2, 491-523, DOI 10.2307/2001317. MR.963247|(90g:11134) $[3.6,3.10]$

[317] E. T. Whittaker and J. M. Watson, A Course of Modern Analysis. Fourth Edition Cambridge Univ. Press, Cambridge 1963. [2.2]

[318] J. W. Wrench, Jr. A new calculation of Euler's constant, Mathematical Tables and Aids to Computation (MTAC) 6 (1952), 255. [3.15]

[319] Don Zagier, A Kronecker limit formula for real quadratic fields, Math. Ann. 213 (1975), 153-184. MR0366877 (51 \#3123) [4.0]

[320] Don Zagier, Values of zeta functions and their applications, First European Congress of Mathematics, Vol. II (Paris, 1992), Progr. Math., vol. 120, Birkhäuser, Basel, 1994, pp. 497512. MR1341859 (96k:11110) [3.14]

[321] V. V. Zudilin, One of the numbers $\zeta(5), \zeta(7), \zeta(9), \zeta(11)$ is irrational, Uspekhi Mat. Nauk 56 (2001), no. 4(340), 149-150, DOI 10.1070/RM2001v056n04ABEH000427 (Russian); English transl., Russian Math. Surveys 56 (2001), no. 4, 774-776. MR1861452 (2002g:11098) [3.14]

Department of Mathematics, University of Michigan, Ann Arbor, Michigan 481091043

E-mail address: lagarias@umich.edu 